\title{
The chemical make-up of the Sun: A 2020 vision
}

\author{
M. Asplund ${ }^{1} \odot$, A. M. Amarsi ${ }^{2} \odot$, and N. Grevesse $e^{3,4}$ \\ 1 Australian Academy of Science, Box 783, Canberra ACT 2601, Australia \\ e-mail: martin.b.asplund@gmail.com \\ 2 Theoretical Astrophysics, Department of Physics and Astronomy, Uppsala University, Box 516, 75120 Uppsala, Sweden \\ 3 Centre Spatial de Liège, Université de Liège, avenue Pré Aily, 4031 Angleur-Liège, Belgium \\ ${ }^{4}$ Space Sciences, Technologies and Astrophysics Research (STAR) Institute, Université de Liège, Allée du 6 août, 17, B5C, \\ 4000 Liège, Belgium
}

Received 28 January 2021 / Accepted 3 May 2021

\begin{abstract}
Context. The chemical composition of the Sun is a fundamental yardstick in astronomy, relative to which essentially all cosmic objects are referenced. As such, having accurate knowledge of the solar elemental abundances is crucial for an extremely broad range of topics.

Aims. We reassess the solar abundances of all 83 long-lived elements, using highly realistic solar modelling and state-of-the-art spectroscopic analysis techniques coupled with the best available atomic data and observations.

Methods. The basis for our solar spectroscopic analysis is a three-dimensional (3D) radiative-hydrodynamical model of the solar surface convection and atmosphere, which reproduces the full arsenal of key observational diagnostics. New complete and comprehensive 3D spectral line formation calculations taking into account of departures from local thermodynamic equilibrium (non-LTE) are presented for $\mathrm{Na}, \mathrm{Mg}, \mathrm{K}, \mathrm{Ca}$, and $\mathrm{Fe}$ using comprehensive model atoms with reliable radiative and collisional data. Our newly derived abundances for $\mathrm{C}, \mathrm{N}$, and $\mathrm{O}$ are based on a 3D non-LTE analysis of permitted and forbidden atomic lines as well as 3D LTE calculations for a total of 879 molecular transitions of $\mathrm{CH}, \mathrm{C}_{2}, \mathrm{CO}, \mathrm{NH}, \mathrm{CN}$, and $\mathrm{OH}$. Previous 3D-based calculations for another 50 elements are re-evaluated based on updated atomic data, a stringent selection of lines, improved consideration of blends, and new non-LTE calculations available in the literature. For elements where spectroscopic determinations of the quiet Sun are not possible, the recommended solar abundances are revisited based on complementary methods, including helioseismology (He), solar wind data from the Genesis sample return mission (noble gases), sunspot observations (four elements), and measurements of the most primitive meteorites (15 elements).

Results. Our new improved analysis confirms the relatively low solar abundances of $\mathrm{C}, \mathrm{N}$, and $\mathrm{O}$ obtained in our previous 3D-based studies: $\log \epsilon_{\mathrm{C}}=8.46 \pm 0.04, \log \epsilon_{\mathrm{N}}=7.83 \pm 0.07$, and $\log \epsilon_{\mathrm{O}}=8.69 \pm 0.04$. Excellent agreement between all available atomic and molecular indicators is achieved for $\mathrm{C}$ and $\mathrm{O}$, but for $\mathrm{N}$ the atomic lines imply a lower abundance than for the molecular transitions for unknown reasons. The revised solar abundances for the other elements also typically agree well with our previously recommended values, with only $\mathrm{Li}, \mathrm{F}, \mathrm{Ne}, \mathrm{Mg}, \mathrm{Cl}, \mathrm{Kr}, \mathrm{Rb}, \mathrm{Rh}, \mathrm{Ba}, \mathrm{W}$, Ir, and $\mathrm{Pb}$ differing by more than 0.05 dex. The here-advocated present-day photospheric metal mass fraction is only slightly higher than our previous value, mainly due to the revised Ne abundance from Genesis solar wind measurements: $X_{\text {surface }}=0.7438 \pm 0.0054, Y_{\text {surface }}=0.2423 \pm 0.0054, Z_{\text {surface }}=0.0139 \pm 0.0006$, and $Z_{\text {surface }} / X_{\text {surface }}=0.0187 \pm 0.0009$. Overall, the solar abundances agree well with those of CI chondritic meteorites, but we identify a correlation with condensation temperature such that moderately volatile elements are enhanced by $\approx 0.04$ dex in the CI chondrites and refractory elements possibly depleted by $\approx 0.02 \mathrm{dex}$, conflicting with conventional wisdom of the past half-century. Instead, the solar chemical composition more closely resembles that of the fine-grained matrix of CM chondrites with the expected exception of the highly volatile elements.

Conclusions. Updated present-day solar photospheric and proto-solar abundances are presented for 83 elements, including for all long-lived isotopes. The so-called solar modelling problem - a persistent discrepancy between helioseismology and solar interior models constructed with a low solar metallicity similar to that advocated here - remains intact with our revised solar abundances, suggesting shortcomings with the computed opacities and/or treatment of mixing below the convection zone in existing standard solar models. The uncovered trend between the solar and CI chondritic abundances with condensation temperature is not yet understood but is likely imprinted by planet formation, especially since a similar trend of opposite sign is observed between the Sun and solar twins.
\end{abstract}

Key words. Sun: abundances - Sun: photosphere - Sun: atmosphere - line: formation - meteorites, meteors, meteoroids Sun: helioseismology

\section{Introduction}

The pursuit of detailed knowledge of the elemental abundances in the Solar System has played a critical role in the development of modern astronomy. Since the chemical make-up of the planets is not expected to precisely reflect the composition of the nebula from which the Sun and planets formed, there are two methods to determine the proto-solar abundances, both of which were initiated a century ago: solar spectroscopy (Payne 1925; Russell 1929) and mass spectroscopy of primitive meteorites (Goldschmidt 1922, 1938). The two techniques each have their advantages and are thus highly complementary. Indeed a common approach is to combine the meteoritic and photospheric measurements to estimate the Solar System's composition, starting from the early influential works by Unsöld (1948) and Suess \& Urey (1956), who provided 
a large amount of abundance data for the seminal papers on the origin of the elements by Burbidge et al. (1957) and Cameron (1957). More recently, the widely used literature compilations by Anders \& Grevesse (1989), Grevesse et al. (1993), Grevesse \& Sauval (1998), Lodders (2003, 2019), Lodders et al. (2009), and Palme et al. (2014) adopted this approach with a preference for the meteoritic values for most elements on account of their typically higher precision ${ }^{1}$. Due to severe depletion of the highly volatile elements $-\mathrm{H}, \mathrm{He}, \mathrm{C}, \mathrm{N}, \mathrm{O}, \mathrm{Ne}, \mathrm{Ar}, \mathrm{Xe}$, and $\mathrm{Kr}$ - meteorites cannot, however, be relied upon to measure the bulk Solar System composition, for which the Sun is indispensable. There is also no guarantee that the abundances of other elements in the most primitive meteorites, the CI chondrites, truly reflect those of the Sun; indeed, this long-held assumption is not correct, as we show here.

The photospheric abundances are certainly not without shortcomings themselves. While often referred to as observed elemental abundances, the chemical composition of the Sun cannot be directly measured from the observed solar spectrum. Inferring the chemical make-up of a star requires a realistic model for the stellar atmosphere and of the transfer of radiation coupled with all atomic processes that shape the predicted emergent radiation to be compared with the observed spectrum. By necessity, the derived solar (and stellar) abundances are model dependent and therefore are not more reliable than the models from which they are inferred. Traditionally, quantitative solar spectroscopy has been done by means of one-dimensional (1D) model atmospheres: either theoretical models in radiative and convective equilibrium (e.g., Kurucz 1993; Gustafsson et al. 2008) or semi-empirical models in which the atmospheric temperature stratification is deduced from observations of the continuum centre-to-limb variation and of line depths, for example (e.g., Holweger \& Müller 1974; Allende Prieto et al. 2001a; Fontenla et al. 2006). Neither type of 1D model attempts to describe the temporal variations or horizontal inhomogeneities in the atmosphere, and as such they include at best only a rudimentary description of convective energy transport (e.g., mixing length theory, Böhm-Vitense 1958).

A huge body of influential and careful work on determining the solar chemical composition has been carried out over several decades using such 1D solar atmosphere models (e.g., Goldberg et al. 1960; Lambert 1968, 1978; Grevesse \& Sauval 1973, 1999; Ross \& Aller 1976; Holweger et al. 1991; Blackwell et al. 1995). This has often been done in close collaboration with atomic physicists measuring or computing transition probabilities and other necessary input data, which is a highly successful synergy (e.g., Garz et al. 1969; Andersen et al. 1975; Biemont et al. 1981, 1991; Hannaford et al. 1992; Barklem et al. 2000; Lawler et al. 2001, 2013; Johansson et al. 2003; Ljung et al. 2006; Blackwell-Whitehead \& Bergemann 2007; Den Hartog et al. 2019). These abundance analyses have mostly been within the framework of local thermodynamic equilibrium (LTE), thus assuming the Boltzmann, Saha, and Guldberg-Waage distributions for the atomic and molecular level populations and the Planck function for the radiation source function (e.g., Rutten 2003). While LTE is for many spectral lines that form in the solar photosphere a reasonable approximation, its validity cannot be taken for granted. For

\footnotetext{
1 An indication of the incredible impact the solar elemental abundances have across astronomy and beyond is the fact that Anders \& Grevesse (1989) is among the ten most cited astronomy articles of all time with more than 8000 citations to date according to ADS, or more than 12000 citations in Google Scholar.
}

some elements, departures from LTE for the line formation in 1D model atmospheres (here: 1D non-LTE) and their impact on the inferred solar abundances have been explored (e.g., Steenbock \& Holweger 1984; Kiselman 1991; Takeda 1994; Korn et al. 2003; Mashonkina et al. 2011; Lind et al. 2011; Bergemann et al. 2012; Shi et al. 2014). Unfortunately, such statistical equilibrium calculations have still not been performed for the majority of the elements, rendering their solar abundances prone to systematic errors (Asplund 2005; Bergemann et al. 2012; Nissen \& Gustafsson 2018).

With increased computational power, it has become possible to perform 3D (magneto-)hydrodynamical simulations of the outer convective and atmospheric layers in the Sun and other stars (e.g., Nordlund \& Dravins 1990; Freytag et al. 1996; Ludwig et al. 1999; Asplund et al. 1999; Vögler et al. 2005; Collet et al. 2007; Muthsam et al. 2010; Magic et al. 2013; Trampedach et al. 2013; Beeck et al. 2013). In such modelling, the equations for the conservation of mass, momentum, and energy are solved together with the radiative transfer equation in a $3 \mathrm{D}$ simulation box covering a small but representative volume of the star. Besides shedding crucial light on, for example, the nature of convective energy transport (e.g., Stein \& Nordlund 1998), the excitation and damping of solar and stellar oscillations (e.g., Sonoi et al. 2015; Zhou et al. 2019), and the dynamo-generation of stellar magnetic fields (e.g., Vögler \& Schüssler 2007; Rempel 2014), such 3D simulations are eminently suited as model atmospheres for the prediction of the emergent radiation spectrum and thus for solar abundance analyses (e.g., Kiselman \& Nordlund 1995; Asplund et al. 2000c, 2005b; Allende Prieto et al. 2001b; Holweger 2001; Caffau et al. 2008, 2011). Because convection emerges naturally in a self-consistent manner, there is no need to invoke the mixing length theory. These $3 \mathrm{D}$ hydrodynamical models are parameter-free in the sense that there is no tuning of any parameters involved to improve agreement with observations, yet their granulation properties, spectral line shapes, continuum centre-to-limb variations, and spectral energy distributions agree extremely well with observations; the choice of, for example, numerical resolution and sub-grid treatment does not impact the predictions (e.g., Asplund et al. 2000a; Nordlund et al. 2009; Pereira et al. 2013). Importantly, this is achieved without invoking any micro- and macro-turbulence parameters since the convective motions and stellar oscillations introduce the necessary Doppler shifts together with the (anti-)correlations in temperature and velocities in granules and intergranular lanes to explain the line strengths, widths, shifts and asymmetries (Asplund et al. 2000b). Clearly, the most recent 3D hydrodynamical solar models, such as the one employed herein, are highly realistic and far superior to any $1 \mathrm{D}$ model.

Today it is also feasible to carry out 3D non-LTE line formation calculations with comprehensive model atoms involving the relevant radiative and collisional processes for solar/stellar abundance purposes (e.g., Asplund et al. 2003, 2004; Cayrel et al. 2007; Lind et al. 2013; Steffen et al. 2015; Amarsi et al. 2016a, 2019; Bergemann et al. 2019). Such statistical equilibrium computations, while computationally demanding ${ }^{2}$, make optimal use

\footnotetext{
2 The 3D non-LTE calculations are dominated by the formal solution of the radiative transfer equation. While in 1D LTE the synthesis of a single spectral line requires radiative transfer solutions along a ray for $\sim 100$ frequencies and a handful of angles; in 3D non-LTE, this typically has to be done for $\sim 10^{4}$ frequencies involving all populated atomic levels and transitions, $\sim 25$ angles, $\sim 10^{4}$ atmospheric columns, and 20-50 iterations to converge the rate equations for statistical equilibrium and for $\sim 5$ temporal snapshots, that is, $>10^{9}$ equivalent 1D LTE calculations.
} 
of the highly realistic 3D hydrodynamical stellar surface convection models and self-consistently describe departures from the LTE within the trace-element assumption. The non-LTE effects can typically be expected to be exacerbated in 3D compared to 1D modelling (Asplund 2005). The field has benefited tremendously from the increased availability of accurate atomic data for the vast number of radiative and collisional cross-sections required, including the previously highly uncertain inelastic collisions with electrons and with neutral hydrogen, which are of paramount importance in the atmospheres of late-type stars (e.g., Barklem 2016a). It is truly remarkable that all free parameters - mixing length parameters, microturbulence, macroturbulence, Unsöld enhancement factor for pressure broadening, Drawin scaling factor for $\mathrm{H}$ collisions, etc - which have hampered quantitative stellar spectroscopy for decades, are now finally obsolete. Together with vastly improved stellar modelling and transition probabilities, this has drastically improved the accuracy and precision of the inferred stellar chemical compositions.

Asplund et al. (2009) presented the first fully 3D-based analysis of the solar photospheric elemental abundances with nonLTE calculations (usually based on 1D model atmospheres) for the most important elements; earlier 3D determinations for many elements were reported in for example Asplund et al. (2000c, 2004, 2005a,b). It was also the first time all spectroscopically available elements were analysed in a homogeneous manner and with the systematic uncertainties quantified in detail (see also Scott et al. 2015a,b and Grevesse et al. 2015 for further details).

Here, we discuss further improvements to our solar analysis, including new 3D non-LTE calculations for many more elements, better atomic data, and refined line selection, which have led to a revision of the abundances of numerous elements. We argue that our study represents a state-of-the-art determination of the solar chemical composition, yielding the most reliable results available today. Complemented with precise laboratory measurements of the compositions of the most pristine meteorites (e.g., Palme et al. 2014), the new solar abundances enable us to uncover subtle abundance differences with condensation temperatures of the elements between the Sun and CI chondrites, which reflect the complicated processes of star and planet formation. For convenience, we also provide estimates for the proto-solar (isotopic) abundances where the effects of atomic diffusion, nuclear burning, and radioactive decay have been taken into account, as well as the present-day and protosolar mass fractions of $\mathrm{H}, \mathrm{He}$, and heavy elements.

\section{Present-day photospheric solar abundances}

Table 1 intercompares the main ingredients of this study and our previous 3D-based solar abundance analyses in terms of 3D solar modelling and spectral line formation, showing that most recent progress has concerned non-LTE calculations, as described further below. The recommended solar photospheric elemental abundances presented in Table 2 and shown in Fig. 1 represent, in our opinion, the most reliable measurements available today, obtained with state-of-the-art analysis techniques and the best atomic and molecular data. All abundances have wellquantified and justified uncertainties and supersede those given in our previous summaries of the solar chemical composition (e.g., Asplund et al. 2005a, 2009).

The abundances are expressed on the traditional astronomical logarithmic abundance scale. Hydrogen is the natural reference element for solar (and stellar) spectroscopy, both because it is the most abundant element and because it provides the continuous opacity in the optical and infrared through the negative

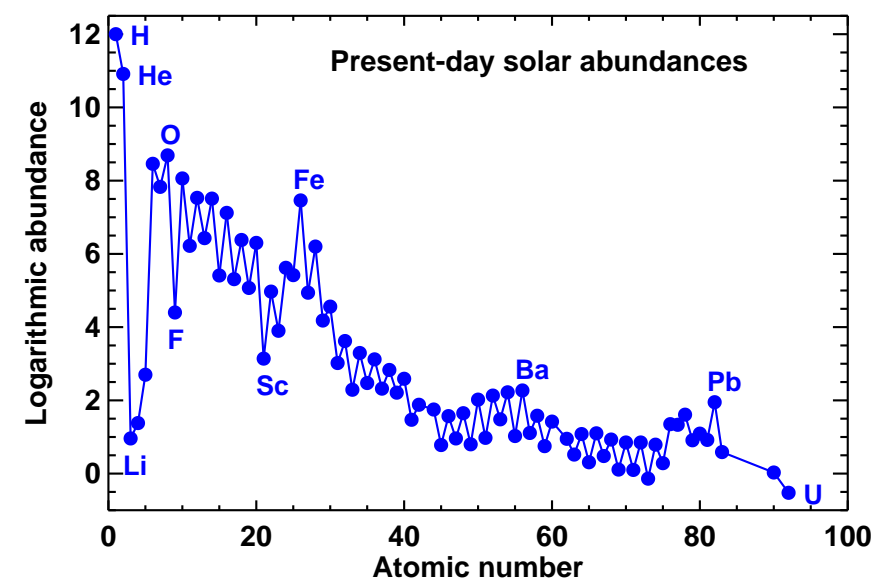

Fig. 1. Present-day solar photospheric logarithmic abundances (by number) as a function of atomic number, with $\mathrm{H}$ defined as $\log \epsilon_{\mathrm{H}} \equiv 12.00$. For elements for which no photospheric determination has been possible the CI chondritic abundance is shown, corrected for the identified correlation with condensation temperature (Sect. 3.3). $\mathrm{H}$ and $\mathrm{He}$ are the most abundant elements on account of being produced in the Big Bang with some contribution to He from stellar H-burning. Prominent peaks occur around $\mathrm{O}, \mathrm{Fe}, \mathrm{Ba}$, and $\mathrm{Pb}$, while elements with an even atomic number have higher abundances than neighbouring odd elements (so-called odd-even effect) as a result of stellar evolution and nuclear physics (minimum nuclear binding energy and nuclei magic numbers). $\mathrm{Li}, \mathrm{F}$, and $\mathrm{Sc}$ have particularly low abundances relative to nearby elements on account of them being odd elements, having relatively low binding energy, and not being part of the main nucleosynthesis production channels in stars. We note that the unstable elements Tc $(Z=43)$ and $\operatorname{Pm}(Z=61)$ are not shown.

hydrogen ion $\mathrm{H}^{-}$. The normalisation of the elemental number density $N_{\mathrm{X}}$ for an element $\mathrm{X}$ is defined as $\log \epsilon_{\mathrm{X}} \equiv \log \left(N_{\mathrm{X}} / N_{\mathrm{H}}\right)+$ 12.00 , for historical reasons, hence $\log \epsilon_{\mathrm{H}} \equiv 12.00^{3}$.

After first summarising some central ingredients in our analysis, in the following sub-sections we present a detailed discussion of the elements for which solar photospheric abundances have been newly analysed, compared with our previous spectroscopic studies of elements heavier than $\mathrm{Ne}$ (Scott et al. 2015a,b; Grevesse et al. 2015). In some cases, we simply updated their results in light of new atomic data (in particular, transition probabilities) or improved non-LTE abundance corrections, or we made a different choice in terms of the line list or weighting of the individual lines. For $\mathrm{Li}, \mathrm{C}, \mathrm{N}, \mathrm{O}, \mathrm{Na}, \mathrm{Mg}, \mathrm{Al}, \mathrm{Si}, \mathrm{K}, \mathrm{Ca}$, $\mathrm{Fe}$, and $\mathrm{Ba}$, our results are based on new 3D non-LTE calculations. We also discuss the elements that are not accessible by spectroscopy of the quiet Sun for which other methods to infer the solar abundances are required.

\subsection{General considerations of the analysis}

Model atmospheres. As a default solar model atmosphere, we employed the same 3D hydrodynamic simulation of the solar surface convection computed with the Stagger code (Nordlund \& Galsgaard 1995; Stein \& Nordlund 1998; Magic et al. 2013), as was done in our recent studies of 3D

3 This choice was supposedly to avoid having negative elemental abundances for the Sun (Claas 1951; Goldberg et al. 1960). While this is also true for the here recommended solar photospheric abundances - barely, with $\log \epsilon_{\mathrm{Th}}=0.03 \pm 0.10$ being the smallest value - several naturally occurring elements indeed have $\log \epsilon<0$ in CI chondritic meteorites (Sect. 3): Ne, Ar, Kr, Xe, Ta, and U. 
Table 1. Summary of main ingredients of our 3D-based solar abundance analyses.

\begin{tabular}{|c|c|c|c|}
\hline Ingredient & Asplund et al. (2005a) & Asplund et al. (2009) & This work \\
\hline \multicolumn{4}{|l|}{ 3D model atmosphere: } \\
\hline Code & Convec & Stagger & Stagger \\
\hline Code reference & Stein \& Nordlund (1998) & Nordlund \& Galsgaard (1995) & Magic et al. (2013) \\
\hline Input abundances & $\begin{array}{c}\text { Anders \& Grevesse (1989), } \\
\log \epsilon_{\mathrm{He}}=10.92, \log \epsilon_{\mathrm{Fe}}=7.50\end{array}$ & Asplund et al. (2005a) & Asplund et al. (2009) \\
\hline Equation of state & Mihalas et al. (1988) & Trampedach et al. (2013) & Trampedach et al. (2013) \\
\hline Continuous opacities & Gustafsson et al. (1975) & Gustafsson et al. (2008) & Hayek et al. (2010) \\
\hline Line opacities & Kurucz (1993) & Gustafsson et al. (2008) & Gustafsson et al. (2008) \\
\hline Numerical resolution & $200^{2} \times 82$ & $240^{3}$ & $240^{3}$ \\
\hline Geometrical dimension & $6 \times 6 \times 3.5 \mathrm{Mm}$ & $6 \times 6 \times 3.5 \mathrm{Mm}$ & $6 \times 6 \times 3.5 \mathrm{Mm}$ \\
\hline Opacity bins & 4 (opacity) & 12 (opacity \& wavelength) & 12 (opacity \& wavelength) \\
\hline Effective temperature $T_{\text {eff }} \pm$ s.d. & $5767 \pm 21 \mathrm{~K}$ & $5778 \pm 5 \mathrm{~K}$ & $5773 \pm 16 \mathrm{~K}$ \\
\hline Continuum CLV & too steep $T(\tau)$ & good agreement w/obs. & good agreement w/obs. \\
\hline \multicolumn{4}{|l|}{ Spectral line formation: } \\
\hline Full 3D non-LTE & $\mathrm{Li}, \mathrm{O}$ & $\mathrm{Li}, \mathrm{O}$ & $\begin{array}{c}\mathrm{Li}, \mathrm{C}, \mathrm{N}, \mathrm{O}, \mathrm{Na}, \mathrm{Mg}, \mathrm{Al} \\
\text { Si, K, Ca, Fe, Ba }\end{array}$ \\
\hline 3D LTE + 1D/〈3D $\rangle$ non-LTE & $\mathrm{C}, \mathrm{N}, \mathrm{Na}, \mathrm{Mg}, \mathrm{Al}, \mathrm{K}$ & $\begin{array}{l}\mathrm{C}, \mathrm{N}, \mathrm{Na}, \mathrm{Mg}, \mathrm{Al}, \mathrm{Si}, \mathrm{S}, \mathrm{K} \\
\mathrm{Ca}, \mathrm{Sc}, \mathrm{Ti}, \mathrm{Cr}, \mathrm{Mn}, \mathrm{Fe}, \mathrm{Co} \\
\mathrm{Cu}, \mathrm{Zn}, \mathrm{Sr}, \mathrm{Ba}, \mathrm{Eu}, \mathrm{Pb}, \mathrm{Th}\end{array}$ & $\begin{array}{l}\mathrm{S}, \mathrm{Sc}, \mathrm{Ti}, \mathrm{Cr}, \mathrm{Mn}, \mathrm{Co}, \mathrm{Cu} \\
\mathrm{Zn}, \mathrm{Rb}, \mathrm{Sr}, \mathrm{Pr}, \mathrm{Eu}, \mathrm{Pb}, \mathrm{Th}\end{array}$ \\
\hline $\begin{array}{l}\text { 3D LTE } \\
\text { 1D ITE (literature) }\end{array}$ & $\mathrm{Be}, \mathrm{B}, \mathrm{Si}, \mathrm{P}, \mathrm{S}, \mathrm{Ca}, \mathrm{Fe}$ & $\mathrm{Be}, \mathrm{B}, \mathrm{P}, \mathrm{V}, \mathrm{Ni}, Z \geq 31$ & $\mathrm{Be}, \mathrm{B}, \mathrm{P}, \mathrm{V}, \mathrm{Ni}, Z \geq 31$ \\
\hline 1D LTE (literature) & remaining elements & $\mathrm{F}, \mathrm{Cl}, \mathrm{In}, \mathrm{Th}$ & $\mathrm{F}, \mathrm{Cl}, \mathrm{In}, \mathrm{Th}$ \\
\hline
\end{tabular}

non-LTE line formation of C, N, and O in the Sun (Amarsi et al. 2018a, 2019, 2020). This is an updated version of the Stagger solar model used in Asplund et al. (2009), Scott et al. (2015a,b), and Grevesse et al. (2015), but the differences in photospheric structure are minor. Detailed 3D radiative transfer with lineblanketing through the opacity binning technique (Nordlund 1982; Collet et al. 2018) is included under the assumption of LTE to compute the necessary radiative heating and cooling rates in the photosphere. The resulting temperature stratification is highly realistic, as is evident from comparisons with the observed continuum centre-to-limb variation (Amarsi et al. 2018a); this was also the case with the 3D model used in Asplund et al. (2009). The predictions of our 3D solar models are in excellent agreement with all key observational diagnostics (e.g., Pereira et al. 2013). The resulting effective temperature of the $24 \mathrm{~h}$ simulation sequence is $5773 \pm 16 \mathrm{~K}$ (standard deviation), indistinguishable from the solar value of $5772 \mathrm{~K}$ (Prša et al. 2016). The 3D Stagger models employ an updated version of the so-called MHD (named after the authors) equation of state (Mihalas et al. 1988; Trampedach et al. 2013), continuous and line opacities from the MARCS package (Gustafsson et al. 2008; Hayek et al. 2010), and the solar chemical composition of Asplund et al. (2009). No magnetic fields were included. From the $240^{3}$ simulation grid covering $6 \mathrm{Mm}$ horizontally and $3 \mathrm{Mm}$ vertically, a resampled $3 \mathrm{D}$ grid covering the photosphere $(1 \mathrm{Mm}$ vertically) was interpolated before the spectral line formation calculations for the abundance determinations to improve the numerical accuracy and to save computational time without sacrificing accuracy; the grid resolutions of both the original and interpolated solar models are sufficient to obtain the correct spectral line broadening from convective motions and oscillations (Asplund et al. 2000a,b; Pereira et al. 2013).

To quantify various systematic uncertainties, we also made use of several 1D model atmospheres: the temporally and spatially averaged 3D model (referred to here as $\langle 3 \mathrm{D}\rangle$ ); a 1D hydrostatic ATMO model with mixing length theory convection and identical equation of state and opacities to the 3D model (Magic et al. 2013, Appendix A); a 1D hydrostatic model computed with the widely used MARCS code (Gustafsson et al. 2008); and the 1D semi-empirical Holweger \& Müller (1974) model, which has traditionally been the model of choice for solar abundance analyses for the past half-century.

Spectral line formation. For key atoms and ions, the results are based on 3D non-LTE calculations, using the MPIparallelised Balder code (Amarsi et al. 2018b), our extensively modified version of Multi3D (Leenaarts \& Carlsson 2009). For Li I, C I, N I, Al I, and Si I/Si II, these results were adopted from recently published studies (Wang et al. 2021; Amarsi et al. 2019, 2020; Nordlander \& Lind 2017; Amarsi \& Asplund 2017), while for O I, Na I, Mg I/Mg II, K I, Ca I/Ca II, and Fe I/Fe II, they are based on new calculations. We also adopted 3D non-LTE literature results for Ba II, which are based on Multi3D calculations (Gallagher et al. 2020).

Balder iteratively solves the 3D (or 1D) radiative transfer equation coupled with the equations for statistical equilibrium (i.e. the atomic level populations fulfil $\partial n_{\mathrm{i}} / \partial t=0$ ) under the assumption that the departures from LTE for an element do not feed back onto the atmospheric structure, nor do they impact the line formation of other elements. Balder employs a comprehensive equation of state, background continuum, and line opacities calculated with the Blue code as described in Amarsi et al. (2016b), with the present work adopting the occupation probability formalism to better model line opacities near the series limits (Hubeny et al. 1994). The 3D non-LTE radiative transfer is solved iteratively for typically 5-10 snapshots of the 3D solar model in 26 directions using short characteristics (Ibgui et al. 2013). The number of frequencies for which the radiative transfer equation needs to be computed to adequately resolve the bound-free and bound-bound transitions varies among elements, but for our most complex model atoms $(\mathrm{Ca}$ and $\mathrm{Fe})$ it amounts to 20000 and 45000 , respectively. After convergence, a final 
Table 2. Present-day solar photospheric abundances together with those in CI chondrites (Palme et al. 2014), renormalised to the solar Si abundance (Sect. 3).

\begin{tabular}{|c|c|c|c|c|c|c|c|c|c|}
\hline $\mathrm{Z}$ & Element & Photosphere & CI chondrites & Comments & $\mathrm{Z}$ & Element & Photosphere & CI chondrites & Comments \\
\hline 1 & $\mathrm{H}$ & $12.00 \pm 0.00$ & $8.22 \pm 0.04$ & definition & 44 & $\mathrm{Ru}$ & $1.75 \pm 0.08$ & $1.77 \pm 0.02$ & \\
\hline 2 & $\mathrm{He}$ & $10.914 \pm 0.013$ & $1.29 \pm 0.18$ & helioseismology & 45 & $\mathrm{Rh}$ & $0.78 \pm 0.11$ & $1.04 \pm 0.02$ & \\
\hline 3 & $\mathrm{Li}$ & $0.96 \pm 0.06$ & $3.25 \pm 0.04$ & meteorites & 46 & $\mathrm{Pd}$ & $1.57 \pm 0.10$ & $1.65 \pm 0.02$ & \\
\hline 4 & $\mathrm{Be}$ & $1.38 \pm 0.09$ & $1.32 \pm 0.03$ & & 47 & $\mathrm{Ag}$ & $0.96 \pm 0.10$ & $1.20 \pm 0.04$ & \\
\hline 5 & B & $2.70 \pm 0.20$ & $2.79 \pm 0.04$ & & 48 & $\mathrm{Cd}$ & & $1.71 \pm 0.03$ & meteorites \\
\hline 6 & $\mathrm{C}$ & $8.46 \pm 0.04$ & $7.39 \pm 0.04$ & & 49 & In & $0.80 \pm 0.20$ & $0.76 \pm 0.02$ & \\
\hline 7 & $\mathrm{~N}$ & $7.83 \pm 0.07$ & $6.26 \pm 0.06$ & & 50 & $\mathrm{Sn}$ & $2.02 \pm 0.10$ & $2.07 \pm 0.06$ & \\
\hline 8 & $\mathrm{O}$ & $8.69 \pm 0.04$ & $8.39 \pm 0.04$ & & 51 & $\mathrm{Sb}$ & & $1.01 \pm 0.06$ & meteorites \\
\hline 9 & $\mathrm{~F}$ & $4.40 \pm 0.25$ & $4.42 \pm 0.06$ & & 52 & $\mathrm{Te}$ & & $2.18 \pm 0.03$ & meteorites \\
\hline 10 & $\mathrm{Ne}$ & $8.06 \pm 0.05$ & $-1.12 \pm 0.18$ & solar wind & 53 & I & & $1.55 \pm 0.08$ & meteorites \\
\hline 11 & $\mathrm{Na}$ & $6.22 \pm 0.03$ & $6.27 \pm 0.04$ & & 54 & $\mathrm{Xe}$ & $2.22 \pm 0.05$ & $-1.95 \pm 0.18$ & nuclear physics \\
\hline 12 & $\mathrm{Mg}$ & $7.55 \pm 0.03$ & $7.53 \pm 0.02$ & & 55 & $\mathrm{Cs}$ & & $1.08 \pm 0.03$ & meteorites \\
\hline 13 & $\mathrm{Al}$ & $6.43 \pm 0.03$ & $6.43 \pm 0.03$ & & 56 & $\mathrm{Ba}$ & $2.27 \pm 0.05$ & $2.18 \pm 0.02$ & \\
\hline 14 & $\mathrm{Si}$ & $7.51 \pm 0.03$ & $7.51 \pm 0.01$ & & 57 & $\mathrm{La}$ & $1.11 \pm 0.04$ & $1.17 \pm 0.01$ & \\
\hline 15 & $\mathrm{P}$ & $5.41 \pm 0.03$ & $5.43 \pm 0.03$ & & 58 & $\mathrm{Ce}$ & $1.58 \pm 0.04$ & $1.58 \pm 0.01$ & \\
\hline 16 & $\mathrm{~S}$ & $7.12 \pm 0.03$ & $7.15 \pm 0.02$ & & 59 & $\operatorname{Pr}$ & $0.75 \pm 0.05$ & $0.76 \pm 0.01$ & \\
\hline 17 & $\mathrm{Cl}$ & $5.31 \pm 0.20$ & $5.23 \pm 0.06$ & & 60 & $\mathrm{Nd}$ & $1.42 \pm 0.04$ & $1.45 \pm 0.01$ & \\
\hline 18 & $\mathrm{Ar}$ & $6.38 \pm 0.10$ & $-0.50 \pm 0.18$ & solar wind & 62 & $\mathrm{Sm}$ & $0.95 \pm 0.04$ & $0.94 \pm 0.01$ & \\
\hline 19 & $\mathrm{~K}$ & $5.07 \pm 0.03$ & $5.08 \pm 0.04$ & & 63 & $\mathrm{Eu}$ & $0.52 \pm 0.04$ & $0.52 \pm 0.01$ & \\
\hline 20 & $\mathrm{Ca}$ & $6.30 \pm 0.03$ & $6.29 \pm 0.03$ & & 64 & $\mathrm{Gd}$ & $1.08 \pm 0.04$ & $1.05 \pm 0.01$ & \\
\hline 21 & $\mathrm{Sc}$ & $3.14 \pm 0.04$ & $3.04 \pm 0.03$ & & 65 & $\mathrm{~Tb}$ & $0.31 \pm 0.10$ & $0.31 \pm 0.01$ & \\
\hline 22 & $\mathrm{Ti}$ & $4.97 \pm 0.05$ & $4.90 \pm 0.03$ & & 66 & Dy & $1.10 \pm 0.04$ & $1.13 \pm 0.01$ & \\
\hline 23 & $\mathrm{~V}$ & $3.90 \pm 0.08$ & $3.96 \pm 0.03$ & & 67 & Ho & $0.48 \pm 0.11$ & $0.47 \pm 0.01$ & \\
\hline 24 & $\mathrm{Cr}$ & $5.62 \pm 0.04$ & $5.63 \pm 0.02$ & & 68 & $\mathrm{Er}$ & $0.93 \pm 0.05$ & $0.93 \pm 0.01$ & \\
\hline 25 & $\mathrm{Mn}$ & $5.42 \pm 0.06$ & $5.47 \pm 0.03$ & & 69 & $\mathrm{Tm}$ & $0.11 \pm 0.04$ & $0.12 \pm 0.01$ & \\
\hline 26 & $\mathrm{Fe}$ & $7.46 \pm 0.04$ & $7.46 \pm 0.02$ & & 70 & $\mathrm{Yb}$ & $0.85 \pm 0.11$ & $0.92 \pm 0.01$ & \\
\hline 27 & Co & $4.94 \pm 0.05$ & $4.87 \pm 0.02$ & & 71 & $\mathrm{Lu}$ & $0.10 \pm 0.09$ & $0.09 \pm 0.01$ & \\
\hline 28 & $\mathrm{Ni}$ & $6.20 \pm 0.04$ & $6.20 \pm 0.03$ & & 72 & $\mathrm{Hf}$ & $0.85 \pm 0.05$ & $0.71 \pm 0.01$ & \\
\hline 29 & $\mathrm{Cu}$ & $4.18 \pm 0.05$ & $4.25 \pm 0.06$ & & 73 & $\mathrm{Ta}$ & & $-0.15 \pm 0.04$ & meteorites \\
\hline 30 & $\mathrm{Zn}$ & $4.56 \pm 0.05$ & $4.61 \pm 0.02$ & & 74 & W & $0.79 \pm 0.11$ & $0.65 \pm 0.04$ & \\
\hline 31 & $\mathrm{Ga}$ & $3.02 \pm 0.05$ & $3.07 \pm 0.03$ & & 75 & $\mathrm{Re}$ & & $0.26 \pm 0.02$ & meteorites \\
\hline 32 & $\mathrm{Ge}$ & $3.62 \pm 0.10$ & $3.58 \pm 0.04$ & & 76 & Os & $1.35 \pm 0.12$ & $1.35 \pm 0.02$ & \\
\hline 33 & As & & $2.30 \pm 0.04$ & meteorites & 77 & $\mathrm{Ir}$ & & $1.32 \pm 0.02$ & meteorites \\
\hline 34 & $\mathrm{Se}$ & & $3.34 \pm 0.03$ & meteorites & 78 & $\mathrm{Pt}$ & & $1.61 \pm 0.02$ & meteorites \\
\hline 35 & $\mathrm{Br}$ & & $2.54 \pm 0.06$ & meteorites & 79 & $\mathrm{Au}$ & $0.91 \pm 0.12$ & $0.81 \pm 0.05$ & \\
\hline 36 & $\mathrm{Kr}$ & $3.12 \pm 0.10$ & $-2.27 \pm 0.18$ & solar wind & 80 & $\mathrm{Hg}$ & & $1.17 \pm 0.18$ & meteorites \\
\hline 37 & $\mathrm{Rb}$ & $2.32 \pm 0.08$ & $2.37 \pm 0.03$ & & 81 & $\mathrm{Tl}$ & $0.92 \pm 0.17$ & $0.77 \pm 0.05$ & \\
\hline 38 & $\mathrm{Sr}$ & $2.83 \pm 0.06$ & $2.88 \pm 0.03$ & & 82 & $\mathrm{~Pb}$ & $1.95 \pm 0.08$ & $2.03 \pm 0.03$ & \\
\hline 39 & $\mathrm{Y}$ & $2.21 \pm 0.05$ & $2.15 \pm 0.02$ & & 83 & $\mathrm{Bi}$ & & $0.65 \pm 0.04$ & meteorites \\
\hline 40 & $\mathrm{Zr}$ & $2.59 \pm 0.04$ & $2.53 \pm 0.02$ & & 90 & $\mathrm{Th}$ & $0.03 \pm 0.10$ & $0.04 \pm 0.03$ & \\
\hline 41 & $\mathrm{Nb}$ & $1.47 \pm 0.06$ & $1.42 \pm 0.04$ & & 92 & $\mathrm{U}$ & & $-0.54 \pm 0.03$ & meteorites \\
\hline 42 & Mo & $1.88 \pm 0.09$ & $1.93 \pm 0.04$ & & & & & & \\
\hline
\end{tabular}

Notes. Elements that are commented on are those for which the adopted proto-solar abundances (Sect. 5) are not based on spectroscopy of the solar photosphere; we caution that the differences between solar and CI abundances show a trend with condensation temperature (Sect. 3.3), which should be taken into account when estimating the Solar System composition.

formal solution is carried out for the emergent vertical intensity and for the spectral lines of interest, using a long characteristics solver and higher frequency resolution for accuracy.

Other atomic and ionic lines, as well as all molecular lines, are based on 3D LTE spectrum synthesis using the SCATE code (Hayek et al. 2010), for the most part as presented in our earlier papers (Scott et al. 2015a,b; Grevesse et al. 2015). New 3D LTE calculations were adopted for molecular species of $\mathrm{C}, \mathrm{N}$, and $\mathrm{O}$. When no 3D non-LTE calculations had been performed, we relied on our own or published 1D non-LTE abundance corrections, if possible; this procedure is not fully consistent but is expected to be more realistic than the LTE results. Many elements, in particular those beyond the Fe peak, unfortunately still lack non-LTE studies, requiring us to adopt the 3D LTE results. It is emphasised that no micro- and macro-turbulence enter the 3D calculations (Asplund et al. 2000b); for the comparison 1D spectrum syntheses, we assumed a microturbulence of $1 \mathrm{~km} \mathrm{~s}^{-1}$ (e.g., Holweger et al. 1978; Steffen et al. 2013).

Atomic data. The computation of the theoretical solar spectrum hinges critically on the availability of accurate atomic data. We have scoured and carefully assessed the literature and databases for the most up-to-date and most reliable input data required for our solar modelling: transition probabilities, hyperfine splitting, spectral line broadening, partition functions, excitation, ionisation, and dissociation energies, etc (e.g., 
Piskunov et al. 1995; Barklem \& Collet 2016; Heiter et al. 2021). In most cases, experimental data have been selected over theoretical data, with the NIST Atomic Spectra Database ${ }^{4}$ (Kramida 2019) providing much of the quantitative assessment of the uncertainties in $g f$-values. For the solar photosphere, the continuous opacities are well determined, except possibly in the UV, and hence they are not a major concern. For the non-LTE calculations, we also made extensive use of the bound-bound and boundfree radiative data from the Opacity Project, the Iron Project, and other dedicated atomic calculations (e.g., Seaton 1987; Hummer et al. 1993; Badnell et al. 2005), as well as theoretical cross-sections for collisions with electrons and with neutral hydrogen (e.g., Bray \& Stelbovics 1992, 1995; Zatsarinny 2006; Zatsarinny \& Bartschat 2013; Barklem 2016b; Barklem et al. 2017; Belyaev \& Yakovleva 2017). Fortunately the situation today in terms of the necessary atomic and molecular data for solar/stellar spectroscopy is significantly improved compared to just a decade ago thanks to the tireless contributions from a relatively small group of atomic physicists to whom we are greatly indebted.

Observations. To derive the solar elemental abundances, the predicted solar spectrum was compared to the observed highresolution disc-centre intensity spectrum of the quiet Sun with a preference for the Liège (named after its location of production, not observations which were done at Jungfraujoch) visual solar atlas (Delbouille et al. 1973) due to its low degree of contamination of telluric absorption lines. We also made use of other solar atlases for the disc-centre intensity (Neckel 1999; Kurucz 2005), the disc-integrated flux (Kurucz et al. 1984; Kurucz 2006; Reiners et al. 2016), as well as observations in the infrared (Delbouille et al. 1981; Farmer \& Norton 1989; Abrams et al. 1996; Hase et al. 2010) as needed and for evaluation purposes; disc-centre intensity spectra are in general preferable to flux to disentangle the contribution of blends. In some cases, we also employ the high-resolution optical centre-to-limb observations of Pereira et al. (2009) and Stenflo (2015) to verify the correctness of our line formation calculations. All of these observed solar spectra have extremely high resolving power (typically equivalent of $R \equiv \lambda / \Delta \lambda>200,000)$ and signal-to-noise (mostly $S / N>1000)$. We either perform a line profile fitting using standard $\chi^{2}$-techniques or match the observed and computed equivalent widths, depending on the line and possible presence of blends. We allowed our own local continuum placement around the spectral lines in question, as appropriate. Doerr et al. (2016) and Reiners et al. (2016) examined the differences between some of these atlases and overall find excellent agreement; our results are not significantly affected by the particular choice of observations except for a few individual lines.

Uncertainties. Homogenously quantifying the uncertainties of elemental abundances, including systematic errors is almost as important as having accurate determinations of them. Concerning elements for which multiple spectral lines are available for the abundance analysis, we used the weighted standard error of the mean as the statistical uncertainty, the weights being our assessment of the quality of the lines. In cases when only one or two lines can be used, we estimated the statistical error from the goodness of the profile fitting or equivalent width measurement uncertainty.

Following Asplund et al. (2009), we devoted much effort to carefully investigating and evaluating possible systematic

\footnotetext{
4 https://physics.nist.gov/PhysRefData/ASD/lines_form. html
}

errors in the analysis, in particular related to the 3D model atmosphere and 3D non-LTE line formation. To quantify the errors from shortcomings in the photosphere temperature structure we take half the differences between the derived abundances from the $\langle 3 \mathrm{D}\rangle$ model and the Holweger \& Müller (1974) model; as noted above, we know from the centre-to-limb variation that the $\langle 3 \mathrm{D}\rangle$ model is a much closer match to the real Sun (Asplund et al. 2009; Pereira et al. 2013) with newer semiempirical solar model atmospheres (Grevesse \& Sauval 1998; Allende Prieto et al. 2001a) having 50-100 K lower temperatures than the Holweger \& Müller (1974) model in the lineforming region, which is very similar to our $3 \mathrm{D}$ model. The impact of atmospheric inhomogeneities is evaluated from taking half the difference between the $3 \mathrm{D}$ and $\langle 3 \mathrm{D}\rangle$ results, which is justified by the close resemblance between the predicted and observed solar granulation patterns. In general, the systematic uncertainty due to non-LTE line formation is estimated as half of the predicted non-LTE abundance correction (3D non-LTE 3D LTE, or 1D non-LTE - 1D LTE) with a minimum error of 0.03 dex when no non-LTE calculations are available; an exception is made for $\mathrm{O}$ for which the non-LTE effects can be accurately quantified using, for example, the centre-to-limb variation of the OI $777 \mathrm{~nm}$ triplet and therefore the uncertainties would otherwise be significantly overestimated. These three systematic errors are combined in quadrature with the statistical error to compute the total abundance error.

For some elements, we tried to factor in uncertainties in the input atomic data but in general this has not been attempted due to those often being poorly known and the huge amount of atomic data required for non-LTE modelling. While impossible to know without even more sophisticated solar modelling, we expect our estimated uncertainties to be realistic and possibly conservative overall, although we caution that in a few cases, especially in the absence of any non-LTE calculations, the errors are likely underestimated; in cases where they were particularly striking, we artificially inflated the computed errors somewhat, as noted.

\subsection{Light elements: Lithium to beryllium}

$\mathrm{Li}, \mathrm{Be}$, and $\mathrm{B}$ were not analysed in our previous series of papers devoted to the solar chemical composition (Scott et al. 2015a,b; Grevesse et al. 2015) and are therefore discussed explicitly here.

Lithium $(Z=3)$. In practise, the solar Li abundance can only be determined from the very weak Li I $670.8 \mathrm{~nm}$ resonance line, an undertaking that is further complicated by the presence of several blending lines of $\mathrm{CN}, \mathrm{Fe}$, and other species. We adopted the recently revised solar Li abundance from Wang et al. (2021), who presented new 3D non-LTE spectral line formation calculations for a wide range of stellar parameters, including for the Sun. Importantly, their more complete treatment of background line opacities in the UV compared with previous studies (e.g., Carlsson et al. 1994; Asplund et al. 2003; Lind et al. 2009, 2013; Harutyunyan et al. 2018; Mott et al. 2020) significantly changes the statistical equilibrium balance of Li I, leading in general to a strengthening of the $670.8 \mathrm{~nm}$ line. Using their new 3D non-LTE calculations and allowing for blends, Wang et al. (2021) derived a solar $\mathrm{Li}$ abundance of $\log \epsilon_{\mathrm{Li}}=0.96 \pm 0.03$ (statistical) \pm 0.05 (systematic) and a $0.09 \mathrm{dex}(23 \%)$ reduction compared to the advocated value in Asplund et al. (2009).

Beryllium $(Z=4)$. Following Chmielewski et al. (1975), it was widely believed that Be was depleted by a factor of two in the solar photosphere compared with CI chondrites. This 
conclusion was first challenged by Balachandran \& Bell (1998) who demonstrated that missing UV opacity (e.g., Bell et al. 2001; Short \& Hauschildt 2009) in the stellar models leads to an underestimated solar Be abundance when relying on the Be II $313 \mathrm{~nm}$ resonance lines. By calibrating the missing opacity in this wavelength region by enforcing the same $\mathrm{O}$ abundance to be estimated from nearby UV OH lines with $\mathrm{OH}$ lines in the IR, they found a solar photospheric Be abundance consistent with the meteoritic value. Their conclusion was corroborated in a $3 \mathrm{D}$ LTE analysis by Asplund (2004), which we adopted ( $\log \epsilon_{\mathrm{Be}}=$ $1.38 \pm 0.09$; statistical + systematic), suggesting no solar Be depletion. We caution, however, that unaccounted-for blends in this crowded UV region may have biased the inferred Be abundance upwards. Revisiting the topic with 3D non-LTE calculations and a careful assessment of blending lines and continuum placement together with a detailed evaluation of any missing UV opacity would be worthwhile to finally settle the issue, especially given the importance of accurately constraining the amount of mixing below the solar convection zone (Sect. 3.2).

Boron $(Z=5)$. The solar B abundance can in practice only be inferred from the B I $249.7 \mathrm{~nm}$ resonance line, which makes the result quite uncertain due to the heavy crowding and possible missing opacity in this wavelength region. The most recent spectroscopic analysis is that of Cunha \& Smith (1999), who inferred $\log \epsilon_{\mathrm{B}}=2.70 \pm 0.20$ from a 1D LTE spectrum synthesis, which we have consequently adopted here as the present-day photospheric abundance. While the minority ionisation stage and low excitation potential may suggest that the $249.7 \mathrm{~nm}$ line should be susceptible to severe departures from LTE, this does not seem to be the case for the Sun due to a somewhat fortuitous cancellation of non-LTE effects in 1D (Kiselman 1994; Tan et al. 2010). No 3D non-LTE study for B exists to our knowledge.

\subsection{Carbon, nitrogen, and oxygen}

The fourth, sixth, and third most abundant elements in the Sun by number, $\mathrm{C}, \mathrm{N}$, and $\mathrm{O}$ are incredibly important to modern astronomy. They also play a central role in the outstanding solar modelling problem (e.g., Basu \& Antia 2008; Zhang et al. 2019), wherein a highly significant discrepancy exists pertaining to the interior structure of the Sun as inferred precisely via helioseismic inversions and standard solar models (see Sect. 6). O is the dominant source of opacity at the most problematic region, namely the base of the convection zone, with $\mathrm{C}$ and $\mathrm{N}$ also providing a non-negligible contribution there. It has been noted that higher $\mathrm{C}, \mathrm{N}$, and (especially) $\mathrm{O}$ abundances by $\approx 0.15 \mathrm{dex}$ would alleviate the discrepancy with the sound speed (e.g., Pinsonneault \& Delahaye 2009), but this would not address all observational constraints (Buldgen et al. 2019b). Adding to the controversy, all three elements form volatile compounds and are thus depleted in meteorites; their abundances must be inferred through spectroscopy of the solar photosphere.

Parallel to our work, the CO ${ }^{5}$ BOLD group have led a commendable effort to measure the solar $\mathrm{C}, \mathrm{N}$, and $\mathrm{O}$ abundances from atomic lines, similarly employing $3 \mathrm{D}$ hydrodynamic model atmospheres (Caffau et al. 2008, 2009, 2010, 2013, 2015; Steffen et al. 2015); they have not yet explored the molecular lines. These studies tend to find slightly higher $\mathrm{C}, \mathrm{N}$, and $\mathrm{O}$ abundances by 0.03 0.07 dex that usually agree within the uncertainties (Caffau et al. 2011). These differences most likely do not originate in the $3 \mathrm{D}$ models or radiative transfer codes themselves, but rather can be traced to our more conservative choice of unblended lines, the adopted oscillator strengths, and the measured equivalent widths, as has been demonstrated for C (Amarsi et al. 2019) and $\mathrm{N}$ (Amarsi et al. 2020); moreover, unpublished calculations have similarly indicated good agreement for the O I $777 \mathrm{~nm}$ triplet when adopting exactly the same input data.

After submission of our article, we became aware of new 3D non-LTE line formation calculations of the O I $777 \mathrm{~nm}$ triplet and [O I] $630 \mathrm{~nm}$ forbidden line by Bergemann et al. (2021) using 3D solar models computed with the Stagger and Bifrost codes, the latter with and without a chromosphere. Their results agree perfectly with those presented here and in Asplund et al. (2009): $\log \epsilon_{\mathrm{OI} 777}=8.70 \pm 0.05$ and $\log \epsilon_{[\mathrm{OI}] 630}=8.71 \pm 0.03$, further supporting a low solar $\mathrm{O}$ abundance. Reassuringly, they find only a very minor effect $(\approx 0.01 \mathrm{dex})$ of a chromospheric temperature rise on these lines.

An extension to using a 1D semi-empirical model atmosphere has recently been presented by Cubas Armas et al. (2017, 2020), who derived a 3D solar model from the spectral inversion of three spatially resolved Fe I lines. They applied it to the [O I] $630.0 \mathrm{~nm}$ line in the Sun to infer $\log \epsilon_{\mathrm{O}}=8.80 \pm 0.03$. While this is an interesting approach well worth pursuing further, we consider it premature to adopt their derived abundance until this empirical 3D solar model has been exposed to a variety of observational tests, which our theoretical 3D hydrodynamical models have successfully passed (Nordlund et al. 2009; Pereira et al. 2013). In particular, it is not clear whether their model has the appropriate $T_{\text {eff }}$ since it is neither an imposed constraint of the spectral inversion of the normalised line profiles, nor is it tested for afterwards. It remains to be seen whether consistent $\mathrm{O}$ abundances would be obtained from the [O I], O I, and $\mathrm{OH}$ lines.

Table 3 shows the results of our reanalysis of all the available indicators of $\mathrm{C}, \mathrm{N}$, and $\mathrm{O}$ using our most recent $3 \mathrm{D}$ model atmosphere and the most up-to-date atomic and molecular data. The atomic results are based on recent 3D nonLTE studies for C (Amarsi et al. 2019), N (Amarsi et al. 2020), and $\mathrm{O}$ (Amarsi et al. 2018a); in the latter case, the calculations were extended to include the other available atomic indicators (see also Amarsi 2016). The molecular results come from a new homogeneous analysis of 879 different lines, with the background $\mathrm{C}, \mathrm{N}$, and $\mathrm{O}$ abundances iterated to consistency (Amarsi et al. 2021). Compared to Asplund et al. (2009), the new recommended abundances are identical for $\mathrm{N}$ and $\mathrm{O}$ and only 0.03 dex higher for $\mathrm{C}$.

Carbon $(Z=6)$. Of the three elements considered in this section, $C$ has the largest number of available indicators (Table 3 ) and is arguably the best constrained. The [C I ] result is drawn from the 3D non-LTE analysis of the $872.7 \mathrm{~nm}$ line from Amarsi et al. (2019), although we note that this low-excitation line is wellreproduced in 3D LTE. The $\mathrm{C}$ I result is based on the 14 lines also analysed in this 3D non-LTE study; here, the result has increased by $0.03 \mathrm{dex}$ in light of new $g f$-values from large-scale atomic structure calculations by Li et al. (2021). The molecular results are based on new 3D LTE calculations of 39 lines in the $\mathrm{C}_{2}$ Swan system, and $\mathrm{CH}$ lines which were divided into 51 fundamental rovibrational $(\mathrm{d} v=1)$ lines and seven electronic lines in the $\mathrm{CH}$ A-X system. $\mathrm{CO}$ lines were also considered by adopting the $\mathrm{O}$ abundance inferred from $\mathrm{OH}$ lines for a particular model atmosphere and including the error in this $\mathrm{O}$ abundance in the total error budget. The $\mathrm{CO}$ lines were divided into two groups of rovibrational transitions, including 28 belonging to fundamental $(\mathrm{d} v=$ $1)$ bands and 52 to first overtone $(\mathrm{d} v=2)$ bands.

The advocated solar $\mathrm{C}$ abundance is $8.46 \pm 0.04$. This was evaluated as the weighted mean and error of the $[\mathrm{CI}], \mathrm{CI}$, $\mathrm{C}_{2}, \mathrm{CH}$, and $\mathrm{CO}$ results given in Table 3; to avoid giving 
Table 3. C, N, and $\mathrm{O}$ abundances in the solar photosphere as inferred from the available indicators and from different model atmospheres, with the combined statistical and systematic uncertainties in the final column.

\begin{tabular}{|c|c|c|c|c|c|c|c|c|c|}
\hline \multirow[t]{2}{*}{ Indicator } & \multicolumn{4}{|c|}{ Non-LTE } & \multicolumn{4}{|c|}{ LTE } & \multirow[t]{2}{*}{ Error } \\
\hline & $3 \mathrm{D}$ & $\langle 3 \mathrm{D}\rangle$ & MARCS & $\mathrm{HM}$ & $3 \mathrm{D}$ & $\langle 3 \mathrm{D}\rangle$ & MARCS & $\mathrm{HM}$ & \\
\hline$[\mathrm{CI}]$ & 8.453 & 8.424 & 8.421 & 8.432 & 8.453 & 8.424 & 8.421 & 8.432 & 0.034 \\
\hline $\mathrm{CI}$ & 8.470 & 8.459 & 8.456 & 8.488 & 8.480 & 8.467 & 8.462 & 8.497 & 0.038 \\
\hline $\mathrm{C}_{2}$ Swan & - & - & - & - & 8.455 & 8.473 & 8.433 & 8.520 & 0.029 \\
\hline $\mathrm{CH}(\mathrm{A}-\mathrm{X})$ & - & - & - & - & 8.459 & 8.471 & 8.393 & 8.555 & 0.048 \\
\hline $\mathrm{CH}(\mathrm{d} v=1)$ & - & - & - & - & 8.470 & 8.503 & 8.438 & 8.573 & 0.043 \\
\hline $\mathrm{CO}(\mathrm{d} v=1)$ & - & - & - & - & 8.487 & 8.653 & 8.606 & 8.683 & 0.101 \\
\hline $\mathrm{CO}(\mathrm{d} v=2)$ & - & - & - & - & 8.467 & 8.681 & 8.609 & 8.743 & 0.122 \\
\hline$\overline{\mathrm{N}} \mathrm{I}$ & 7.767 & 7.816 & 7.804 & 7.864 & 7.780 & 7.828 & 7.813 & 7.879 & 0.035 \\
\hline $\mathrm{NH}(\mathrm{d} v=0)$ & - & - & - & - & 7.881 & 8.032 & 8.021 & 8.079 & 0.087 \\
\hline $\mathrm{NH}(\mathrm{d} v=1)$ & - & - & - & - & 7.921 & 7.988 & 7.914 & 8.056 & 0.050 \\
\hline $\mathrm{CN}(0-0)$ & - & - & - & - & 7.867 & 7.964 & 7.927 & 8.016 & 0.072 \\
\hline $\mathrm{CN}(\mathrm{d} v \geq 1)$ & - & - & - & - & 7.899 & 7.994 & 7.959 & 8.046 & 0.078 \\
\hline$[\mathrm{O} \mathrm{I}]$ & 8.703 & 8.688 & 8.664 & 8.705 & 8.703 & 8.688 & 8.664 & 8.705 & 0.051 \\
\hline O I & 8.686 & 8.682 & 8.657 & 8.678 & 8.805 & 8.779 & 8.732 & 8.789 & 0.030 \\
\hline $\mathrm{OH}(\mathrm{d} v=0)$ & - & - & - & - & 8.690 & 8.792 & 8.782 & 8.837 & 0.070 \\
\hline $\mathrm{OH}(\mathrm{d} v=1)$ & - & - & - & - & 8.707 & 8.800 & 8.728 & 8.884 & 0.063 \\
\hline $\mathrm{OH}(\mathrm{d} v=2)$ & - & - & - & - & 8.690 & 8.756 & 8.673 & 8.848 & 0.087 \\
\hline
\end{tabular}

these species too much weight, the rovibrational and electronic results for $\mathrm{CH}$ were first combined into a weighted mean (8.46), as were the fundamental and first overtone results for $\mathrm{CO}$ (8.48). The final result is a 0.03 dex increase over those stated in Asplund et al. (2009). Nevertheless, the agreement between the different indicators found here is excellent, and noticeably improved over that earlier study. The reason for the new, slightly higher value can primarily be attributed to improvements to the atomic and molecular input data ([C I] Froese Fischer 2006; C I Li et al. 2021; $\mathrm{C}_{2}$ Brooke et al. 2013; CH Masseron et al. 2014; $\mathrm{CO} \mathrm{Li}$ et al. 2015), as well as to the employed molecular dissociation constants (Barklem \& Collet 2016).

Nitrogen $(Z=7)$. Compared to $\mathrm{C}$, there are relatively few good indicators of the solar $\mathrm{N}$ abundance. There are no [N I] lines visible in the solar spectrum, and only five N I lines were identified as being sufficiently strong and unblended to be measured with confidence (Amarsi et al. 2020). The 3D non-LTE atomic result is combined with new 3D LTE results for molecular lines (Table 3). This includes NH rovibrational lines that were divided into two groups consisting of 13 pure rotational $(\mathrm{d} v=0)$ and 15 fundamental $(\mathrm{d} v=1)$ lines. $\mathrm{CN}$ lines were also considered by adopting weighted mean carbon abundances as inferred from $\mathrm{C}_{2}, \mathrm{CH}$, and $\mathrm{CO}$ and for the different model atmospheres, and including the error in this $\mathrm{C}$ abundance in the total error budget. The $\mathrm{CN}$ lines were separated into two groups consisting of 59 electronic lines in the $0-0$ band, which typically has the best data, and 463 more lines with $\mathrm{d} v \geq 1$ in various bands.

Table 3 shows a discrepancy of 0.13 dex between the atomic $\mathrm{N}$ I result (7.77), and the weighted mean (7.90) of the mean $\mathrm{NH}$ (7.91) and $\mathrm{CN}$ (7.88) results. We can only speculate on where the inconsistency arises. The N I oscillator strengths were taken from Tachiev \& Froese Fischer (2002) and are expected to be reliable at the 0.03 dex level based on rankings from NIST (and verified with separate calculations as discussed in Sect. 2.1 of Amarsi et al. 2020). The oscillator strengths used here for $\mathrm{NH}$ and $\mathrm{CN}$ were taken from Brooke et al. (2014a,b, 2015), however it is difficult to imagine both sets of calculations having systematic offsets of 0.1 dex. Moreover, the dissociation energies of $\mathrm{CN}$ and NH are now well established (Barklem \& Collet 2016) and thus unlikely to be the reason behind the high molecular-based $\mathrm{N}$ abundance. We note that this discrepancy was already present in Asplund et al. (2009), albeit to a lesser degree, with the calculations there based on an unpublished set of older molecular data (A.J. Sauval, priv. comm.).

The advocated solar $\mathrm{N}$ abundance is $7.83 \pm 0.07$. Owing to the atomic versus molecular discrepancy, this was found by taking the unweighted mean of the atomic result together with the weighted mean of the molecular results. The uncertainty is given by the standard error in the mean (or equivalently, half the range between the two values). The value is unchanged from that of Asplund et al. (2009); however, the stipulated uncertainty is 0.02 dex larger.

Oxygen $(Z=8)$. The solar $\mathrm{O}$ abundance can be inferred from a number of different atomic and molecular indicators (Table 3 ). For the atomic indicators, we carried out a spectral line fitting analysis of the [OI] $557.7 \mathrm{~nm}, 630.0 \mathrm{~nm}$, and $636.4 \mathrm{~nm}$ lines, and of the OI $615.8 \mathrm{~nm}, 777 \mathrm{~nm}, 844.7 \mathrm{~nm}$, and $926.9 \mathrm{~nm}$ lines, using the 3D non-LTE model of Amarsi et al. (2018a). The fitting method follows that presented in Amarsi (2016, Chapter 4). Here, the strengths of the $\mathrm{CN}$ and $\mathrm{C}_{2}$ blending lines were fixed by adopting the line lists and abundances of the earlier $\mathrm{C}$ and $\mathrm{N}$ analyses and the strength of the blending $\mathrm{Ni}$ I $630 \mathrm{~nm}$ line calculated using the $g f$-value from Johansson et al. (2003) and the $\mathrm{Ni}$ abundance inferred here $\left(\log \epsilon_{\mathrm{Ni}}=6.20\right.$; Sect. 2.5). Ultimately, for [O I] nearly all weight was given to the $630.0 \mathrm{~nm}$ line after considering the relative difficulties in modelling the respective continua and blending contributions. Similarly for O I, most weight was given to the $777 \mathrm{~nm}$ triplet, because available centreto-limb observations (Pereira et al. 2009) allow us to correct for and constrain errors in the cross-sections for inelastic collisions with neutral hydrogen (following Amarsi et al. 2018a). The molecular results are based on new 3D LTE calculations of $\mathrm{OH}$ rovibrational lines, which were divided into three groups consisting of 84 pure rotational $(\mathrm{d} v=0), 50$ fundamental $(\mathrm{d} v=1)$, and 15 first overtone $(\mathrm{d} v=2)$ lines, all giving highly consistent results. 
The advocated solar $\mathrm{O}$ abundance is $8.69 \pm 0.04$. Our new value was determined from the weighted mean and error of the [O I], O I, and $\mathrm{OH}$ results given in Table 3, albeit first combining the three rovibrational $\mathrm{OH}$ results into a single weighted mean (8.70). An alternative value is obtained by taking the unweighted mean, either of these three species, or of all five indicators in Table 3. This would put less weight on the strong O I $777 \mathrm{~nm}$ triplet, but with both approaches the value is increased by less than $0.005 \mathrm{dex}$, since there is excellent agreement between the different indicators. Our recommended solar $\mathrm{O}$ abundance is unchanged from Asplund et al. (2009); however, the stipulated uncertainty is 0.01 dex smaller.

Combined with our revised $\mathrm{C}$ abundance, the solar $\mathrm{C} / \mathrm{O}$ ratio is $0.59 \pm 0.08$, a value which has steadily increased over the last few decades (e.g., 0.43: Anders \& Grevesse 1989; 0.48; Grevesse et al. 1991; 0.49: Grevesse \& Sauval 1998; 0.50: Allende Prieto et al. 2001b, 2002; 0.54: Asplund et al. 2004, 2005b; 0.55: Asplund et al. 2009; Caffau et al. 2011; 0.56: Amarsi et al. 2018a, 2019). The Sun has a higher C/O ratio than most solar twins regardless of age (Bedell et al. 2018), which could be a result of the Sun having migrated radially outwards by $2-3 \mathrm{kpc}$ in the Milky Way disc since birth (Nieva \& Przybilla 2012).

\subsection{Intermediate-mass elements: Sodium to calcium}

Sodium $(Z=11)$. The solar $\mathrm{Na}$ abundances presented in Asplund et al. (2009) and Scott et al. (2015b) were based on a 3D LTE analysis corrected for non-LTE effects using 1D model atmospheres (Lind et al. 2011). Here, we present the first full 3D non-LTE calculations for $\mathrm{Na}$ in the Sun, employing the same 23level $\mathrm{Na}$ model atom as in Lind et al. (2011), which importantly contains a full quantum mechanical description for inelastic $\mathrm{Na}$ I+H I collisions (Belyaev et al. 2010; Barklem et al. 2010). Experiments suggest these are sufficiently realistic for non-LTE modelling (Barklem et al. 2021).

A summary of the inferred abundances for the different lines using a variety of model atmospheres is provided in Table A.1. We employed the same five weak $\mathrm{Na}$ I lines as well as transition data as in Scott et al. (2015b); the equivalent widths were corrected for minor contributions from blending lines. The 3D non-LTE effects are minor $(\approx-0.03 \mathrm{dex})$ and very similar to those previously determined in $1 \mathrm{D}$. Our recommended 3D nonLTE $\mathrm{Na}$ abundance, based on the unweighted mean of the five $\mathrm{Na}$ I lines, becomes $\log \epsilon_{\mathrm{Na}}=6.22 \pm 0.01$ (statistical) \pm 0.03 (systematic), which is 0.01 dex larger than the value provided in Scott et al. (2015b).

Magnesium $(Z=12)$. Scott et al. (2015b) determined the solar photospheric $\mathrm{Mg}$ abundance based on 3D LTE line formation calculations coupled with non-LTE abundance corrections computed with different 1D model atmospheres. Their value, $\log \epsilon_{\mathrm{Mg}}=7.59 \pm 0.04$, was only 0.01 dex lower than the recommended abundance by Asplund et al. (2009). The Mg I and Mg II lines yielded fully consistent abundances in non-LTE, but they were significantly less so in LTE, highlighting the need for statistical equilibrium calculations for Mg II (non-LTE corrections are vanishingly small for $\mathrm{Mg}$ I for the Sun). Osorio et al. (2015) and Bergemann et al. (2017) carried out new 1D non-LTE calculations based on improved atomic data, but since their analyses were more focussed on other stars they have only very few lines in common with Scott et al. (2015b), most of their lines being much stronger in the Sun and less suitable for abundance purposes.
For the first time, we carried out full 3D non-LTE calculations of $\mathrm{Mg}$ in the solar photosphere. Our new atomic model consists of $48 \mathrm{Mg}$ I levels, $27 \mathrm{Mg}$ II levels, and the $\mathrm{Mg}$ III ground state; and 482 bound-bound and 71 bound-free radiative transitions, drawing on new experimental and theoretical transition probabilities for Mg I (Pehlivan Rhodin et al. 2017b). We made use of realistic cross-sections for inelastic collisions with electrons from Barklem et al. (2017) for Mg I and from Liang et al. (2009) for Mg II, and we used rate coefficients from Barklem et al. (2012) and Barklem (2016b) to describe inelastic collisions between $\mathrm{Mg}$ I and neutral hydrogen.

A summary of the inferred abundances for the different lines using a variety of model atmospheres is provided in Table A.1. The unweighted mean of eight $\mathrm{Mg}$ I lines gives $\log \epsilon_{\mathrm{Mg}}=7.56 \pm$ 0.01 (statistical) in 3D non-LTE. The systematic uncertainty as estimated from the 3D LTE, $\langle 3 \mathrm{D}\rangle$, and HM models is less than 0.01 dex as these all give highly consistent results for our $\mathrm{Mg}$ I lines. In 3D LTE, our Mg I result is 0.04 dex smaller than that given in Scott et al. (2015b) with the new $g f$-values only having a minor effect $(-0.01 \mathrm{dex})$. Instead, the difference stems mainly from how pressure broadening was treated; here, we used ABO theory (Anstee \& O'Mara 1995; Barklem \& O'Mara 1997; Barklem et al. 1998), adopting new and extended tables (P. S. Barklem, priv. comm. ${ }^{5}$ ). Accordingly, we increased the systematic uncertainty in the $\mathrm{Mg}$ I result to $0.02 \mathrm{dex}$.

The 3D non-LTE result from the unweighted mean of six lines of $\mathrm{Mg}$ II is significantly smaller: $\log \epsilon_{\mathrm{Mg}}=7.52 \pm 0.03$ (statistical). The Mg II results have a noticeably larger line-toline scatter, beyond the uncertainties in the adopted $g f$-values, which are all classified $\mathrm{A}$ or $\mathrm{A}+(\delta \log g f \leq \pm 0.01 \mathrm{dex})$ by NIST (Kramida 2019). The systematic uncertainty as estimated from the 3D LTE, $\langle 3 \mathrm{D}\rangle$, and HM models is 0.03 dex and is primarily driven by the large departures from LTE, in particular for three of the lines. Notably, we found that these lines are highly sensitive to the adopted recipe for inelastic collisions between $\mathrm{Mg}$ II and neutral hydrogen. Our results here are based on the Drawin (1968) recipe as formulated by Lambert (1993), without any adhoc scale factors applied, for lack of a better approach. Since the $3 \mathrm{D}$ LTE result is $\log \epsilon_{\mathrm{Mg}}=7.58$, more efficient collisions would help bring the $\mathrm{Mg}$ II result into better agreement with $\mathrm{Mg}$ I. Another uncertainty concerns the choice of $\mathrm{Mg}$ abundance for calculating the equation of state and hence electron pressures. Here, this abundance was varied consistently with the line formation calculations. An alternative approach would fix the $\mathrm{Mg}$ abundance to the value used to construct the model atmosphere $\left(\log \epsilon_{\mathrm{Mg}}=7.60\right)$ and vary the $g f$-values instead. Test calculations on the $\langle 3 \mathrm{D}\rangle$ model suggest that this alternative approach could increase the overall $\mathrm{Mg}$ II result by almost $0.02 \mathrm{dex}$; the electron pressure does not affect $\mathrm{Mg}$ I to first order since it is the minority ionisation stage in the solar photosphere.

Our recommended 3D non-LTE Mg abundance is the average of $\mathrm{Mg}$ I and $\mathrm{Mg}$ II, weighted by the total statistical and systematic errors: $\log \epsilon_{\mathrm{Mg}}=7.55 \pm 0.03$. A case could also be made for only adopting the $\mathrm{Mg}$ I results given the additional complications with $\mathrm{Mg}$ II, but the final abundance would only be 0.01 dex higher.

Aluminium $(Z=13)$. Nordlander \& Lind (2017) recently performed full 3D non-LTE spectral line formation calculations for Al I lines in the Sun, based on their new model atom that includes realistic cross-sections for inelastic collisions with neutral hydrogen (Belyaev 2013). They employed the same 3D hydrodynamical Stagger model solar atmosphere and 3D non-LTE radiative

\footnotetext{
https://github.com/barklem/abo-cross
} 
transfer code Balder as we employed in this study. Impressively, their solar modelling also agrees well with the observed IR emission lines of Al I. They adopted the same lines and line data as in Scott et al. (2015b), except that they excluded the $1089.1 \mathrm{~nm}$ line due to telluric contamination, finding a solar photospheric $\mathrm{Al}$ abundance of $\log \epsilon_{\mathrm{Al}}=6.43 \pm 0.03$ (statistical + systematic). Here, we adopted the Nordlander \& Lind (2017) solar abundance, which is identical to the 3D LTE results of Scott et al. (2015b); for the Sun, departures from LTE are in fact largely unimportant for these five Al I lines.

Silicon $(Z=14)$. Here, we adopted the solar Si abundance from our recent analysis (Amarsi \& Asplund 2017), the first full 3D non-LTE line formation calculations performed for this element. The same atomic data and line weighting as in Scott et al. (2015b) were used for the nine Si I and one Si II lines. The inferred photospheric Si abundance $\left(\log \epsilon_{\mathrm{Si}}=7.51 \pm 0.03\right)$ is identical to the values found by Asplund et al. (2009) and Scott et al. (2015b); this value is used to normalise the meteoritic abundance scale to the photospheric one, see Sect. 3.1.

It should be noted that Pehlivan Rhodin et al. (2021) recently computed new $g f$-values for a large number of $\mathrm{Si}$ I and $\mathrm{Si}$ II lines, which are partly validated against experimental data. Had we adopted those for the seven Si lines in common with our study, the photospheric abundance would have increased to $\log \epsilon_{\mathrm{Si}}=7.53 \pm 0.03$ with a slightly reduced statistical scatter and a corresponding adjustment of the meteoritic abundances. We did, however, opt to retain the older experimental transition probabilities (Garz 1973), renormalised to the lifetimes of Becker et al. (1980) and O'Brian \& Lawler (1991), mainly since the new theoretical values imply a significant ionisation imbalance of 0.1 dex between Si I and Si II, even in 3D non-LTE. The overall agreement with the meteoritic abundances further supports the Garz (1973) data (see Sect. 3.2). Further theoretical and experimental work on Si would be very welcome.

Phosphorus $(Z=15)$. We adopted the results from the $3 \mathrm{D}$ LTE analysis of Scott et al. (2015b): $\log \epsilon_{\mathrm{P}}=5.41 \pm 0.03$. To our knowledge, no non-LTE investigation of $\mathrm{P}$ exists in the literature, neither in 1D nor 3D. Due to the similarity between the weak PI and S I lines, the latter for which non-LTE investigations do exist (see below), comparably small non-LTE effects could be expected for the two elements. The value is unchanged from Asplund et al. (2009).

Sulphur $(Z=16)$. The solar $S$ abundance $\log \epsilon_{\mathrm{S}}=7.12 \pm$ 0.03 is taken from Scott et al. (2015b) who included 1D non-LTE corrections $(-0.01$ dex for optical lines but $-0.05 . .-0.10$ for the IR lines) from Takeda et al. (2005) to their 3D LTE results. The value is unchanged from Asplund et al. (2009).

Potassium $(Z=19)$. The recommended solar $\mathrm{K}$ abundance in Asplund et al. (2009) was $\log \epsilon_{\mathrm{K}}=5.03 \pm 0.09$, which Scott et al. (2015b) updated to $\log \epsilon_{\mathrm{K}}=5.04 \pm 0.05$. The latter result was based on a 3D LTE analysis of five weak lines (excluding the strong 766.5 and $769.9 \mathrm{~nm} \mathrm{~K}$ I resonance lines) together with 1D non-LTE abundance corrections taken from Takeda et al. (1996) in the absence of consistent non-LTE calculations with a 3D solar model. Reggiani et al. (2019) recently presented a new 1D nonLTE analysis with a comprehensive model atom consisting of 133 atomic levels of $\mathrm{K}$ I plus the ground state of $\mathrm{K} \mathrm{II}$ and 250 radiative bound-bound transitions. Particular attention was placed on calculating or adopting reliable cross-sections for inelastic collisions, both with electrons, and with neutral hydrogen. Their 1D non-LTE calculations with a theoretical hydrostatic MARCS model atmosphere using the equivalent widths of three lines (including
K I 769.9nm) yielded a solar abundance of $\log \epsilon_{\mathrm{K}}=5.11 \pm 0.01$ (standard deviation only).

Here, we present a full 3D non-LTE calculations for $\mathrm{KI}$ using the same model atom as in Reggiani et al. (2019). A summary of the inferred abundances for the different lines using a variety of model atmospheres is provided in Table A.1. Compared to Scott et al. (2015b), the $g f$-values have been updated to those recommended by NIST (Kramida 2019), which in all cases are estimated to have an accuracy better than $1 \%$. Furthermore, the spectral line profiles were re-examined and the equivalent widths remeasured, leading us ultimately to exclude the 404.4 and $580.2 \mathrm{~nm}$ lines due to their substantial and uncertain blending. Our recommended solar $\mathrm{K}$ abundance is thus based on the measured equivalent widths of three K I lines: 693.9, 1177.0, and $1252.2 \mathrm{~nm}$. Although we did not consider the K I $769.9 \mathrm{~nm}$ resonance line for the final result due to its large line strength and very pronounced non-LTE effects $(\approx+0.2 \mathrm{dex})$, we note that its 3D non-LTE-based solar $\mathrm{K}$ abundance is fully consistent with those of the weak KI lines. Nevertheless, the departures from LTE are noticeable for the three retained K I lines (3D non-LTE $-3 \mathrm{D}$ LTE $\approx-0.05 \mathrm{dex}$ ), which reinforces the importance of a consistent 3D non-LTE analysis as employed here. Our recommended solar $\mathrm{K}$ abundance, based on the unweighted mean of the three K $\mathrm{I}$ lines, is $\log \epsilon_{\mathrm{K}}=5.07 \pm 0.01$ (statistical) \pm 0.03 (systematic).

Calcium $(Z=20)$. Scott et al. (2015b) slightly revised the present-day photospheric Ca abundance of Asplund et al. (2009) to $\log \epsilon_{\mathrm{Ca}}=6.32 \pm 0.03$ with excellent agreement between the eleven $\mathrm{Ca}$ I and five $\mathrm{Ca}$ II lines employed. These results were based on 3D LTE line formation calculations with our own 1D non-LTE abundance corrections calculated on the $\langle 3 \mathrm{D}\rangle$ model atmosphere, which range from +0.02 to -0.03 dex for $\mathrm{Ca}$ I, and 0.00 to -0.07 dex for Ca II. Recently, Mashonkina et al. (2017) and Osorio et al. (2019) presented new 1D non-LTE investigations that predict more severe, negative, non-LTE corrections for both species but more so for Ca II, which would worsen the ionisation balance obtained in Scott et al. (2015b).

In light of these issues, we carried out a detailed reanalysis of $\mathrm{Ca}$, based on a consistent 3D non-LTE line formation study for the Sun. Our new $\mathrm{Ca}$ model atom consists of $141 \mathrm{Ca}$ I levels, $46 \mathrm{Ca}$ II levels, and the Ca III ground state, which are coupled radiatively by 2271 bound-bound and 135 bound-free transitions. We employed data for inelastic collisions with electrons from Zatsarinny et al. (2019b) for Ca I and from Meléndez et al. (2007) for Ca II; while for inelastic collisions with neutral hydrogen we draw on data from Barklem (2016b) and Belyaev et al. (2018) for Ca I and Ca II, respectively. Osorio et al. (2020) reported a non-negligible influence of non-LTE effects on MgI influencing the statistical equilibrium of $\mathrm{Ca}$ in the Sun. We therefore ran test calculations with the background $\mathrm{Mg}$ opacity treated in non-LTE; however, these were found to have a negligible impact on our results, at least for the $\mathrm{Ca}$ lines adopted in our abundance analysis.

A summary of the inferred abundances for the different lines using a variety of model atmospheres is provided in Table A.1. Compared to Scott et al. (2015b), we adopted the same line data and mostly the same lines, dropping only the $616.13 \mathrm{~nm}$ that is affected by the broad damping wings of the neighbouring $\mathrm{Ca}$ I $616.22 \mathrm{~nm}$ line. The unweighted mean results from the ten $\mathrm{Ca}$ I lines and the five $\mathrm{Ca}$ II lines agree to better than $0.005 \mathrm{dex}$, with a very small line-to-line scatter for each species. Our recommended solar $\mathrm{Ca}$ abundance is $\log \epsilon_{\mathrm{Ca}}=6.30 \pm 0.03$ (total). 


\subsection{Fe-peak elements: Scandium to nickel}

Scandium $(Z=21)$. Scott et al. (2015a) relied on a 3D LTE analysis of five Sc I and nine Sc II lines coupled with nonLTE corrections computed with 1D model atmospheres to determine a solar Sc abundance of $\log \epsilon_{\mathrm{Sc}}=3.16 \pm 0.04$. With the new, highly accurate experimental transition probabilities of Lawler et al. (2019), the weighted average becomes $\log \epsilon_{\mathrm{Sc}}=$ $3.14 \pm 0.01$ (statistical) \pm 0.04 (systematic) $^{6}$. It is noted that the departures from LTE are substantial for the Sc I lines, but the weighted mean Sc abundance is largely driven by Sc II. There is good agreement between the two ionisation stages, both of which give noticeably higher abundances than the CI meteoritic value (Sect. 3.1). Lawler et al. (2019) considered roughly twice as many Sc lines in their solar analysis, many of which are clean and have excellent atomic data; we postpone for later a study of these lines in conjunction with 3D non-LTE calculations and realistic collisional cross-sections.

Titanium $(Z=22)$. In their analysis of Fe-peak elements, Scott et al. (2015a) already benefited from the highly accurate experimental transition probabilities for Ti from the Wisconsin atomic physics group (Ti I: Lawler et al. 2013; Ti II: Wood et al. $2013^{7}$ ). They also applied non-LTE abundance corrections calculated with $1 \mathrm{D}$ model atmospheres, which are significant for Ti I $(\approx+0.06 \mathrm{dex})$ but unimportant for Ti II. Scott et al. (2015a) found a large and unexplained difference in $\mathrm{Ti}$ abundance from the two ionisation stages: $4.88 \pm 0.05$ versus $4.97 \pm 0.04$ from Ti I and Ti II, respectively; their recommended solar Ti abundance was a weighted average of the two, resulting in $\log \epsilon_{\mathrm{Ti}}=$ $4.93 \pm 0.04$. It is noteworthy that all of the considered theoretical and semi-empirical 1D model atmospheres implied significantly larger TiI abundances than with the 3D Stagger model and more in line with the Ti II results. This strong model atmosphere sensitivity suggests that the adopted 1D non-LTE corrections are not appropriate in 3D and that a consistent 3D non-LTE analysis would yield higher Ti I abundances. In contrast to Scott et al. (2015a), we based our recommended solar Ti abundance solely on Ti II but increased the final error somewhat to reflect the discrepancy between the ionisation stages: $\log \epsilon_{\mathrm{Ti}}=4.97 \pm 0.05$ (total). A full 3D non-LTE study with realistic collisional crosssections would be highly desirable; Lawler et al. (2013) and Wood et al. (2013) list a large number of high-quality Ti lines with now excellent atomic data available.

Vanadium $(Z=23)$. The recommended solar photospheric $\mathrm{V}$ abundance of Scott et al. (2015a) was based on a 3D LTE analysis of V I lines with an ad hoc non-LTE correction of 0.10 dex across the board in the absence of actual statistical equilibrium calculations based on the behaviour of Sc and Ti in 1D model atmospheres. As a minority species, V I is very sensitive to the atmospheric structure: the four considered theoretical and semiempirical 1D model atmospheres imply a 0.07-0.18 dex higher abundance. Unfortunately, little guidance is available from the

\footnotetext{
6 Pehlivan Rhodin et al. (2017a) also measured new lifetimes, which coupled with theoretical branching factors yield new rescaled semiempirical Sc II oscillator strengths. Adopting those instead and excluding the highly discrepant $442.07 \mathrm{~nm}$ line would yield a Sc abundance of $\log \epsilon_{\mathrm{Sc}}=3.10 \pm 0.05$. Here, we preferred the arguably less uncertain experimental values of Lawler et al. (2019), which are available for all of our lines.

7 Li et al. (2020) presented a new set of theoretical Ti II $g f$-values, which are in good overall agreement with the Wood et al. (2013) experimental values, although they lead to significantly larger abundance scatter for our lines; we assess the latter source to be more reliable for our purposes.
}

few available V II lines due to their heavy blending, although nominally they suggest $\mathrm{a} \approx 0.1$ dex higher $\mathrm{V}$ abundance, both in 3D and 1D. Updating the Scott et al. (2015a) results with the Lawler et al. (2014) and Wood et al. (2014a) experimental $g f$ values, the mean V I abundance is only +0.01 dex higher, while the V II result changes by $-0.02 \mathrm{dex}^{8}$. We caution that our ad hoc VI non-LTE correction may well be underestimated, and we strongly encourage the first non-LTE investigation of this element to be performed. In the meantime we recommend the value found by Scott et al. (2015a) updated with the Lawler et al. (2014) transition probabilities: $\log \epsilon_{\mathrm{V}}=3.90 \pm 0.08$ (total).

Chromium $(Z=24)$. The solar photospheric $\mathrm{Cr}$ abundance of Scott et al. (2015a) was based on the weighted average of a large number of $\mathrm{Cr}$ I and $\mathrm{Cr}$ II lines with 1D non-LTE abundance corrections. Updating this result with the new experimental Cr II transition probabilities of Lawler et al. (2017) leads to the same mean abundance: $\log \epsilon_{\mathrm{Cr}}=5.62 \pm 0.04$.

Manganese $(Z=25)$. A new solar $M n$ abundance analysis was presented by Bergemann et al. (2019) based on full 3D non-LTE calculations. Using the same 3D hydrodynamical solar model (Magic et al. 2013) as for other elements and equipped with new ab initio inelastic Mn I+H I collisional cross-sections from Belyaev \& Voronov (2017) (see also Grumer \& Barklem 2020), they obtained $\log \epsilon_{\mathrm{Mn}}=5.52 \pm 0.04$. This is $0.10 \mathrm{dex}$ larger than the result of Scott et al. (2015a)(5.42 \pm 0.04$)$ based on 3D LTE model spectra combined with $\langle 3 \mathrm{D}\rangle$ non-LTE abundance corrections; the two results do not overlap within the error bars. The two studies have very few Mn I lines in common, making a detailed comparison difficult. The abundance difference cannot be traced to the adopted transition probabilities since the same sources are employed (mostly Den Hartog et al. 2011 and Blackwell-Whitehead \& Bergemann 2007). Departures from LTE are obviously very prominent, with the 1D non-LTE - 1D LTE difference being +0.07 dex. according to Bergemann et al. (2019). One may suspect that the 3D non-LTE effects and Mn abundances in Bergemann et al. (2019) have been somewhat overestimated due to incomplete UV line blanketing over-estimating the photo-ionisation rates and thus weakening the predicted Mn I lines; a detailed comparison is further complicated by Bergemann et al. (2019) employing different non-LTE radiative transfer codes for their $1 \mathrm{D}$ and $3 \mathrm{D}$ analyses.

Until the situation has been clarified, we advocate the recommended photospheric Mn abundance of Scott et al. (2015a) but with increased errors to reflect the remaining uncertainties associated with the non-LTE modelling: $\log \epsilon_{\mathrm{Mn}}=5.42 \pm 0.06$. Some confidence in this result can be had by noting that Scott et al. (2015a) obtained a value of 5.43 from their consistent $\langle 3 \mathrm{D}\rangle$ nonLTE analysis, only 0.01 dex larger. Our expectation is that, at least for atomic and ionic species in the disc-centre intensity spectrum of the Sun, the full 3D non-LTE result for Mn should be similar to the $\langle 3 \mathrm{D}\rangle$ non-LTE abundance, as we have found in our investigations of $\mathrm{Li}, \mathrm{C}, \mathrm{N}, \mathrm{O}, \mathrm{Na}, \mathrm{Mg}, \mathrm{Al}, \mathrm{Si}, \mathrm{K}, \mathrm{Ca}$, and $\mathrm{Fe}$. Nevertheless, we encourage the issue to be revisited with new 3D non-LTE calculations with realistic inclusion of blending lines both for the statistical equilibrium and for the Mn I abundance diagnostic lines.

\footnotetext{
8 Holmes et al. (2016) measured new experimental transitional probabilities but only for a subset of our solar diagnostics lines. On average, the difference with the Lawler et al. (2014) values is small, $\approx+0.02$ dex, and the line-to-line abundance scatter would increase somewhat. We argue that the Lawler et al. (2014) data are preferable for our purposes (see also Saloman \& Kramida 2017).
} 
Iron $(Z=26)$. In astronomy, $\mathrm{Fe}$ is often used as a proxy for the overall metallicity of a star on account of the element's relatively high abundance and the wealth of readily measurable lines. Iron is also an important opacity contributor in stellar interiors. In the Sun, many lines of both Fe I and Fe II with accurate experimental atomic data are available for a spectroscopic analysis (e.g., Grevesse \& Sauval 1999; Asplund et al. 2000c; Caffau et al. 2011; Mashonkina et al. 2011). From a 3D analysis, Scott et al. (2015a) obtained $7.45 \pm 0.04$ (including 1D nonLTE corrections of $\approx+0.01 \mathrm{dex}$ ) and $7.51 \pm 0.04$, from Fe I and Fe II lines, respectively, leading to a weighted average of $\log \epsilon_{\mathrm{Fe}}=7.47 \pm 0.04$ (statistical + systematic). Lind et al. (2017) updated this to $\log \epsilon_{\mathrm{Fe}}=7.47 \pm 0.04$ based on fully consistent 3D non-LTE calculations for Fe that for the first time were based on a large model atom that included ab initio collisional crosssections for Fe I+H I (Barklem 2018).

We undertook a new analysis of Fe I and Fe II using full 3D non-LTE calculations and improved atomic data. Our new Fe model atom consists of 100 Fe I levels, 76 Fe II levels, and the Fe III ground state, which are radiatively coupled through 4313 bound-bound and 100 bound-free transitions, amounting to 46000 wavelength points. We employed data for inelastic collisions with electrons from Wang et al. (2018) for Fe I and from Bautista et al. (2015) and Zhang \& Pradhan (1995) for Fe II; while for inelastic collisions with neutral hydrogen, we drew on data from Barklem (2018) and Yakovleva et al. (2019) for Fe I and Fe II, respectively. Photoionisation cross-sections for Fe I were sourced from Zatsarinny et al. (2019a). Recently, new accurate experimental transition probabilities for additional excellent solar Fe I lines have been published (Den Hartog et al. 2014; Belmonte et al. 2017), which we employed together with previous lines used by Scott et al. (2015a) with the transition probabilities recommended by the Oxford (e.g., Blackwell et al. 1995), Hannover (e.g., Holweger et al. 1991), and Wisconsin (e.g., O’Brian et al. 1991) groups ${ }^{9}$.

A summary of the inferred abundances for the different lines using a variety of model atmospheres is provided in Table A.2. We now obtain excellent agreement between the two ionisation stages: $7.46 \pm 0.04$ and $7.47 \pm 0.04$ from Fe I and Fe II respectively, based on unweighted means and including statistical and systematic uncertainties. Departures from LTE are small for both species in the Sun. There are no significant dependencies with wavelength, excitation potential, line strength or provenance of the $g f$-values in $3 \mathrm{D}$, in contrast to the results using the 1D semi-empirical Holweger \& Müller (1974) model atmosphere (Fig. 2). With 1D theoretical models, the mean Fe abundance becomes unrealistically $\operatorname{low}\left(\log \epsilon_{\mathrm{Fe}}<7.40\right)$, while with the $\langle 3 \mathrm{D}\rangle$ model, ionisation balance is not fulfilled, thus attesting to the need for full 3D non-LTE calculations. Our recommended photospheric Fe abundance is the average of the Fe I and Fe II results: $\log \epsilon_{\mathrm{Fe}}=7.46 \pm 0.04$ (total), a value which has hardly moved over the past two decades in spite of major improvements in 3D and non-LTE analysis techniques and better atomic data (Asplund et al. 2000c, 2005a, 2009; Bergemann et al. 2012; Scott et al. 2015a; Amarsi et al. 2016b; Lind et al. 2017).

Cobalt $(Z=27)$. We update the Scott et al. (2015a) results with the $g f$-values from Lawler et al. (2015) for the Co I lines in common: $\log \epsilon_{\mathrm{Co}}=4.94 \pm 0.05$ (total). While the new Co abundance is only 0.01 dex higher, the reduced line-to-line scat-

\footnotetext{
9 We did not utilise any of the IR Fe I lines for which new experimental data are now available (Ruffoni et al. 2013) since these particular lines are typically too strong and/or blended in the Sun for a high-precision analysis.
}
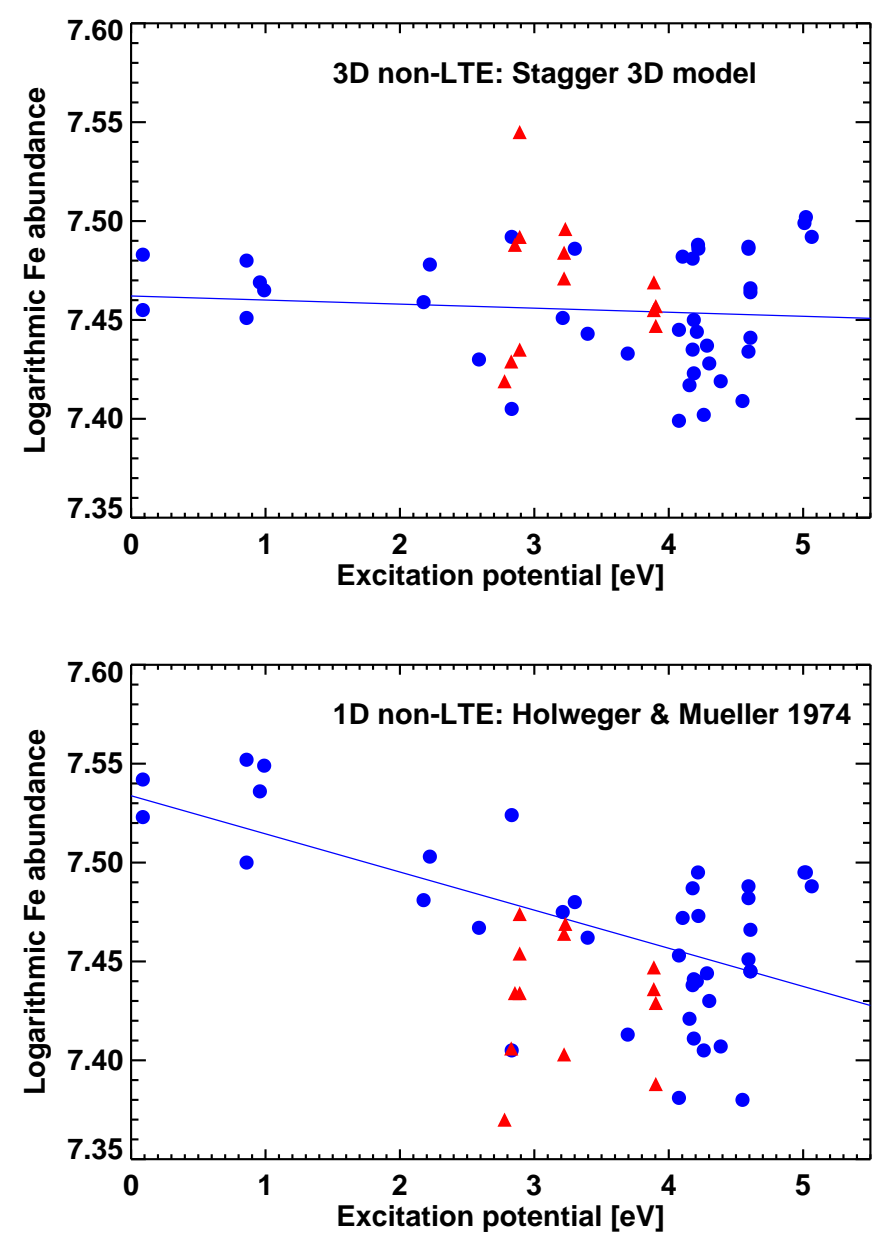

Fig. 2. Inferred photospheric Fe abundances from detailed non-LTE calculations using the 3D hydrodynamical Stagger model (upper panel) and the 1D Holweger \& Müller (1974) model (lower panel). The results for Fe I and Fe II lines are shown as blue circles and red triangles, respectively. The solid lines denote the least-square fits to the $\mathrm{Fe}$ I results. In $3 \mathrm{D}$ non-LTE, there is excellent excitation and ionisation balance for Fe, contrary to the 1D case; it is noted that the trend with excitation potential with the Holweger \& Müller (1974) model is even steeper in LTE (see Table A.2).

ter is a testament to the high accuracy of the new experimental data. As for most Fe-peak elements, one concern relates to the adopted non-LTE corrections, which may be prone to error as they stem from our 1D calculations, and mainly draw on classical and semi-empirical recipes for inelastic collisions with electrons and with neutral hydrogen.

Nickel $(Z=28)$. In the absence of any non-LTE calculations for Ni I, we adopt the 3D LTE based Ni abundance of Scott et al. (2015a), who used the accurate experimental transition probabilities from Wood et al. (2014b): $\log \epsilon_{\mathrm{Ni}}=6.20 \pm 0.04$. Based on our detailed 3D non-LTE calculations for Fe I, we expect the non-LTE effects for Ni I to be similarly minor, but this requires confirmation.

\subsection{The heavy elements: Copper to uranium}

Relatively little work has been done recently to update the solar photospheric analysis by Grevesse et al. (2015) of the neutron capture elements. With the exception of the elements listed below, we adopt their recommended solar abundances. We note 
that many of these elements have few available spectral lines located in the crowded UV region, causing major problems in fitting the lines. Taking an even more conservative approach, we deemed some lines used by Grevesse et al. (2015) as too uncertain for a meaningful abundance determination even if very few, if any, alternative lines of the element are available. In some cases, we increased the error bars to attest to the challenges in working with heavily blended lines; often the blending lines are not even identified. The reader is furthermore cautioned that departures from LTE are largely unexplored territory for the heavy elements in the Sun. This deficiency can be expected to introduce significant systematic errors, quite possible at the level of \pm 0.1 dex, in particular for many of the minority species.

Copper $(Z=29)$. We retain the solar $\mathrm{Cu}$ abundance $\log \epsilon_{\mathrm{Cu}}=$ $4.18 \pm 0.05$ of Grevesse et al. (2015) despite it relying on old and relatively uncertain experimental oscillator strengths (e.g., Kock \& Richter 1968) since the newer theoretical $g f$-values of Liu et al. (2014) imply an unacceptably large line-to-line abundance scatter. New laboratory data for this astrophysically important element would be welcome.

Germanium $(Z=32)$. We reinspected the spectral line profile of the quite strong but blended Ge I $326.95 \mathrm{~nm}$ line and revised the equivalent width downwards relative to Grevesse et al. (2015) to $4.20 \mathrm{pm}$. Our 3D LTE result consequently shifts downwards to $\log \epsilon_{\mathrm{Ge}}=3.62 \pm 0.10$.

Rubidium $(Z=37)$. The only two available $\mathrm{Rb}$ I lines in the solar spectrum, the resonance lines at 780.03 and $794.76 \mathrm{~nm}$, are unfortunately both perturbed by other species. We revised the measured line strengths of Grevesse et al. (2015) (to 0.53 and $0.29 \mathrm{pm}$, respectively) to better account for these blends and adjusted the remaining uncertainties to reflect the difficulties in fitting the weak RbI lines. The resulting 3D LTE abundance is slightly lower than before: $\log \epsilon_{\mathrm{Rb}}=2.44 \pm 0.08$ (total). Very recently, Korotin (2020) performed 1D non-LTE calculations for the $\mathrm{Rb}$ I resonance lines, employing ab initio data for inelastic collisions with neutral hydrogen (Yakovleva et al. 2018). They found substantial departures from LTE $\left(\Delta \log \epsilon_{\mathrm{Rb}}=-0.12\right.$ dex $)$ and a solar $\mathrm{Rb}$ abundance consistent with the meteoritic value for the first time ${ }^{10}$. Their study employed blending lines in the statistical equilibrium computations as well as Rb spectrum synthesis. Applying the new non-LTE corrections of Korotin (2020) to our 3D LTE value above implies a solar $\mathrm{Rb}$ abundance of $\log \epsilon_{\mathrm{Rb}}=2.32 \pm 0.08$.

Rhodium $(Z=45)$. We updated the Grevesse et al. (2015) $\mathrm{Rh}$ abundance with the new experimental transition probabilities for Rh I lines from Malcheva et al. (2015), while restricting the analysis to the $369.24 \mathrm{~nm}$ line due to the heavy blending of the $343.49 \mathrm{~nm}$ line. When also considering the uncertainties in the line strength and $g f$-values, we obtain $\log \epsilon_{\mathrm{Rh}}=0.78 \pm 0.11$ (statistical + systematic). The derived photospheric $\mathrm{Rh}$ abundance remains substantially below the value measured in CI chondrites, which may indicate pronounced departures from LTE for this minority species in the 3D model that are not yet possible to account for. It is noted that $1 \mathrm{D}$ semi-empirical solar

\footnotetext{
${ }^{10}$ Even more recently, Takeda (2021) performed a similar 1D non-LTE study, including for the Sun, where they found a less noticeable nonLTE correction: -0.05 dex. Because they only considered the classical Drawin (1968) formula with a scaling factor for the inelastic $\mathrm{H}$ collisions and no specification is given as to which recipe for the electron collisions is employed, we here give a preference to the results of Korotin (2020). Revisiting the solar Rb abundance issue with 3D nonLTE calculations would be desirable.
}

atmosphere models yield abundances in better agreement with the meteoritic evidence; the shallower temperature gradient of the Holweger \& Müller (1974) model tends to result in smaller departures from LTE (Asplund 2005), a phenomenon termed 'non-LTE masking' by Rutten \& Kostik (1982).

Palladium $(Z=46)$. We reassessed the Pd I lines at 324.27 and $340.46 \mathrm{~nm}$ and revised their equivalent widths upwards relative to Grevesse et al. (2015), to $2.50 \mathrm{pm}$ and $3.58 \mathrm{pm}$, respectively. Our 3D LTE result consequently shifts upwards to $\log \epsilon_{\mathrm{Pd}}=1.57 \pm 0.10$, which is incidentally identical to the value recommended by Asplund et al. (2009).

Barium $(Z=56)$. Unfortunately, only a few spectral lines of $\mathrm{Ba}$ are available as abundance diagnostics, which furthermore are strong and saturated. The solar $\mathrm{Ba}$ abundances presented in Asplund et al. (2009) and Grevesse et al. (2015) were based on 3D LTE calculations to which 1D non-LTE abundance corrections were applied; naturally this approach is not fully selfconsistent, but it was the only one available at the time for this element. Very recently, Gallagher et al. (2020) revisited the issue using full 3D non-LTE computations with the same Stagger 3D hydrodynamical solar model as employed elsewhere in our study. Their inferred solar $\mathrm{Ba}$ abundance of $\log \epsilon_{\mathrm{Ba}}=$ $2.27 \pm 0.01$ (statistical) \pm 0.02 (systematic) was obtained from a 1D LTE spectrum synthesis with a MARCS model atmosphere (Gustafsson et al. 2008) to which abundance corrections from blending lines and 3D (i.e. the differences in abundances in 3D non-LTE to obtain the 1D LTE equivalent width) were applied separately; this is not fully consistent either, but it is a more realistic approach than that in Grevesse et al. (2015). In addition to the three BaII lines employed by Grevesse et al. (2015), Gallagher et al. (2020) also considered the heavily blended $614.17 \mathrm{~nm}$ line and obtained consistent results. It is noted that the 3D LTE abundances in the two studies differ substantially for unknown reasons.

Here, we adopt the Gallagher et al. (2020) 3D non-LTE based result but inflate their uncertainties slightly to account for possible systematic errors from the model atmosphere, non-LTE calculations, and their assumed microturbulence in 1D: $\log \epsilon_{\mathrm{Ba}}=$ $2.27 \pm 0.05$ (total). A new study directly comparing the $3 \mathrm{D}$ nonLTE line profiles (including blends) with observations would be welcome for this important element, especially given the difference with the CI chondritic abundance $\left(\log \epsilon_{\mathrm{Ba}}=2.18 \pm 0.02\right)$.

Praseodymium $(Z=59)$. We apply the $1 \mathrm{D}$ non-LTE abundance correction $(+0.03 \mathrm{dex})$ for Pr I from Mashonkina et al. (2009) to the 3D LTE results of Grevesse et al. (2015), which in turn was based on 3D abundance corrections applied to the 1D spectrum synthesis of Sneden et al. (2009). The revised Pr abundance becomes $\log \epsilon_{\mathrm{Pr}}=0.75 \pm 0.05$.

Tungsten $(Z=74)$. Compared to Grevesse et al. (2015), we remove the weak $484.38 \mathrm{~nm}$ line owing to uncertainties in measuring its equivalent width. Therefore, restricting the 3D LTE analysis of Grevesse et al. (2015) to the W I $400.875 \mathrm{~nm}$ line implies $\log \epsilon_{\mathrm{W}}=0.79 \pm 0.11$.

Osmium $(Z=76)$. After inspecting the spectral line profile of the Os I $330.16 \mathrm{~nm}$ transition, we revise its equivalent width downwards relative to Grevesse et al. (2015), to $0.86 \mathrm{pm}$. The resulting 3D LTE result is 0.05 dex lower: $\log \epsilon_{\mathrm{Os}}=1.35 \pm 0.12$.

Iridium $(Z=77)$. We judge that the only detectable Ir I line, at $322.08 \mathrm{~nm}$, is too badly blended and has too uncertain a continuum placement to allow us to make a meaningful solar Ir abundance measurement. 
Gold $(Z=79)$. Accounting for the uncertain continuum placement around the weak and badly blended Au I $312.28 \mathrm{~nm}$ line leads to a more conservative error estimate than in Grevesse et al. (2015): $\log \epsilon_{\mathrm{Au}}=0.91 \pm 0.12$ (total).

Lead $(Z=82)$. We update the solar $\mathrm{Pb}$ abundance based on our new measurement of the equivalent width $(0.91 \mathrm{pm})$ of the $\mathrm{Pb}$ I $368.35 \mathrm{~nm}$, which results in $\log \epsilon_{\mathrm{Pb}}=1.95 \pm 0.08,0.03 \mathrm{dex}$ larger than in Grevesse et al. (2015).

\subsection{Elements observed in sunspots: Fluorine, chlorine, indium, and thallium}

Fluorine $(Z=9)$. While no spectral lines of $F$ are detectable in the photospheric spectrum of the quiet Sun, there are features of the HF molecule in sunspot spectra that enable a determination of the solar F abundance. Maiorca et al. (2014) analysed the umbral atlas of Wallace et al. (2001) with a 1D theoretical model atmosphere corresponding to an effective temperature of $T_{\text {eff }}=4250 \mathrm{~K}$ to estimate $\log \epsilon_{\mathrm{F}}=4.40 \pm 0.25$; most of the uncertainty stems from the choice of model atmosphere since the employed radiative-equilibrium model without magnetic fields may not be appropriate for this particular sunspot umbra. Here, we recommend the value of Maiorca et al. (2014), which represents a 0.16 dex reduction compared to the older Hall \& Noyes (1969) result adopted by Asplund et al. (2009).

Chlorine $(Z=17)$. Similar to $\mathrm{F}$, the photospheric $\mathrm{Cl}$ abundance can only be estimated from sunspot spectra since the $\mathrm{HCl}$ molecule requires cool temperatures; no atomic features of $\mathrm{Cl}$ are present in the solar spectrum. Recently, Maas et al. (2016) revisited the previous $\mathrm{HCl}$ sunspot study of Hall \& Noyes (1972) with improved molecular data and observations to obtain a 0.19 dex lower abundance: $\log \epsilon_{\mathrm{Cl}}=5.31 \pm 0.12$. Given the remaining model challenges, especially in finding a suitable model atmosphere for a sunspot of unknown $T_{\text {eff }}$, we argue that the quoted uncertainty is likely overly optimistic, and we instead adopt \pm 0.20 dex. A more accurate determination of the solar abundances of the halogen elements would be possible through the abundance ratio of another species with similar temperature dependence and spectral features with well-determined $g f$ values, such as $\mathrm{FeH}$ and $\mathrm{MgH}$.

Indium $(Z=49)$. Analyses of the In I $451.13 \mathrm{~nm}$ spectral line in the quiet Sun have traditionally yielded an In abundance much higher than the CI chondritic value (e.g., Bord \& Cowley 2002: $\left.\log \epsilon_{\mathrm{In}}=1.56 \pm 0.20\right)$. Vitas et al. (2008) showed that this is likely due to an unknown blend and instead confirmed that the solar In abundance is close to the meteoritic value based on a sunspot spectrum: $\log \epsilon_{\mathrm{In}}=0.80 \pm 0.20$. We adopt this value here, as we did in Asplund et al. (2009).

Thallium $(Z=81)$. The most recent determination of the solar $\mathrm{Tl}$ abundance dates back almost half a century. Updating with the current NIST-recommended transition probability for the only viable but heavily blended Tl I $535.04 \mathrm{~nm}$ line, the sunspot analysis of Lambert et al. (1972) suggests $\log \epsilon_{\mathrm{Tl}}=$ $0.92 \pm 0.17$, quite likely with an underestimated uncertainty. A more accurate determination would be possible from the abundance ratio with another similar species such as $\mathrm{Zr}$ I.

\subsection{Indirect abundance determinations: Noble elements}

The photospheric abundance of the noble gases cannot be inferred directly from spectroscopy due to the extremely high excitation potentials and thus minuscule level populations involved. Other indirect methods are therefore required.

Helium $(Z=2)$. The solar He abundance can be derived from the corona, prominences, solar wind, solar-energetic particles, and the gas giant planets. However, the $\mathrm{He} / \mathrm{H}$ ratio varies greatly depending on environment, from 0.01 to 0.09 (e.g., Bochsler 2007; Reames 2014). The Genesis sample return mission of the solar wind implies a ratio of about 0.05 (Huss et al. 2020), which is significantly lower than expected in the Sun as a result of Coulomb-drag fractionation.

Here, we instead rely on helioseismology, which enables an accurate determination of the He abundance in the solar convection zone and thus photosphere, which cannot be probed spectroscopically. The change in the adiabatic index $\Gamma_{1}=$ $(\partial \ln P / \partial \ln \rho)_{\mathrm{S}}$ in the He II ionisation zone occurring at temperatures $T \approx 10^{5} \mathrm{~K}$ (corresponding to a radius of $r \approx 0.98 R_{\odot}$ ) leaves an observable signature in the $p$-mode oscillation frequencies that depends on the He content (e.g., Basu 2016 and references therein). In Asplund et al. (2009), we adopted the helioseismic He mass fraction value of $Y_{\text {surface }}=0.2485 \pm 0.0034$ from Basu \& Antia (2004); most of the quoted uncertainty stems from the choice of equation of state (OPAL: Rogers \& Nayfonov 2002 vs. MHD: Mihalas et al. 1988). Due to the overlapping higher ionisation zones of other abundant elements, especially $\mathrm{O}$, there is a degeneracy with the adopted metal mass fraction $Z_{\text {surface }}$ in the reference solar model for the helioseismic inversion: $\delta Y_{\text {surface }} / \delta Z_{\text {surface }}=-1.06$ and -0.56 with the OPAL (Basu \& Antia 2004) and MHD (Trampedach et al. 2006) equation of states, respectively. Using a similar technique but with the improved SAHA-S3 equation of state, Vorontsov et al. (2013) obtained maximum-likelihood estimates of $Y_{\text {surface }}=$ $0.240-0.255$ and $Z_{\text {surface }}=0.008-0.013$.

Taking the mean of the Basu \& Antia (2004) and Vorontsov et al. (2013) He abundances corrected to our recommended present-day photospheric composition $\left(Z_{\text {surface }}=\right.$ 0.0139 , Sect. 5) implies $Y_{\text {surface }}=0.2423 \pm 0.0054$, which we adopt here. In terms of the standard logarithmic scale, this corresponds to $\log \epsilon_{\mathrm{He}}=10.914 \pm 0.013$, or $\mathrm{He} / \mathrm{H}=0.082$ by number density.

Neon $(Z=10)$. The absolute Ne abundance can be determined from the radiation originating in the high-temperature regions of the Sun (e.g., transition region, corona, flares) or in situ measurements of the solar wind and solar energetic particles. Both types of analyses are, however, complicated by the existence of the still poorly understood first ionisation potential (FIP) effect (e.g., Laming 2015); the abundances in the upper solar atmosphere and solar wind are modified relative to the photospheric values by a degree inversely dependent on the elemental ionisation potential. Arguably a more reliable approach, that we employ here, is to combine the abundance ratio of $\mathrm{Ne}$ $\left(\chi_{\text {ion }}=21.56 \mathrm{eV}\right)$ with an element with a similarly high ionisation potential and its photospheric abundance, under the assumption that the two elements experience the same, or at least a very similar, FIP effect.

A common comparison element is $\mathrm{O}\left(\chi_{\text {ion }}=13.62 \mathrm{eV}\right)$ since it can be measured in conjunction with $\mathrm{Ne}$ in the upper atmosphere and solar wind, and its photospheric abundance can be determined spectroscopically (albeit with challenges, Sect. 2.3); it is certainly conceivable that the difference in ionisation potential could still introduce elemental variations. Young (2018) recently revisited the measured abundance ratios in the transition region of the quiet Sun, finding a significantly higher $\mathrm{Ne} / \mathrm{O}$ ratio than previously estimated: $\mathrm{Ne} / \mathrm{O}=0.24 \pm 0.05$ compared with 
$0.175 \pm 0.031$ by Young (2005). Most of this adjustment comes from improvements in the atomic data, resulting in changes to the ionisation and recombination rates. Together with our photospheric $\mathrm{O}$ abundance, this would imply a photospheric Ne abundance of $\log \epsilon_{\mathrm{Ne}}=8.08 \pm 0.09$, a $40 \%$ increase compared with the value recommended in Asplund et al. (2009) using the Young (2005) $\mathrm{Ne} / \mathrm{O}$ ratio.

Landi et al. (2007) inferred the absolute coronal Ne abundance from solar flare spectra: $\log \epsilon_{\mathrm{Ne}}=8.11 \pm 0.12$. There are, however, variations between flares both in the absolute $\mathrm{Ne}$ abundances (e.g., $\log \epsilon_{\mathrm{Ne}}=7.92 \pm 0.03$ Landi \& Testa (2015) and in the $\mathrm{Ne} / \mathrm{O}$ ratio during the solar cycle as a result of the FIP effect. It is expected that the results are the least susceptible to the FIP effect during solar minima, for which Landi \& Testa (2015) obtained $\mathrm{Ne} / \mathrm{O}=0.25 \pm 0.05$, in perfect agreement with the quiet Sun transition region determination by Young (2018). The $\mathrm{Ne} / \mathrm{O}$ ratio is typically lower in solar energetic particles (e.g., 0.140-0.157 \pm 0.010 , Reames 2020) and solar wind (e.g., 0.11-0.14, Reisenfeld et al. 2013), which suggests the existence of additional elemental fractionation. Thus, inferences of the absolute solar composition directly from solar wind measurements (e.g., von Steiger \& Zurbuchen 2016) are likely prone to systematics and are probably unreliable (for further discussion see Serenelli et al. 2016).

An independent probe of the $\mathrm{Ne}$ abundance is offered by the measurements from the Genesis sample return mission (Burnett et al. 2019). Genesis offers a comparison with $\mathrm{He}$, which has a more similar ionisation potential $\left(\chi_{\text {ion }}=24.59 \mathrm{eV}\right)$ to $\mathrm{Ne}$ than $\mathrm{O}$ has. The quoted ${ }^{4} \mathrm{He} /{ }^{20} \mathrm{Ne}$ and ${ }^{20} \mathrm{Ne} /{ }^{22} \mathrm{Ne}$ ratios from the Genesis study of Heber et al. (2009) together with the helioseismic $\mathrm{He} / \mathrm{H}$ ratio in the convection zone lead to a solar $\mathrm{Ne}$ abundance of $\log \epsilon_{\mathrm{Ne}}=8.14 \pm 0.01$. When accounting for correlations in the measured $\mathrm{Ne} / \mathrm{He}$ and $\mathrm{H} / \mathrm{He}$ ratios in different solar wind regimes, the abundance becomes $\log \epsilon_{\mathrm{Ne}}=8.06 \pm 0.03$ (Burnett et al. 2019; Huss et al. 2020), which is in excellent agreement with the above estimate using the Young (2018) Ne/O ratio. In the absence of a proper understanding of the physical processes responsible for the FIP effect, it is difficult to estimate the full uncertainties attached to this Genesis abundance. We tentatively assign half the difference between Heber et al. (2009) and Burnett et al. (2019) as a systematic error, which leads to our final recommended solar photospheric Ne abundance of $\log \epsilon_{\mathrm{Ne}}=8.06 \pm 0.03$ (statistical) \pm 0.04 (systematic). The excellent agreement with the coronal value by Young (2018) lends strong support to this 0.13 dex higher abundance than in Asplund et al. (2009) and consequently also the here advocated photospheric $\mathrm{O}$ abundance.

Argon $(Z=18)$. Asplund et al. (2009) used the straight average from a variety of methods to arrive at a solar Ar abundance of $\log \epsilon_{\mathrm{Ar}}=6.40 \pm 0.13$ following the procedure outlined by Lodders (2008): solar wind, solar flares and energetic particles, nuclear statistical equilibrium, Jupiter, and solar neighbourhood. Updating this exercise with new data and down-weighting the planetary and stellar data would lead to $\log \epsilon_{\mathrm{Ar}}=6.36 \pm 0.11$.

Here, we instead opt for a single, high-precision measurement: the Genesis sample return of the solar wind (Burnett et al. 2019), in the same manner as our recommended $\mathrm{Ne}$ abundance was determined. Allowing for correlations between the measured $\mathrm{H} / \mathrm{He}$ and ${ }^{36} \mathrm{Ar} /{ }^{4} \mathrm{He}$ ratios, Burnett et al. (2019) and Huss et al. (2020) obtained $\log \epsilon_{\mathrm{Ar}}=6.38 \pm 0.08$ (statistical). We estimate that the remaining systematic errors are significant given the still poorly understood FIP effect (Laming 2015) and the differences in ionisation potential between $\operatorname{Ar}\left(\chi_{\text {ion }}=\right.$
$15.76 \mathrm{eV})$ and the reference element $\mathrm{He}\left(\chi_{\text {ion }}=24.59 \mathrm{eV}\right)$; it is noted that Meshik et al. (2020) have argued that the FIP fractionation still varies with ionisation potential even for $\chi_{\text {ion }}>13 \mathrm{eV}$. We adopt $\log \epsilon_{\mathrm{Ar}}=6.38 \pm 0.10$ for the Sun.

Krypton $(Z=36)$. Here, we base the recommended solar $\mathrm{Kr}$ abundance on the Genesis measurements of the solar wind by Meshik et al. (2020). With the quoted $\mathrm{Kr}$ and $\mathrm{H}$ fluences, one obtains $\log \epsilon_{\mathrm{Kr}}=3.12 \pm 0.03$ (statistical). This value is preconditioned on the assumption that the FIP fractionation corrections are identical for $\mathrm{Kr}\left(\chi_{\text {ion }}=14.00 \mathrm{eV}\right)$ and $\mathrm{H}\left(\chi_{\text {ion }}=13.60 \mathrm{eV}\right)$ even when individually the corrections are about a factor of three. Alternatively, using the $\mathrm{Kr}$ and $\mathrm{Ar}\left(\chi_{\text {ion }}=15.76 \mathrm{eV}\right)$ fluences together with the above estimated solar Ar abundance leads to a slightly higher value with a greater statistical uncertainty: $\log \epsilon_{\mathrm{Kr}}=3.16 \pm 0.08$. Burnett et al. (2019) found a $\mathrm{Kr}$ abundance 0.2 dex lower with the same Genesis data when using $\mathrm{O}\left(\chi_{\text {ion }}=13.62 \mathrm{eV}\right)$ as the reference element, which may imply element-specific corrections for high-FIP elements beyond theoretical predictions (Meshik et al. 2020), or that the true photospheric $\mathrm{O}$ abundance is correspondingly much higher than advocated here (Laming et al. 2017). In all likelihood, the uncertainty is dominated by systematic errors from the FIP effect, whichever method is chosen. We adopt $\log \epsilon_{\mathrm{Kr}}=3.12 \pm 0.10$ including estimated systematic errors.

Traditionally, the solar $\mathrm{Kr}$ abundance has been estimated from the smooth variations in the abundances of nearby oddmass number isotopes in CI chondrites (Sect. 3) as predicted by the measured cross-sections for the slow neutron capture process (e.g., Wisshak et al. 1998), a method originally proposed by Suess (1947a,b). With updated isotopic abundances, this leads to $\log \epsilon_{\mathrm{Kr}}=3.15 \pm 0.10$ (statistical). The true uncertainty is likely larger given that the odd-nuclei abundance smoothness has been questioned and that a more realistic distribution of neutron exposures than in this classical model leads to significantly different predictions for $\mathrm{Kr}$ (Arlandini et al. 1999). Nevertheless, it is reassuring that this completely independent method agrees well with the estimate based on the solar-wind.

Xenon $(Z=54)$. The Xe abundance can be inferred via the photospheric Sm measurement combined with experimental neutron capture cross-sections under the assumption that the reaction flow has reached equilibrium. Using their measured cross-sections for the pure s-process isotopes, ${ }^{130} \mathrm{Xe}$ and ${ }^{150} \mathrm{Sm}$ Reifarth et al. (2002) obtained $\log \epsilon_{\mathrm{Xe}}=2.24 \pm 0.01$ (statistical). With our updated photospheric Sm abundance, we revise this estimate slightly to $\log \epsilon_{\mathrm{Xe}}=2.22 \pm 0.05$, including estimated systematic errors (adopting the CI chondritic Sm abundance instead to normalise the $s$-process production would imply a 0.01 dex lower Xe abundance). The solar wind abundance inferred from Genesis data (Meshik et al. 2020) is significantly higher, $\log \epsilon_{\mathrm{Xe}}=2.42 \pm 0.05$ (statistical), suggesting a different FIP fractionation between $\mathrm{Xe}\left(\chi_{\text {ion }}=12.13 \mathrm{eV}\right)$ and $\mathrm{H}$, as also expected on theoretical grounds (Laming et al. 2017).

\section{Comparison with meteoritic abundances}

\subsection{Chemical composition of $\mathrm{Cl}$ chondrites}

Independent and complementary data on the overall chemical composition of the Solar System are offered by meteorites. The vast majority of the more than 50000 meteorites found on Earth have undergone fractionation and alteration to varying degrees, leaving just a handful of the most primitive and undifferentiated carbonaceous chondrites, the so-called CI chondrites: the Alais, 
Ivuna, Orgueil, Revelstoke, and Tonk meteorites, named after the locations of the falls ${ }^{11}$. On account of the overall similarities with the solar photospheric abundances, it has long been argued that the CI chondrites contain pristine samples of the original proto-solar nebula (e.g., Urey 1952, Cameron 1968; see Lodders 2019 for a historical overview). The aqueous alteration and thermal metamorphism of the textural and mineralogical structure all CI chondrites have experienced are not believed to have significantly changed its overall chemical composition, with the exception of the highly volatile elements that are strongly depleted (Palme et al. 2014) ${ }^{12}$. Since these depleted elements are also the most cosmically abundant, the meteoritic abundances by number densities are therefore traditionally normalised relative to Si: $N_{\mathrm{Si}} \equiv 10^{6}$ (Suess \& Urey 1956). To anchor the two sets of abundances onto the same scale, the meteoritic and photospheric Si abundances are therefore set equal with our recommended solar Si abundance (Sect. 2.4): $\log \epsilon_{\mathrm{X}}=1.51+\log N_{\mathrm{X}}$. In principle, more elements can be included in the conversion (e.g., Anders \& Grevesse 1989; Lodders et al. 2009; Palme et al. 2014, who used 12, 39, and 38 elements, respectively), but for simplicity we only employ $\mathrm{Si}$, which was also done by Asplund et al. (2005a, 2009) and Lodders (2019); we would have obtained the same result had we adopted the same normalisation elements as Palme et al. (2014).

Several literature compilations of the chemical compositions of CI chondrites exist, with Wasson \& Kallemeyn (1988), Anders \& Grevesse (1989), Lodders (2003), Lodders et al. (2009), Palme et al. (2014), and Lodders (2019) being particularly highly regarded and authoritative. These studies provide a comprehensive curation of a large number of original references of laboratory data on meteorites. Here (see Table 2), we adopt the CI chondritic abundances recommended by Palme et al. (2014) for the 83 elements for which data from the Orgueil meteorite exist. To convert the meteoritic abundance measurements by mass to the standard meteoritic abundance scale by number, we used the atomic weights recommended by Meija et al. (2016a) with a few adjustments to accommodate more representative Solar System isotopic ratios as described in Sect. 4. The quoted CI abundance uncertainties are only statistical sampling errors.

We did not attempt to factor in the recent measurements by Braukmüller et al. (2018) on account of the surprisingly large variation between their analyses of Orgueil and Ivuna, as well as with prior CI measurements for several elements, including $\mathrm{Na}$, $\mathrm{K}, \mathrm{Zr}$, the rare Earth elements, and Th and U (Fig. 3). The Ivuna analysis by King et al. (2020) agrees well with the Palme et al. (2014) recommended abundances overall, although some striking discrepancies exist, including for $\mathrm{S}, \mathrm{P}, \mathrm{K}, \mathrm{Zr}, \mathrm{Ba}, \mathrm{Hf}$, Ta, $\mathrm{W}$, and Th and $\mathrm{U}$ (Fig. 3). These differences can largely be traced to increased mobility in fluids for some elements during the aqueous alterations of the CI chondrites (Palme et al. 2014; Braukmüller et al. 2018). The recommended values of Palme et al. (2014) agree very well with the newer compilation by Lodders (2019), with a few exceptions. Notable changes have occurred for C, N, F, Br, Sb, I, and Hg, as seen in Fig. 3, but those elements are also among the most uncertain. Lodders (2019) performed a weighted average of the available analyses of all $\mathrm{CI}$

\footnotetext{
${ }^{11}$ Orgueil is the largest CI chondrite weighing in at $14 \mathrm{~kg}$, followed by Alais $(6 \mathrm{~kg})$ and Ivuna $(0.7 \mathrm{~kg})$. In comparison, the Tonk $(8 \mathrm{~g})$ and Revelstoke (1 g) meteorites are tiny, and as a result very few mass spectroscopic analyses have been undertaken on them.

${ }^{12}$ Large CI chondrites such as Orgueil do display some heterogeneity due to parent body redistribution of elements, which contributes significantly to the statistical uncertainties of the meteoritic abundances (Palme et al. 2014).
}

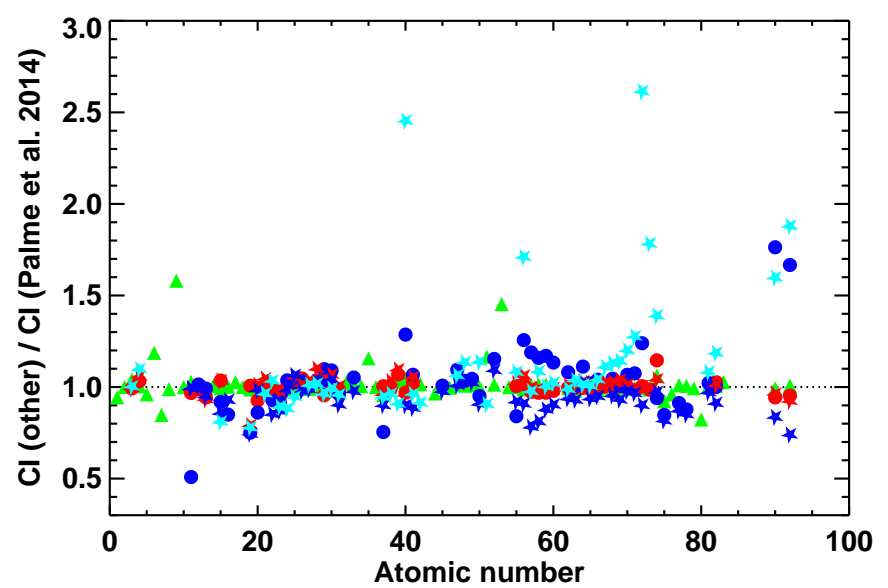

Fig. 3. Comparison between the recommended CI chondritic abundances by Palme et al. (2014), which we have adopted here, with other recent CI analyses (red symbols: Barrat et al. 2012; blue: Braukmüller et al. 2018; turquoise: King et al. 2020; green: Lodders 2019). Circle symbols correspond to data for the Orgueil meteorite, star symbols are for the Ivuna CI chondrite, and triangles for mean of all CI chondrites. All data are published abundances (ppm by mass) relative to Palme et al. (2014).

chondrites, but since they did not provide references to the original data, we instead opted for the compilation of Palme et al. (2014).

\section{2. $\mathrm{Cl}$ chondritic and photospheric abundances}

Figure 4 shows a comparison of our recommended present-day solar photospheric abundances with those from CI chondrites on the astronomical logarithmic abundance scale. Clearly, the agreement is very good overall, with a few striking and wellknown differences. The noble gases ( $\mathrm{He}, \mathrm{Ne}, \mathrm{Ar}, \mathrm{Kr}, \mathrm{Xe}$ ) are highly depleted in CI chondrites by factors ranging from $>10^{4}$ (Xe) to $>10^{9}(\mathrm{He}, \mathrm{Ne})$, while the other highly volatile gases $\mathrm{H}, \mathrm{C}, \mathrm{N}$, and $\mathrm{O}$ are depleted by factors of $10^{4}, 10,40$, and 2 , respectively.

The solar photospheric Li abundance is a factor of almost 200 lower than in CI chondrites due to nuclear processing in the Sun, much more than predicted by standard solar models. It implies additional mixing below the solar convection zone, bringing gas down to high enough temperatures $\left(\approx 2.5 \cdot 10^{6} \mathrm{~K}\right)$ for nuclear destruction to take place and subsequently returning it to the convection zone (e.g., Charbonnel \& Talon 2005). Several possible physical processes for this mixing have been proposed (e.g., internal gravity waves, rotation, convective overshooting, and atomic diffusion or a combination thereof), but all available modelling still requires parametrisation and hence remains uncertain. Solar twins show a slow and monotonically increasing Li depletion with age (Carlos et al. 2019), with the Sun being unusually deficient in $\mathrm{Li}$ by $\approx 0.3$ dex given its age for reasons not yet understood. There is a long and chequered history surrounding the question of whether Li depletion in the Sun and similar stars is related to planet formation (e.g., Bouvier 2008; Israelian et al. 2009; Baumann et al. 2010; Baraffe \& Chabrier 2010).

Given the existence of additional mixing below the convection zone as evident from $\mathrm{Li}$, the photospheric abundances of $\mathrm{Be}$ and $\mathrm{B}$ take on particular importance, since they burn at slightly higher temperatures than $\mathrm{Li}\left(\approx 3.5 \cdot 10^{6} \mathrm{~K}\right.$ and $\approx 5 \cdot 10^{6} \mathrm{~K}$, respectively). The fact that solar twins spanning a wide age 


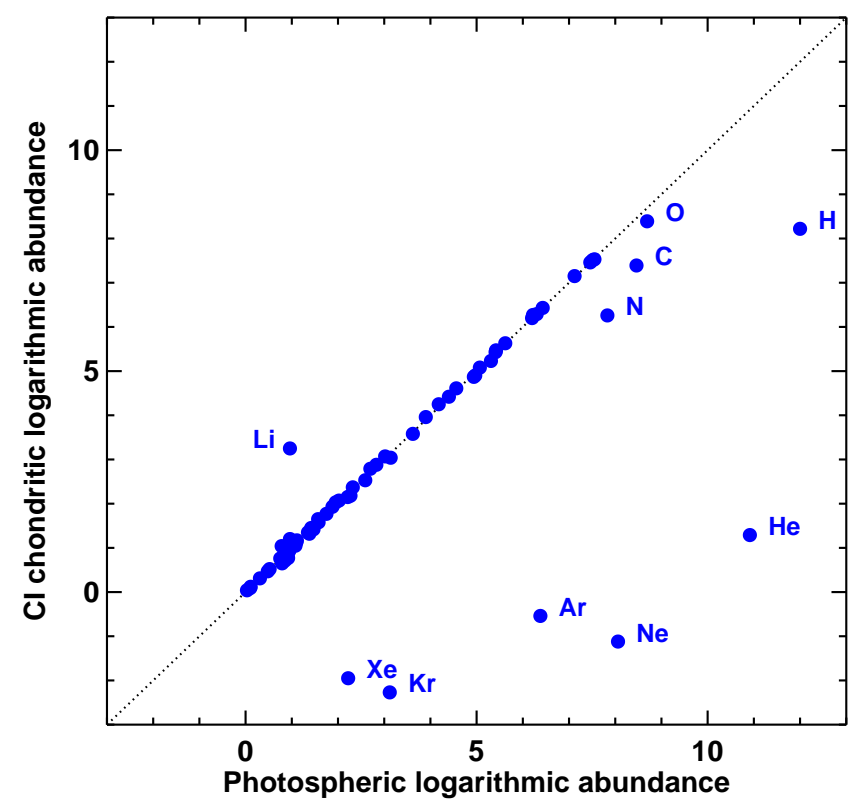

Fig. 4. Comparison between our recommended abundances in presentday solar photosphere and CI chondrites. Most elements agree well, with the exception of the highly volatile elements, which are depleted in meteorites, and $\mathrm{Li}$, which has been largely destroyed in the Sun due to nuclear burning.

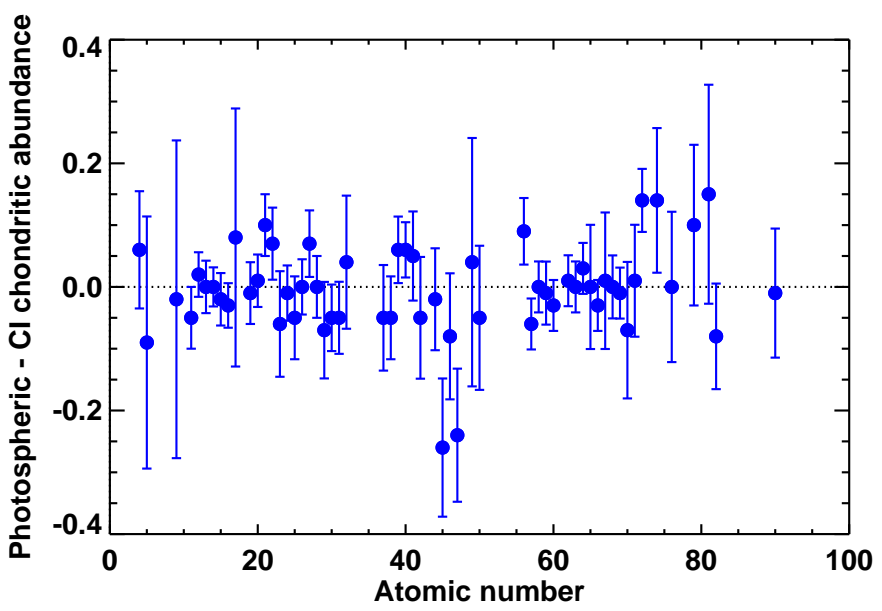

Fig. 5. Differences of the photospheric and CI chondritic logarithmic abundances as functions of atomic number. In most cases, the elemental abundances agree within the combined uncertainties; the highly volatile elements and $\mathrm{Li}$ are not shown in the figure due to meteoritic and solar depletion, respectively. The two most discrepant elements are $\mathrm{Rh}$ and $\mathrm{Ag}$, both of which are suspected to have underestimated photospheric abundances in the absence of non-LTE calculations.

range all have very similar photospheric Be abundances suggests that the Sun has not experienced significant Be depletion in the convection zone, even though the solar Be abundance is perhaps $\approx 0.05$ dex lower than expected for its age (Tucci Maia et al. 2015). A modest B deficiency could, in principle, be hidden within the large error bars, but the absence of a significant $\mathrm{Be}$ depletion argues against it. The photospheric $\mathrm{Li}, \mathrm{Be}$, and $\mathrm{B}$ abundances imply shallow extra mixing below the convection zone present over Gyr timescales in the Sun.

A more detailed comparison of the photospheric and CI chondritic abundances as a function of atomic number is shown in Fig. 5. With the exception of the depleted elements men- tioned above, essentially all elements agree within their combined uncertainties. Using the inverse of the combined photospheric and meteoritic abundance uncertainties in quadrature as weights, the mean average difference of the 58 non-depleted elements (i.e. excluding $\mathrm{Li}$ and the highly volatiles) amounts to $0.000 \pm 0.007$ (s.d. $=0.052$ ) dex. We remind the reader that the meteoritic abundance scale has been normalised to the photospheric $\mathrm{Si}$ abundance, which is here only quoted to within $0.01 \mathrm{dex}$, and hence this perfect agreement is somewhat fortuitous. This comparison does, however, support our choice of transition probabilities for Si (Sect. 2.4). Likewise, it is noted that atomic diffusion and gravitational settling taking place in the Sun over the past $4.57 \mathrm{Gyr}$ will retain this overall agreement since all elements bar $\mathrm{H}$ and $\mathrm{He}$ diffuse in nearly identical amounts (Sect. 5). Of these 58 elements, four are nominal $>2 \sigma$ outliers: Sc, Rh, Ag, and Hf; we suspect that the photospheric abundances from the minority ionisation stages Rh I and $\mathrm{Ag}$ I to have been significantly underestimated in the absence of non-LTE calculations (Sect. 2.6). Overall, CI chondrites closely resemble the solar photospheric abundances, justifying their historical use as a compositional proxy. Caveats are in place, however, as discussed below.

\subsection{Chemical signatures of planet formation}

The overall impressive consistency between the CI chondrites and the solar photosphere notwithstanding, it is worthwhile to ask whether the two abundance sets are truly the same. Or in other words, are CI chondrites a true reflection of the pristine chemical composition of the proto-solar nebula as has long been assumed (e.g. Suess \& Urey 1956)? Figure 6 shows the differences between the photospheric and the CI meteoritic logarithmic abundances as a function of $50 \%$ equilibrium condensation temperature $\left(T_{\text {cond }}\right)$ computed for solar composition gas at a pressure of $10^{-4}$ bar (Wood et al. 2019, see also Lodders 2003); for clarity, only elements with a combined abundance uncertainty of $<0.10$ dex are included. With the anchoring of the two abundance scales through $\mathrm{Si}\left(T_{\text {cond }}=1314 \mathrm{~K}\right)$, it appears as though the moderately volatile elements $\left(300 \lesssim T_{\text {cond }} \lesssim\right.$ $1250 \mathrm{~K}$; here: $\mathrm{Pb}, \mathrm{S}, \mathrm{Zn}, \mathrm{Rb}, \mathrm{K}, \mathrm{Ga}, \mathrm{Cu}, \mathrm{Na}, \mathrm{Mn}$, in order of increasing $T_{\text {cond }}$ ) are enriched in the CI chondrites relative to the Sun: the weighted mean difference is $-0.042 \pm 0.006$ (s.d.= 0.018) dex. Not surprisingly, the main component elements $\left(1250 \lesssim T_{\text {cond }} \lesssim 1370 \mathrm{~K}\right.$; here: $\mathrm{Mg}, \mathrm{P}, \mathrm{Si}, \mathrm{Cr}, \mathrm{V}, \mathrm{Fe}$, $\mathrm{Co}, \mathrm{Ni}$ ) agree excellently, due to the fact that we enforced the $\mathrm{Si}$ abundances to agree: $+0.004 \pm 0.009($ s.d. $=0.025) \mathrm{dex}$. The refractory elements ( $T_{\text {cond }} \gtrsim 1370 \mathrm{~K}$, here 24 elements) may be slightly depleted in CI chondrites, but the relatively large scatter prevents a firm conclusion: $+0.015 \pm 0.010(\mathrm{~s} . \mathrm{d} .=0.048) \mathrm{dex}$. We caution that the absolute level of enhancement/depletion depends on the chosen reference element. It is well known that the CI chondrites are volatile rich compared to the other carbonaceous chondrites, but here we thus find evidence that this is also the case in comparison to the Sun. The opposite is of course true for the highly volatile $\left(T_{\text {cond }} \lesssim 250 \mathrm{~K}\right)$ elements $(\mathrm{H}$, $\mathrm{He}, \mathrm{C}, \mathrm{N}, \mathrm{O}, \mathrm{Ne}, \mathrm{Ar}, \mathrm{Xe}, \mathrm{Kr}$ ), which are greatly depleted in all meteorites, including the CI chondrites (Sect. 3.1). This disposition for volatiles and refractories in the Sun and CI chondrites was first noticed by Gonzalez (1997) when discussing the metalrichness of the first discovered exoplanet host stars, and it is here substantiated by our more accurate photospheric abundances.

There is significant scatter in Fig. 6 around any possible trend, mostly driven by the photospheric abundance uncertainties; higher precision is naturally obtained when instead 


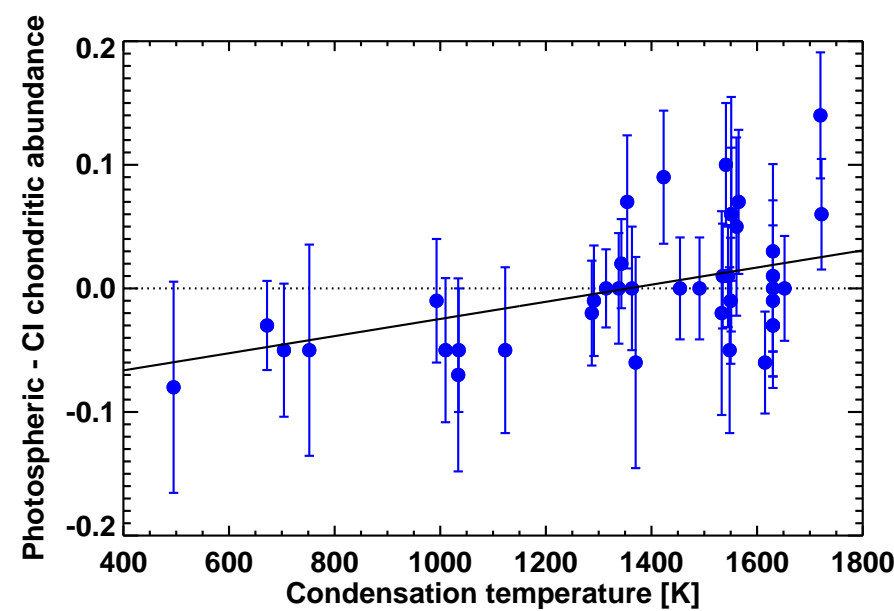

Fig. 6. Differences of the photospheric and CI chondritic logarithmic abundances as a function of $50 \%$ condensation temperature at a pressure of $10^{-4}$ bar for a solar composition gas (Wood et al. 2019), showing a tendency for the refractory elements to be enhanced in the Sun compared with the meteorites. Only elements with a combined uncertainty of $<0.1$ dex in the abundance difference are shown in the figure. It should be noted that $\mathrm{C}, \mathrm{N}$, and $\mathrm{O}$ (condensation temperature $<200 \mathrm{~K}$ ) fall outside the plot with the meteoritic abundances depleted compared to the Sun, as is also the case for the noble gases. The solid line is a weighted least-squares fit to the data with a slope of $(6.9 \pm 2.7) \cdot 10^{-5}$ dex $\mathrm{K}^{-1}$.

intercomparing relative abundances of similar objects analysed homogeneously, such as solar/stellar twins or chondrites. A weighted linear least-squares fit implies $\Delta \log \epsilon=(6.9 \pm 2.7)$. $10^{-5} T_{\text {cond }}+(-0.09 \pm 0.04)$, and hence the correlation does not quite reach $3 \sigma$ significance; with the condensation temperatures of Lodders (2003), the slope would be $(7.3 \pm 2.6) \cdot 10^{-5}$ dex $\mathrm{K}^{-1}$. Including all elements except $\mathrm{Li}$ and the highly volatile elements hardly changes the best fit. The quality of the current abundance data does not motivate a more sophisticated function, such as piece-wise linear and step functions, as attempted in related cases involving dust condensation (e.g., Meléndez et al. 2009; Gonzalez 2014).

Compared to CI chondrites, other types of carbonaceous chondrites show increasing fractionation with condensation temperature $(\mathrm{CI} \rightarrow \mathrm{CM} \rightarrow \mathrm{CR} \rightarrow \mathrm{CO} \rightarrow \mathrm{CV} \rightarrow \mathrm{CB} \rightarrow \mathrm{CH}$, Wasson \& Kallemeyn 1988; Krot et al. 2014). Since Palme et al. (2014) did not provide data for other classes of chondrites, we made use of the published abundances by Bland et al. (2005) for 42 elements, re-normalised and converted to the astronomical logarithmic abundance scale. An advantage with the Bland et al. (2005) dataset is that they tabulate the compositions both for the bulk of the chondritic material as well as the fine-grained matrix of the meteorites, which are important for our discussion below. The abundances for other chondrite classes are given normalised to $\mathrm{CI}$ (and the element $\mathrm{Yb}$ ). While the relative Si abundances are provided for the matrix components, they are missing for the bulk material for non-CI chondrites for reasons unclear to us. As a result, when comparing the bulk compositions we have had to normalise to the astronomical abundance scale using $\mathrm{Mg}$ as the reference element, which introduces a 0.02 dex shift; as will become clear, this minor offset is unimportant for our conclusions.

The resulting CI abundances from Bland et al. (2005) are not identical to those adopted in Sect. 3.1, but the differences are in most cases minor, as seen in Fig. 7. Importantly, the correla-
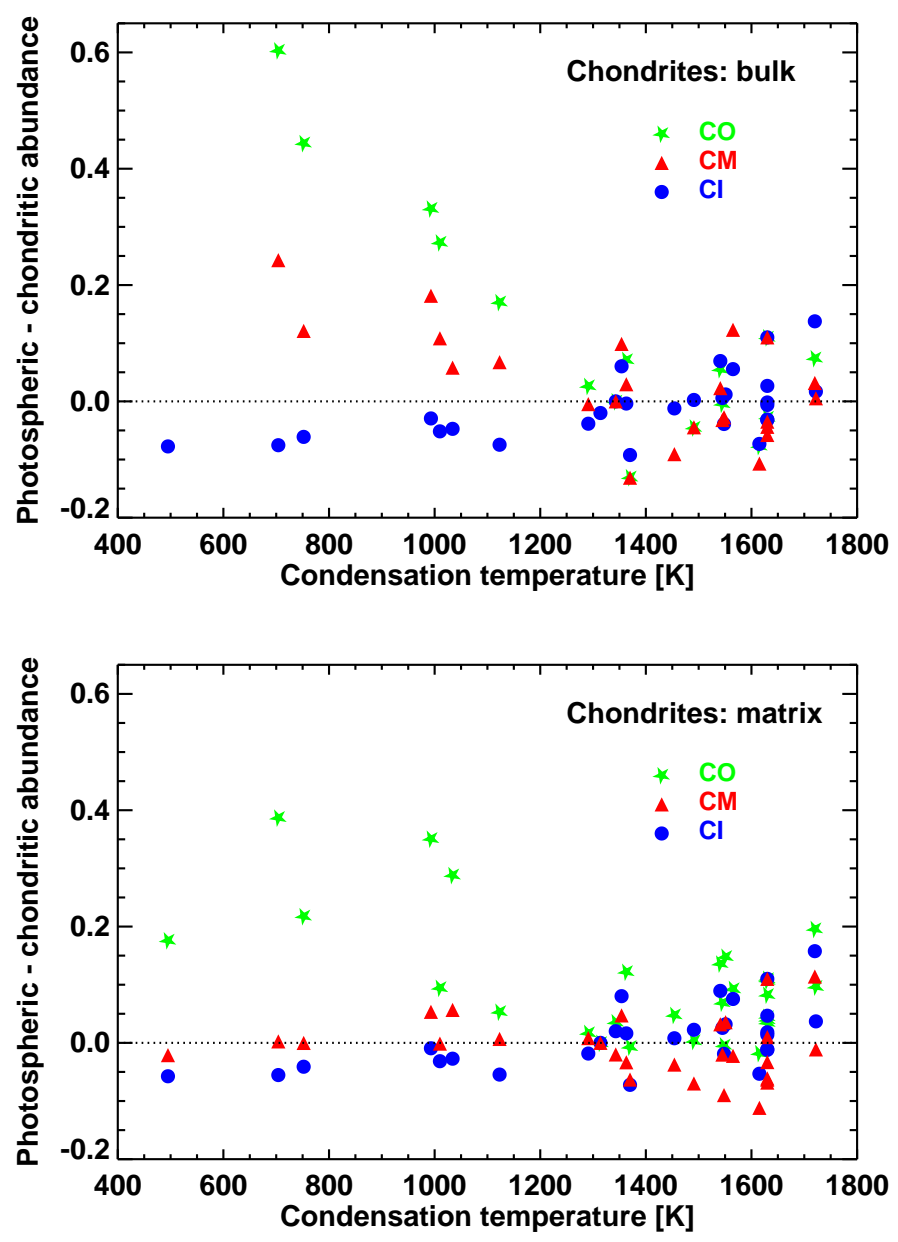

Fig. 7. Upper panel: difference between the solar photospheric and bulk abundances for $\mathrm{CI}$ (blue circles), CM (red triangles), and CO (green stars) chondrites as measured by Bland et al. (2005). CM and CO chondrites show increasing fractionation compared to the Sun, especially for the volatile elements. Lower panel: same, but for the fine-grained matrix of chondrites. Surprisingly, the solar abundances more closely resemble the CM matrix than the CI equivalent, which shows a trend with condensation temperature. As explained in the text, for the bulk comparison $\mathrm{Mg}$ has been used as the reference element to connect the meteoritic and photospheric abundance scales since the $\mathrm{Si}$ abundances are not provided by Bland et al. (2005) for non-CI chondrite classes, which introduces a 0.02 dex offset. For the matrix compositions, the usual reference element $\mathrm{Si}$ has been used.

tion with condensation temperature is still apparent even if data for some elements, including the moderately volatile $\mathrm{Na}$ and $\mathrm{S}$, are lacking; the weighted difference between the Sun and the Bland et al. (2005) CI data for the moderately volatile elements is $-0.040 \pm 0.007$ (s.d. $=0.018$ ) dex, which is almost identical to the case with the Palme et al. (2014) CI abundances adopted elsewhere in our study. In terms of their bulk abundances, the CI chondrites are clearly the most solar-like, the trend with condensation temperature notwithstanding, followed by the $\mathrm{CM}$ and $\mathrm{CO}$ meteorites, which show increasing depletion of the volatile elements, while the refractory elements display less of a variation relative to the Sun and CI (Fig. 7); the highly volatile elements $(\mathrm{H}, \mathrm{C}, \mathrm{N}, \mathrm{O}$, and noble gases) not included in the figure are even more depleted in the non-CI chondrites.

An important distinction between the chondrite classes is that CI meteorites are only comprised of the fine-grained matrix, while other chondrites also consist of chondrules and refractory 
inclusions with a volatile-depleted/refractory-enriched composition relative to their matrices; the fraction of matrix material varies between the classes as well as between meteorites of the same type. Chondrules have experienced rapid heating to temperatures $>1400 \mathrm{~K}$, followed by similarly quick cooling, likely in the inner part of the proto-planetary disc, but the details of their formation and subsequent incorporation into the matrix is still hotly debated; the matrix material has not experienced any significant heating events. The chemical complementarity between the chondrules and matrix that has been previously argued for (e.g., Bland et al. 2005; Palme \& Hezel 2011) has more recently been questioned, implying that the chondrules and matrix did not necessarily form from the same reservoir (van Kooten et al. 2019; Alexander 2019). To facilitate a fair comparison with the solar abundances, one should therefore consider the chondritic matrices, which are clearly more pristine than the chondrules, rather than their bulk compositions.

The lower panel of Fig. 7 compares the matrix compositions of $\mathrm{CI}, \mathrm{CM}$, and $\mathrm{CO}$ chondrites relative to the Sun using data from Bland et al. (2005). Since the chondritic matrix contains a higher abundance of volatile elements than chondrules, the matrices are more similar to the Sun. Remarkably, the CM matrix is overall a very good match to the present-day solar photosphere, closer in fact than the CI chondrites. In particular, the moderately volatile elements agree better with a weighted mean difference of $+0.014 \pm 0.010$ (s.d. $=0.027$ ) dex, while the refractory elements show similar differences for CI and CM matrix although of opposite sign; in both cases, the main component elements agree excellently with the Sun. The weighted linear least-squares fit for the CM matrix data has a slope of $(-4.6 \pm 3.6) \cdot 10^{-5} \mathrm{dex} \mathrm{K}^{-1}$, while the corresponding slope for CI chondrites is $(8.6 \pm 3.6) \cdot 10^{-5}$ dex $\mathrm{K}^{-1}$ with the Bland et al. (2005) elemental abundances. We conclude that CI chondrites are not a perfect compositional match with the Sun, as has been assumed for more than half a century. Instead, the solar abundances more closely resemble those of the matrices of CM chondrites, although not the bulk CM composition. This could reflect spatially separate origins of the $\mathrm{CM}$ matrix and chondrules with differing chemical make-up, with some chemical complementarity possibly emerging later following transport to where amalgamation occur (van Kooten et al. 2019). A more detailed comparison will be required to elucidate how, where and when the different chondrite constituents formed and their relation to the solar composition in this new scenario (e.g., Scott 2007; Alexander 2019; van Kooten et al. 2019). In the meantime, we advise against using meteoritic abundances to estimate the Solar System composition, which is often done due to their generally higher precision (e.g., Vinyoles et al. 2017; Lodders 2019; Wang et al. 2019).

The correlations with chondrites shown in Fig. 7 are reminiscent of the peculiar solar abundances relative to solar twins; the Sun is unusually volatile rich and refractory poor with an abundance amplitude of about 0.08 dex (Meléndez et al. 2009; Ramírez et al. 2010). In other words, the abundance differences relative to the average of solar twins is almost exactly a mirror image of the differences with the CI chondrites. Only $\approx 10 \%$ of the studied stars share the detailed chemical fingerprint with the Sun, which furthermore cannot be explained by differences in age or Galactic birthplace of the stars (Bedell et al. 2018; Nissen \& Gustafsson 2018). The correlation with condensation temperature suggests that the signature is related to planet formation. The initial suggestion that the abundance pattern is imprinted by the formation of terrestrial planets in the Solar System (Meléndez et al. 2009; Chambers 2010) is likely not cor- rect since the solar convection zone was much deeper during the proto-planetary disc phase compared to today, thus requiring much more than a few $\mathbf{M}_{\oplus}$ of refractory-rich material locked up in rocky planets. Furthermore, transit data from Kepler imply that most solar-like stars harbour super-Earths or mini-Neptunes more massive than the terrestrial planets in the Solar System (Zink et al. 2019), which would make the Sun stand out in the opposite manner compositionally. The alternative explanation that other stars have on average experienced more accretion of rocky planets is also unlikely to hold up due to the rapid erasure of such metal-rich accretion from the stellar surface convection zone due to thermohaline mixing (Théado \& Vauclair 2012).

Recently, a more promising scenario was proposed by Booth \& Owen (2020), which involves the early formation of giant planets trapping $>100 M_{\oplus}$ of refractory-rich dust external to their orbits while volatile-rich gas continues to accrete onto the proto-star. They found that such gas-dust separation could imprint an abundance pattern similar to that observed when the Sun is deficient in refractory elements by $\approx 0.08 \mathrm{dex}$ (20\%). The prediction is thus that most solar twins lack a gas giant planet, allowing the gas and dust accretion to continue largely unimpeded. The incidence of the peculiar solar abundance pattern is consistent with the frequency of giant planets being $\approx 7 \%$ (Wittenmyer et al. 2020). Long-term precision radial velocity monitoring of both stars most similar and most chemically disparate to the Sun should be undertaken to search for Jupiter-like planets; HIP11915 is also a chemically solar twin that hosts a Jupiter twin (Yana Galarza et al. 2021), although the planet hosts HIP5301 and HIP15227 seemingly do not fit the pattern (Bedell et al. 2018) - a much larger sample is obviously needed. The connection with the chondritic compositions discussed above also needs to be deciphered within the framework of an early formation of Jupiter.

\section{Isotopic abundances}

Isotopic abundances can only be measured from solar spectroscopy for $\mathrm{C}$ and $\mathrm{O}$, and even then only with significant uncertainties (Scott et al. 2006; Ayres et al. 2013; Lyons et al. $2018)^{13}$. Instead, alternative methods are used to infer the solar values, including sample return missions of the solar wind such as NASA's Genesis probe (Burnett et al. 2003; Jurewicz et al. 2003) and laboratory measurements of various terrestrial, lunar, meteoritic, cometary, and asteroid samples. Our advocated isotopic abundances can be found in Table B.1.

The present-day solar $\mathrm{D}$ abundance can not be directly determined due to near-complete burning of $\mathrm{D}$ to ${ }^{3} \mathrm{He}$ during the pre-main-sequence evolution. The increase from the proto-solar ${ }^{3} \mathrm{He} /{ }^{4} \mathrm{He}$ ratio as indicated by the value measured in Jupiter $\left(1.66 \pm 0.05 \cdot 10^{-4}\right.$, Mahaffy et al. 1998) and the local interstellar cloud $\left(1.62 \pm 0.29 \cdot 10^{-4}\right.$, Busemann et al. 2006), to the present-day ratio in the solar wind is thus a reflection of the proto-solar $\mathrm{D} / \mathrm{H}$ ratio. The isotope fluences determined from the Genesis probe suggest ${ }^{3} \mathrm{He} /{ }^{4} \mathrm{He}=(4.5-4.8) \cdot 10^{-4}$ depending on the solar wind regime (Heber et al. 2012), which indicates the presence of isotopic fractionation during the formation and acceleration of the solar wind. Two methods to account for fractionation are presented in Heber et al. (2012): theoretical corrections based on the inefficient Coulomb drag model or those based on empirical correlations between ${ }^{3} \mathrm{He} /{ }^{4} \mathrm{He}$ and

13 The Li I $670.8 \mathrm{~nm}$ line in the Sun is unfortunately too weak to facilitate a determination of the photospheric ${ }^{6} \mathrm{Li} /{ }^{7} \mathrm{Li}$ isotopic ratio, as has been possible in some stars (e.g., Lind et al. 2013; Mott et al. 2017). 


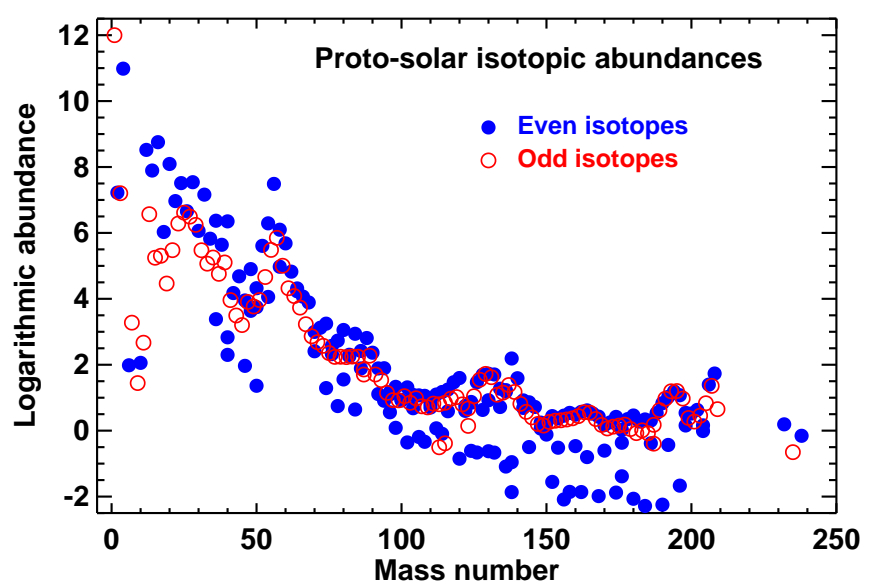

Fig. 8. Proto-solar isotopic abundances as a function of mass number after taking into account atomic diffusion for $\mathrm{He}(+0.07 \mathrm{dex})$ and heavier elements $(+0.06 \mathrm{dex})$ and radioactive decay. Even isotopes are shown as filled blue circles and odd isotopes as open red circles.

$\mathrm{H} /{ }^{4} \mathrm{He}$ in the solar wind, which is similar to our determination of the solar Ne and Ar absolute abundances. Substantially different present-day ${ }^{3} \mathrm{He} /{ }^{4} \mathrm{He}$ ratios and thus proto-solar $\mathrm{D} / \mathrm{H}$ abundances are obtained: $1.24 \cdot 10^{-5}$ (theoretical) and $1.67 \cdot 10^{-5}$ (empirical). We take the average of the two values together with the Jovian $\mathrm{D} / \mathrm{H}\left(2.1 \cdot 10^{-5}\right.$, Lellouch et al. 2001) to obtain a proto-solar $\mathrm{D} / \mathrm{H}$ ratio of $(1.67 \pm 0.25) \cdot 10^{-5}$. We caution that the here-derived proto-solar $\mathrm{D} / \mathrm{H}$ value is in contradiction with the present-day $\mathrm{D}$ abundance in the local interstellar medium of $\mathrm{D} / \mathrm{H} \geq(2.0 \pm 0.1) \cdot 10^{-5}$ (Linsky et al. 2006; Prodanović et al. 2010) since astration of D in stars should result in a monotonic decrease with time. The here-deduced proto-solar D would also lead to uncomfortably large astration factors from the primordial value of $(2.53 \pm 0.03) \cdot 10^{-5}$ as inferred from high-redshift, metalpoor damped Ly-alpha systems (Weinberg 2017; Cooke et al. 2018). The proto-solar $\mathrm{D} / \mathrm{H}$ isotopic ratio clearly deserves further scrutiny.

The ${ }^{12} \mathrm{C} /{ }^{13} \mathrm{C}$ ratio does not appear to vary significantly between different Solar System objects. We therefore adopt the reference terrestrial value of $89.3 \pm 0.2$ (Meija et al. 2016b), which is consistent with the solar photospheric determination (Scott et al. 2006; Lyons et al. 2018) and the preliminary Genesis results for the solar wind (Wiens et al. 2013).

The ${ }^{14} \mathrm{~N} /{ }^{15} \mathrm{~N}$ ratio varies tremendously between Solar System sources for reasons that are still poorly understood. The Genesis team has measured the isotopic ratio in the solar wind (Marty et al. 2010), which with an isotopic fractionation correction (Laming et al. 2017) implies ${ }^{14} \mathrm{~N} /{ }^{15} \mathrm{~N}=453 \pm 66$. A similar value, $435 \pm 57$, is obtained for Jupiter (Owen et al. 2001). We take the weighted mean of these two measurements: ${ }^{14} \mathrm{~N} /{ }^{15} \mathrm{~N}=$ $443 \pm 13$.

The isotopic composition of $\mathrm{O}$ in the solar wind was measured by McKeegan et al. (2011) using the Genesis sample return mission. After correction for isotopic fractionation in the solar wind, they estimated solar isotopic ratios of ${ }^{16} \mathrm{O} /{ }^{18} \mathrm{O}=530$ and ${ }^{16} \mathrm{O} /{ }^{17} \mathrm{O}=2798$, which we adopt here. The most recent spectroscopic analyses of CO lines in the Sun with 3D atmosphere models are consistent with these ratios, albeit with relatively large uncertainties (Lyons et al. 2018). Like almost all rocky material in the inner Solar System, the Earth is enriched in ${ }^{18} \mathrm{O}$ and ${ }^{17} \mathrm{O}$ in comparison to ${ }^{16} \mathrm{O}$ (isotopic ratios of 499 and 2632, respectively, Meija et al. 2016b), likely as a result of CO self-shielding during the proto-planetary disc phase of the Solar System's formation (Clayton 2002; McKeegan et al. 2011).

Genesis solar wind data also allow the isotopic abundances of the other noble gases $\mathrm{Ne}, \mathrm{Ar}, \mathrm{Kr}$, and $\mathrm{Xe}$ to be determined. For $\mathrm{Ne}$ and $\mathrm{Ar}$, the isotopic fractionations are significantly smaller than for $\mathrm{He}$, while for $\mathrm{Kr}$ and $\mathrm{Xe}$ they can be neglected altogether (Heber et al. 2012). Using the empirical correlations to correct for the fractionation, the present-day solar isotopic ratios are ${ }^{20} \mathrm{Ne} /{ }^{22} \mathrm{Ne}=13.36 \pm 0.09,{ }^{21} \mathrm{Ne} /{ }^{22} \mathrm{Ne}=0.0324$, and ${ }^{36} \mathrm{Ar} /{ }^{38} \mathrm{Ar}=$ $5.37 \pm 0.03$ (Heber et al. 2012; Meshik et al. 2014); almost identical values are obtained with the inefficient Coulomb drag theoretical model (Bodmer \& Bochsler 2000). To our knowledge, the ${ }^{36} \mathrm{Ar} /{ }^{40} \mathrm{Ar}$ ratio has not been determined from the Genesis samples and hence the proto-solar ${ }^{40} \mathrm{Ar}$ abundance is highly uncertain (Anders \& Grevesse 1989); the terrestrial abundance is completely dominated by the radioactive decay of ${ }^{40} \mathrm{~K}$ (Meija et al. 2016b), making it the most abundant isotope. For $\mathrm{Kr}$ and $\mathrm{Xe}$, we embrace the Genesis results from Meshik et al. (2014) and Meshik et al. (2020), respectively.

For the non-volatile elements, the Genesis solar wind samples have not yet delivered final isotopic abundances, although this should be feasible for elements like $\mathrm{Mg}, \mathrm{Si}, \mathrm{S}$, and $\mathrm{Fe}$ in due course (Burnett et al. 2019). Mg is particularly interesting since it enables a critical test of the solar wind isotopic fractionation models (e.g., Bodmer \& Bochsler 2000; Laming et al. 2017), which are in reasonable agreement with the most recent Genesis results (Jurewicz et al. 2020). Fe isotopic measurements of the Genesis data on the other hand would test the notion of a chemically homogeneous proto-solar nebula, that is, whether the Sun and, for example, the most chemically pristine meteorites are expected to have identical composition as normally assumed (Sect. 3).

For the elements not discussed above, we take the presentday relative isotopic fractions from the recommendations by the International Union of Pure and Applied Chemistry (Meija et al. 2016 b with subsequent updates for $\mathrm{Yb}$, Ta, Ir, and $\mathrm{Hf}^{14}$ ) for representative terrestrial samples (the mean when a range is given). The proto-solar isotopic fractions given in Table B.1 are assumed to be the same as the present-day values, with the exception of radioactive elements and their daughter products; we adopt the recommended isotopic half-lives from IAEA Nuclear Data Services ${ }^{15}$ and a Solar System age of $4.568 \mathrm{Gyr}$ (Bouvier \& Wadhwa 2010; Connelly et al. 2012).

\section{Present-day and proto-solar Solar System abundances}

With the Sun providing $>99.85 \%$ of the total mass in the Solar System, we can safely adopt the solar abundances as the basis of the chemical composition of the solar system overall. The present-day photospheric elemental abundances, however, are not the same the Sun was born with due to three main effects: nuclear processing, radio-active decay, and diffusion. The first effect only impacts $\mathrm{Li}$ (and possibly Be), which has been largely destroyed in the convection zone, as is evident from the comparison with the most pristine meteorites (Sect. 3.2). No nuclear products from $\mathrm{H}$-burning in the solar core have reached the solar surface layers, with the exception of D-burning in the deep convection zone during the pre-main sequence (Sect. 4). Secondly, elements such as Th and $U$ are radioactively unstable,

\footnotetext{
14 https://WwW.ciaaw.org/isotopic-abundances.htm 15 https://www-nds.iaea.org/relnsd/NdsEnsdf/QueryForm. html
} 

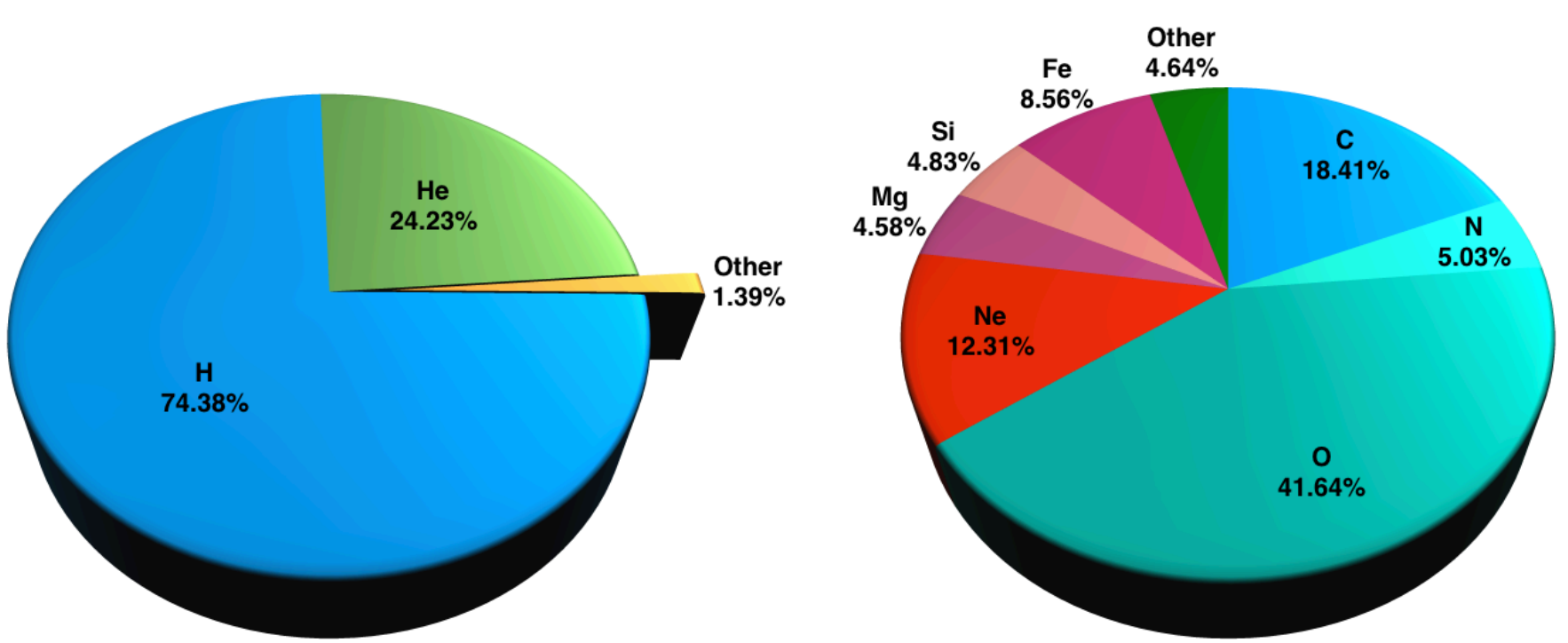

Fig. 9. Present-day photospheric mass fractions the most abundant elements. Left panel: mass fractions of $\mathrm{H}, \mathrm{He}$, and heavy elements. Right panel: relative mass fractions of the most abundant heavy elements with the highly volatile $\mathrm{C}, \mathrm{N}, \mathrm{O}$, and Ne contributing more than $77 \%$ of all heavy elements by mass.

and hence their proto-solar abundances 4.57 Gyr ago were larger than measured today; as noted in Sect. 4, we account for this using the recommended half-lives. Finally, all elements heavier than $\mathrm{H}$ have partly settled from the surface convection zone to the radiative interior from the combined effects of microscopic thermal diffusion, gravitational settling, and radiative acceleration, normally together referred to as atomic diffusion (Thoul et al. 1994).

Quantitative predictions for elemental diffusion are hampered by the possibility of additional mixing below the convection zone due to still poorly understood processes like convective overshooting, rotation, internal gravity waves, and turbulence (e.g., Goldreich \& Schubert 1967; Chaboyer et al. 1995; Baraffe et al. 2017; Aerts et al. 2019; Dumont et al. 2021), which is required to explain the solar $\mathrm{Li}$ abundance and interior rotation profile. Different groups thus require slightly varying proto-solar abundances depending on the ingredients of their stellar evolution codes when calibrating to the solar luminosity, effective temperature and surface composition at the solar age. Here we adopt the predictions from the standard solar model of Vinyoles et al. (2017) computed with the Asplund et al. (2009) chemical composition. In terms of number densities relative to $\mathrm{H}$, the proto-solar $\mathrm{He}$ abundance is higher by $0.070 \mathrm{dex}$ compared with the present-day photospheric value, while for all heavier elements the difference is 0.064 dex. Because the Vinyoles et al. (2017) solar model does not include any additional mixing beyond atomic diffusion as required to explain the solar Li depletion, these diffusion corrections may be slightly over-estimated; more recent solar models which simultaneously agree better with the Li depletion and helioseismology (see below) have diffusion abundance corrections about half of this: $\approx 0.03$ dex for all elements other than $\mathrm{H}$ (Eggenberger et al. 2021).

Regarding elements for which no photospheric abundance determination is feasible from spectroscopy or solar wind measurements (Table 2), we base their present-day photospheric abundances on the CI chondritic abundances. Those are first corrected for the trend in abundance difference with condensation temperature (Fig. 6, Sect. 3.3) before applying the effects of atomic diffusion to compute the proto-solar abundances. The proto-solar isotopic abundances are given in Table B.1.
In terms of mass fractions of $\mathrm{H}, \mathrm{He}$, and heavy elements, the elemental abundances presented in Table 2 correspond to present-day photospheric values of $X_{\text {surface }}=0.7438 \pm 0.0054$, $Y_{\text {surface }}=0.2423 \pm 0.0054, Z_{\text {surface }}=0.0139 \pm 0.0006$, and $Z_{\text {surface }} / X_{\text {surface }}=0.0187 \pm 0.0009$; Fig. 9 illustrates the presentday photospheric mass fractions of the most abundant elements. The quoted uncertainty on $Y_{\text {surface }}$ stems from the helioseismic determination (Sect. 2.8), while for the heavier elements we assumed that their abundance errors to be independent. The here inferred solar surface metallicity is slightly higher than (but within the uncertainties of $)$ the value $\left(Z_{\text {surface }}=0.0134\right)$ derived in Asplund et al. (2009), mainly as a result of the revised $\mathrm{Ne}$ abundance following the adoption of the Genesis solar wind measurements (Sect. 2.8). With the Vinyoles et al. (2017) solar model for atomic diffusion, the proto-solar mass fractions would be $X_{\text {initial }}=0.7121, Y_{\text {initial }}=0.2725$, and $Z_{\text {initial }}=0.0154$, while with a smaller abundance correction of $0.03 \mathrm{dex}$ for all elements heavier than $\mathrm{H}$ (Eggenberger et al. 2021) the values are $X_{\text {initial }}=0.7304, Y_{\text {initial }}=0.2550$, and $Z_{\text {initial }}=0.0146$. Table 4 lists the inferred present-day photospheric and proto-solar mass fractions based on some widely used compilations of the solar chemical composition over the past three decades.

\section{Solar abundances, standard solar models, and helioseismology}

Care must be exercised when interpreting proto-solar mass fractions in light of the long-standing and exasperating discrepancy between helioseismology and predictions from standard solar models constructed with present-day surface abundances similar to those presented here: the so-called solar modelling problem ${ }^{16}$ (e.g., Basu \& Antia 2004; Bahcall et al. 2005; Vinyoles et al. 2017; Christensen-Dalsgaard 2021). In particular, the comparatively low photospheric $\mathrm{C}, \mathrm{N}$, and $\mathrm{O}$ abundances (and associated $\mathrm{Ne}$ abundance) from 3D-based spectroscopic analyses (e.g., Allende Prieto et al. 2001b, 2002; Asplund et al. 2004, 2005b, 2009; Amarsi et al. 2018a, 2019,

16 While sometimes referred to as the solar abundance problem, we argue that this is a misnomer since it is not at all clear that the solar composition is at fault. 
Table 4. Present-day photospheric and proto-solar mass fractions of hydrogen (X), helium (Y), and metals (Z) for a number of widely used compilations of the solar chemical composition.

\begin{tabular}{|c|c|c|c|c|c|c|c|c|}
\hline \multirow[t]{2}{*}{ Source } & \multicolumn{4}{|c|}{ Present-day photospheric } & \multicolumn{4}{|c|}{ Proto-solar } \\
\hline & $X_{\text {surface }}$ & $Y_{\text {surface }}$ & $Z_{\text {surface }}$ & $Z_{\text {surface }} / X_{\text {surface }}$ & $X_{\text {initial }}$ & $Y_{\text {initial }}$ & $Z_{\text {initial }}$ & $Z_{\text {initial }} / X_{\text {initial }}$ \\
\hline Present work & 0.7438 & 0.2423 & 0.0139 & 0.0187 & 0.7121 & 0.2725 & 0.0154 & 0.0216 \\
\hline Lodders 2019 & 0.7389 & 0.2463 & 0.0148 & 0.0200 & 0.7061 & 0.2766 & 0.0173 & 0.0245 \\
\hline Caffau et al. $2011^{(a)}$ & 0.7321 & 0.2526 & 0.0153 & 0.0209 & & & & \\
\hline Asplund et al. 2009 & 0.7381 & 0.2485 & 0.0134 & 0.0181 & 0.7154 & 0.2703 & 0.0142 & 0.0199 \\
\hline Lodders et al. 2009 & 0.7390 & 0.2469 & 0.0141 & 0.0191 & 0.7112 & 0.2735 & 0.0153 & 0.0215 \\
\hline Asplund et al. 2005a & 0.7392 & 0.2485 & 0.0122 & 0.0165 & 0.7166 & 0.2704 & 0.0130 & 0.0181 \\
\hline Lodders 2003 & 0.7491 & 0.2377 & 0.0133 & 0.0177 & 0.7111 & 0.2741 & 0.0149 & 0.0210 \\
\hline Grevesse \& Sauval 1998 & 0.7347 & 0.2483 & 0.0169 & 0.0231 & 0.7062 & 0.2750 & 0.0188 & 0.0266 \\
\hline Grevesse et al. 1993 & 0.7028 & 0.2800 & 0.0172 & 0.0245 & & & & \\
\hline Anders \& Grevesse 1989 & 0.7065 & 0.2741 & 0.0194 & 0.0274 & & & & \\
\hline
\end{tabular}

Notes. ${ }^{(a)}$ Caffau et al. (2011) determined the abundances of Li, C, N, O, P, S, K, Fe, Eu, Hf, Os, and Th and adopted the recommended values of Lodders et al. (2009) for all other elements. They did not quote any proto-solar mass fractions.

2020) have ruined the good agreements with helioseismology that were obtained with global solar models computed with the older higher solar metallicity (e.g., Anders \& Grevesse 1989: $Z_{\text {surface }} / X_{\text {surface }}=0.274 ;$ Grevesse et al. $1993: Z_{\text {surface }} / X_{\text {surface }}=$ 0.246; Grevesse \& Sauval 1998: $\left.Z_{\text {surface }} / X_{\text {surface }}=0.231\right)$. The predicted surface He abundance, depth of the convection zone, and the sound speed as a function of solar radius from a solar calibration are now significantly more discrepant compared to the inversion of the observed solar p-mode oscillation frequencies; the solar surface composition presented here goes in the right direction but will not resolve the mismatch. We note, however, that the available seismic data in fact favours a low metallicity ( $\left.Z_{\text {surface }}<0.014\right)$ in the convection zone (Vorontsov et al. 2013; Buldgen et al. 2017), suggesting that current standard solar models are missing important physics or are constructed with faulty input data in the radiative zone below the convection zone.

A huge body of work has been devoted to reconciling the spectroscopic and interior solar models triggered by our 3Dbased solar abundances over the past two decades. Arguably the most straightforward explanation would be underestimated opacities, especially near the bottom of the convection zone where the discrepancies are most acute, to compensate for the lowering of the abundances of the dominant elements. It would require the computed data sets of atomic opacities (e.g., Badnell et al. 2005; Mondet et al. 2015; Colgan et al. 2016) all to be underestimated by about $20 \%$ in the relevant temperature-pressure regime (Serenelli et al. 2009; Christensen-Dalsgaard et al. 2009). In fact recent experimental opacity measurements are systematically higher than calculations with the missing opacity for $\mathrm{Fe}$ alone accounting for about half of what is required to resolve the solar modelling problem (Bailey et al. 2015; Nagayama et al. 2019). With new experiments and updated calculations with previously overlooked atomic processes underway (e.g., Nahar \& Pradhan 2016; Krief et al. 2018) it remains to be seen whether the final atomic opacities will be sufficient. Important independent constraints on the solar interior composition will also come from measurements of the solar neutrino fluxes, especially those produced in the CNO-cycle that were recently reported by the Borexino experiment (Borexino Collaboration 2020). Finally, encouraging signs are appearing from new solar models with improved mixing below the convection zone aimed at explaining the depletion of $\mathrm{Li}$ and the interior rotation profile, which standard models cannot do (e.g., Yang 2019; Zhang et al. 2019; Buldgen et al. 2019a,b; Eggenberger et al. 2021). We remain cautiously optimistic that the persistent solar modelling problem will soon have been resolved without necessitating any major revision of the photospheric solar abundances presented here.

\section{Concluding remarks}

The elemental abundances derived and presented here are a further stepping stone towards the advancement of our understanding of the solar chemical composition, which is of such crucial importance for astronomy as a whole. As demonstrated above, major improvements in terms of the solar spectroscopic analysis have been accomplished, which should make the inferred elemental abundances both more precise and more accurate. It is now perfectly feasible to perform detailed 3D non-LTE line formation calculations using highly realistic 3D radiativehydrodynamical simulations of the solar surface convection and atmosphere. This has been achieved for 13 elements - Li, C, $\mathrm{N}, \mathrm{O}, \mathrm{Na}, \mathrm{Mg}, \mathrm{Al}, \mathrm{Si}, \mathrm{K}, \mathrm{Ca}, \mathrm{Mn}, \mathrm{Fe}$, and $\mathrm{Ba}$ - all using comprehensive model atoms with reliable atomic data, including the previously notoriously uncertain cross-sections for $\mathrm{H}$ collisions. Compared to Asplund et al. (2009), in which only the abundances of $\mathrm{Li}$ and $\mathrm{O}$ were based on a consistent 3D nonLTE analysis, this certainly represents substantial progress. The abundances of a further 45 elements have been determined on the basis of a 3D LTE spectroscopic analysis. In total, we present the solar photospheric abundances of 68 elements when also including the noble gases, for which helioseismic and solar wind measurements are available, and four trace elements evaluated from sunspot observations. Importantly, the abundance uncertainties for these 68 elements have been carefully evaluated, including both statistical and systematic uncertainties. These have been augmented with meteoritic data (corrected for an identified trend with condensation temperature) for a further 15 elements, thus providing Solar System abundances for all 83 long-lived elements.

Based on our state-of-the-art 3D non-LTE analysis, we can safely confirm that the old high solar metallicity of, for example, Grevesse et al. (1993) and Grevesse \& Sauval (1998), which are still used to construct standard solar models, should finally be discarded - there are simply no arguments in favour of those outdated 1D LTE results spectroscopically. Our study is, however, definitely not the final word on the issue of the solar 
chemical composition, with much work remaining to reach comparable abundance precision to that of the most pristine meteorites. Arguably, the most pressing need is to extend non-LTE calculations to additional elements: only 26 elements have their solar abundances determined from a non-LTE analysis, half of which are only in 1D. Of particularly high priority is to carry out 3D non-LTE computations for the remaining Fe-peak elements (including revisiting the important case of $\mathrm{Mn}$, Sect. 2.5) as well as other elements of key astronomical significance, such as $\mathrm{Be}$ (to constrain solar depletion, Sect. 3.2), S, Zn, Rb (volatile elements to confirm differences with CI chondrites, Sect. 3.3), $\mathrm{P}, \mathrm{Cu}$, and $\mathrm{Sr}$ (stellar nucleosynthesis yields, Kobayashi et al. 2020). Several of these elements already show substantial nonLTE effects in existing 1D non-LTE calculations, which can be expected to be amplified in 3D.

As always, solar spectroscopy is reliant on having access to accurate atomic data. Over the past decade, the situation regarding experimental transition probabilities has improved significantly for the Fe-peak elements in particular, thanks to the dedicated and valiant efforts by a relatively small group of atomic physicists around the world, as discussed in Sect. 2.5. Several other elements have also seen significant updates, however, the need for further work is essentially endless. Here it suffices to mention the case of $\mathrm{Si}$, the element anchoring the meteoritic abundance scale to the solar photospheric values (Sect. 2.4). Besides the need for refined transition probabilities of spectral lines used as diagnostics, there is also a demand for more and better atomic data for lines blending the primary abundance indicators. Since those lines are typically weaker and arise from higher-excitation levels, they are often not even properly identified or, when included in existing atomic calculations, have faulty $g f$-values.

For non-LTE modelling, significantly more atomic data are necessary, including all radiative (bound-bound and bound-free) and collisional (with electrons and hydrogen) transitions coupling the different atomic levels. Great progress has recently been achieved in this regard, especially related to inelastic $\mathrm{H}$ collisions (e.g., Barklem 2016a; Belyaev et al. 2018; Barklem et al. 2021), which has made the non-LTE results much more trustworthy. Crucially, the relevant atomic data now exist for a 3D non-LTE study for almost all of the elements highlighted above; mainly what is lacking is (wo)man-power to take on the necessary but time-consuming 3D non-LTE analyses. What has not yet been fully explored for the Sun is how departures from LTE in one element affect those of other elements or feed back onto the solar atmospheric structure; preliminary calculations suggest that this may well be of importance (Osorio et al. 2020). Furthermore, we caution that magnetic fields have not been included in the 3D solar atmosphere model employed throughout this study. While early work based on imposed vertical magnetic fields implied that the effects could be substantial (Fabbian et al. 2010), more recent 3D LTE calculations based on a 3D MHD solar model with a self-consistent small-scale magnetic dynamo (Rempel 2014) reveal that the impact on solar abundance determination is negligible $(<0.01 \mathrm{dex})$ at least for $\mathrm{C}, \mathrm{N}, \mathrm{O}$, and $\mathrm{Fe}$ (Shchukina \& Trujillo Bueno 2015; Shchukina et al. 2016); this may not hold however for elements in which non-LTE effects are important and thus deserves further scrutiny. Similarly, there is no indication that the inclusion of a chromosphere or other magnetic activity in the solar model would significantly impact the derived abundances for the weak photospheric lines employed here using disc-centre observations of the quiet Sun (Bergemann et al. 2021).

Besides the obvious benefits from having a better definition of a widely used astronomical yardstick, the continuously improved 3D-based solar abundances reported here and in our earlier works (e.g., Asplund et al. 2005a, 2009) have uncovered two surprising peculiarities regarding the solar chemical composition, neither of which have been explained. The inconsistency between the composition at the surface, as measured from spectroscopy, and the interior, as inferred from helioseismology, has now been around for almost two decades in spite of tremendous efforts in reconciling the two with further improvements to the solar modelling. It is crucial to remember that this is not a solar problem but a stellar problem. If indeed the explanation is missing opacity as suggested by recent experiments (Sect. 6), it would imply that current stellar interior and evolution models are not fully trustworthy. Since such stellar models underpin much of the field of astronomy, it would have profound implications. The second solar abundance feature uncovered here, namely that the supposedly most primitive meteorites - the CI chondrites - and the Sun in fact do not share the same composition, implies that a revision of the earliest history of the Solar System is required, given that their equivalence has been taken for granted for more than half a century (Sect. 3.3). The existence of a similar abundance trend of the condensation temperature between the Sun and solar twins suggests that this may well give us a deeper understanding of planet formation around stars in general.

Acknowledgements. We thank all of our wonderful colleagues around the world for highly rewarding collaborations over many years, numerous stimulating discussions about the topics described herein and their implications for astronomy and physics, and for providing necessary input atomic/molecular data, all of which have greatly benefited this work. M. A. gratefully acknowledges generous funding from the Australian Research Council through a Laureate Fellowship (FL110100012) and a Discovery Project (DP150100250). A. M. A. acknowledges support from the Swedish Research Council (VR 2016-03765 and 2020-03940). This research was supported by computational resources provided by the Australian Government through the National Computational Infrastructure (NCI) under the National Computational Merit Allocation Scheme and the ANU Merit Allocation Scheme (project y89). Some of the computations were also enabled by resources provided by the Swedish National Infrastructure for Computing (SNIC) at the Multidisciplinary Center for Advanced Computational Science (UPPMAX) and at the High Performance Computing Center North (HPC2N) partially funded by the Swedish Research Council through grant agreement no. 2018-05973.

\section{References}

Abrams, M. C., Goldman, A., Gunson, M. R., Rinsland, C. P., \& Zander, R. 1996, Appl. Opt., 35, 2747

Aerts, C., Mathis, S., \& Rogers, T. M. 2019, ARA\&A, 57, 35

Alexander, C. M. O. 2019, Geochim. Cosmochim. Acta, 254, 277

Allende Prieto, C., Barklem, P. S., Asplund, M., \& Ruiz Cobo, B. 2001a, ApJ, 558,830

Allende Prieto, C., Lambert, D. L., \& Asplund, M. 2001b, ApJ, 556, L63 Allende Prieto, C., Lambert, D. L., \& Asplund, M. 2002, ApJ, 573, L137

Amarsi, A. M. 2016, PhD Thesis, The Australian National University, Australia Amarsi, A. M., \& Asplund, M. 2017, MNRAS, 464, 264

Amarsi, A. M., Asplund, M., Collet, R., \& Leenaarts, J. 2016a, MNRAS, 455, 3735

Amarsi, A. M., Lind, K., Asplund, M., Barklem, P. S., \& Collet, R. 2016b, MNRAS, 463, 1518

Amarsi, A. M., Barklem, P. S., Asplund, M., Collet, R., \& Zatsarinny, O. 2018a, A\&A, 616, A89

Amarsi, A. M., Nordlander, T., Barklem, P. S., et al. 2018b, A\&A, 615, A139

Amarsi, A. M., Barklem, P. S., Collet, R., Grevesse, N., \& Asplund, M. 2019, A\&A, 624, A111

Amarsi, A. M., Grevesse, N., Grumer, J., et al. 2020, A\&A, 636, A120

Amarsi, A. M., Grevesse, N., Asplund, M., \& Collet, R. 2021, A\&A, in press, https://doi .org/10.1051/0004-6361/202141384

Anders, E., \& Grevesse, N. 1989, Geochim. Cosmochim. Acta, 53, 197

Andersen, T., Poulsen, O., Ramanujam, P. S., \& Petkov, A. P. 1975, Sol. Phys., 44, 257

Anstee, S. D., \& O’Mara, B. J. 1995, MNRAS, 276, 859

Arlandini, C., Käppeler, F., Wisshak, K., et al. 1999, ApJ, 525, 886 
Asplund, M. 2004, A\&A, 417, 769

Asplund, M. 2005, ARA\&A, 43, 481

Asplund, M., Nordlund, A., Trampedach, R., \& Stein, R. F. 1999, A\&A, 346, L17

Asplund, M., Nordlund, A., Trampedach, R., \& Stein, R. F. 2000a, A\&A, 359, 743

Asplund, M., Nordlund, Å., Trampedach, R., Allende Prieto, C., \& Stein, R. F. 2000b, A\&A, 359, 729

Asplund, M., Ludwig, H.-G., Nordlund, Å., \& Stein, R. F. 2000c, A\&A, 359, 669

Asplund, M., Carlsson, M., \& Botnen, A. V. 2003, A\&A, 399, L31

Asplund, M., Grevesse, N., Sauval, A. J., Allende Prieto, C., \& Kiselman, D. 2004, A\&A, 417, 751

Asplund, M., Grevesse, N., Sauval, A. J., Allende Prieto, C., \& Blomme, R. 2005a, A\&A, 431, 693

Asplund, M., Grevesse, N., \& Sauval, A. J. 2005b, in Cosmic Abundances as Records of Stellar Evolution and Nucleosynthesis, eds. T. G. Barnes, III., \& F. N. Bash, ASP Conf. Ser., 336, 25

Asplund, M., Grevesse, N., Sauval, A. J., \& Scott, P. 2009, ARA\&A, 47, 481

Ayres, T. R., Lyons, J. R., Ludwig, H.-G., Caffau, E., \& Wedemeyer-Böhm, S. 2013, ApJ, 765, 46

Badnell, N. R., Bautista, M. A., Butler, K., et al. 2005, MNRAS, 360, 458

Bahcall, J. N., Serenelli, A. M., \& Basu, S. 2005, ApJ, 621, L85

Bailey, J. E., Nagayama, T., Loisel, G. P., et al. 2015, Nature, 517, 56

Balachandran, S. C., \& Bell, R. A. 1998, Nature, 392, 791

Baraffe, I., \& Chabrier, G. 2010, A\&A, 521, A44

Baraffe, I., Pratt, J., Goffrey, T., et al. 2017, ApJ, 845, L6

Barklem, P. S. 2016a, A\&ARv, 24, 9

Barklem, P. S. 2016b, Phys. Rev. A, 93, 042705

Barklem, P. S. 2018, A\&A, 612, A90

Barklem, P. S., \& Collet, R. 2016, A\&A, 588, A96

Barklem, P. S., \& O’Mara, B. J. 1997, MNRAS, 290, 102

Barklem, P. S., O’Mara, B. J., \& Ross, J. E. 1998, MNRAS, 296, 1057

Barklem, P. S., Piskunov, N., \& O'Mara, B. J. 2000, A\&AS, 142, 467

Barklem, P. S., Belyaev, A. K., Dickinson, A. S., \& Gadéa, F. X. 2010, A\&A 519, A20

Barklem, P. S., Belyaev, A. K., Spielfiedel, A., Guitou, M., \& Feautrier, N. 2012 A\&A, 541, A80

Barklem, P. S., Osorio, Y., Fursa, D. V., et al. 2017, A\&A, 606, A11

Barklem, P. S., Amarsi, A. M., Grumer, J., et al. 2021, ApJ, 908, 245

Barrat, J. A., Zanda, B., Moynier, F., et al. 2012, Geochim. Cosmochim. Acta, 83,79

Basu, S. 2016, Liv. Rev. Sol. Phys., 13, 2

Basu, S., \& Antia, H. M. 2004, ApJ, 606, L85

Basu, S., \& Antia, H. M. 2008, Phys. Rep, 457, 217

Baumann, P., Ramírez, I., Meléndez, J., Asplund, M., \& Lind, K. 2010, A\&A, 519, A87

Bautista, M. A., Fivet, V., Ballance, C., et al. 2015, ApJ, 808, 174

Becker, U., Kerkhoff, H., Kwiatkowski, M., et al. 1980, Phys. Lett. A, 76, 125

Bedell, M., Bean, J. L., Meléndez, J., et al. 2018, ApJ, 865, 68

Beeck, B., Cameron, R. H., Reiners, A., \& Schüssler, M. 2013, A\&A, 558, A48

Bell, R. A., Balachandran, S. C., \& Bautista, M. 2001, ApJ, 546, L65

Belmonte, M. T., Pickering, J. C., Ruffoni, M. P., et al. 2017, ApJ, 848, 125

Belyaev, A. K. 2013, Phys. Rev. A, 88, 052704

Belyaev, A. K., \& Voronov, Y. V. 2017, A\&A, 606, A106

Belyaev, A. K., \& Yakovleva, S. A. 2017, A\&A, 606, A147

Belyaev, A. K., Barklem, P. S., Dickinson, A. S., \& Gadéa, F. X. 2010, Phys Rev. A, 81, 032706

Belyaev, A. K., Voronov, Y. V., \& Gadéa, F. X. 2018, ApJ, 867, 87

Bergemann, M., Lind, K., Collet, R., Magic, Z., \& Asplund, M. 2012, MNRAS 427, 27

Bergemann, M., Collet, R., Amarsi, A. M., et al. 2017, ApJ, 847, 15

Bergemann, M., Gallagher, A. J., Eitner, P., et al. 2019, A\&A, 631, A80

Bergemann, M., Hoppe, R., Semenova, E., et al. 2021, MNRAS, in press https://doi.org/10.1093/mnras/stab2160

Biemont, E., Grevesse, N., Hannaford, P., \& Lowe, R. M. 1981, ApJ, 248, 867

Biemont, E., Baudoux, M., Kurucz, R. L., Ansbacher, W., \& Pinnington, E. H. 1991, A\&A, 249, 539

Blackwell, D. E., Lynas-Gray, A. E., \& Smith, G. 1995, A\&A, 296, 217

Blackwell-Whitehead, R., \& Bergemann, M. 2007, A\&A, 472, L43

Bland, P. A., Alard, O., Benedix, G. K., et al. 2005, Proc. Natl. Acad. Sci., 102, 13755

Bochsler, P. 2007, A\&A, 471, 315

Bodmer, R., \& Bochsler, P. 2000, J. Geophys. Res., 105, 47

Böhm-Vitense, E. 1958, Z. Astrophys., 46, 108

Booth, R. A., \& Owen, J. E. 2020, MNRAS, 493, 5079

Bord, D. J., \& Cowley, C. R. 2002, Sol. Phys., 211, 3

Borexino Collaboration (Agostini, M., et al.) 2020, Nature, 587, 577
Bouvier, J. 2008, A\&A, 489, L53

Bouvier, A., \& Wadhwa, M. 2010, Nat. Geosci., 3, 637

Braukmüller, N., Wombacher, F., Hezel, D. C., Escoube, R., \& Münker, C. 2018 , Geochim. Cosmochim. Acta, 239, 17

Bray, I., \& Stelbovics, A. T. 1992, Phys. Rev. A., 46, 6995

Bray, I., \& Stelbovics, A. T. 1995, Comput. Phys. Commun., 85, 1

Brooke, J. S. A., Bernath, P. F., Schmidt, T. W., \& Bacskay, G. B. 2013, J. Quant. Spectr. Rad. Transf., 124, 11

Brooke, J. S. A., Bernath, P. F., Western, C. M., van Hemert, M. C., \& Groenenboom, G. C. 2014a, J. Chem. Phys., 141, 054310a

Brooke, J. S. A., Ram, R. S., Western, C. M., et al. 2014b, ApJS, 210, 23

Brooke, J. S. A., Bernath, P. F., \& Western, C. M. 2015, J. Chem. Phys., 143, 026101a

Buldgen, G., Salmon, S. J. A. J., Noels, A., et al. 2017, MNRAS, 472, 751

Buldgen, G., Salmon, S. J. A. J., Noels, A., et al. 2019a, A\&A, 621, A33

Buldgen, G., Salmon, S., \& Noels, A. 2019b, Front. Astron. Space Sci., 6, 42

Burbidge, E. M., Burbidge, G. R., Fowler, W. A., \& Hoyle, F. 1957, Rev. Mod. Phys., 29, 547

Burnett, D. S., Barraclough, B. L., Bennett, R., et al. 2003, Space Sci. Rev., 105, 509

Burnett, D. S., Jurewicz, A. J. G., \& Woolum, D. S. 2019, Meteorit. Planet. Sci., 54, 1092

Busemann, H., Bühler, F., Grimberg, A., et al. 2006, ApJ, 639, 246

Caffau, E., Ludwig, H.-G., Steffen, M., et al. 2008, A\&A, 488, 1031

Caffau, E., Maiorca, E., Bonifacio, P., et al. 2009, A\&A, 498, 877

Caffau, E., Ludwig, H.-G., Bonifacio, P., et al. 2010, A\&A, 514, A92

Caffau, E., Ludwig, H.-G., Steffen, M., Freytag, B., \& Bonifacio, P. 2011, Sol. Phys., 268, 255

Caffau, E., Ludwig, H.-G., Malherbe, J.-M., et al. 2013, A\&A, 554, A126

Caffau, E., Ludwig, H.-G., Steffen, M., et al. 2015, A\&A, 579, A88

Cameron, A. G. W. 1957, Atomic Energy of Canada Ltd., Pub.

Cameron, A. G. W. 1968, in Origin and Distribution of the Elements, 125

Carlos, M., Meléndez, J., Spina, L., et al. 2019, MNRAS, 485, 4052

Carlsson, M., Rutten, R. J., Bruls, J. H. M. J., \& Shchukina, N. G. 1994, A\&A, 288,860

Cayrel, R., Steffen, M., Chand, H., et al. 2007, A\&A, 473, L37

Chaboyer, B., Demarque, P., \& Pinsonneault, M. H. 1995, ApJ, 441, 865

Chambers, J. E. 2010, ApJ, 724, 92

Charbonnel, C., \& Talon, S. 2005, Science, 309, 2189

Chmielewski, Y., Brault, J. W., \& Mueller, E. A. 1975, A\&A, 42, 37

Christensen-Dalsgaard, J. 2021, Liv. Rev. Sol. Phys., 18, 2

Christensen-Dalsgaard, J., di Mauro, M. P., Houdek, G., \& Pijpers, F. 2009, A\&A, 494, 205

Claas, W. J. 1951, The Composition of the Solar Atmosphere

Clayton, R. N. 2002, Nature, 415, 860

Colgan, J., Kilcrease, D. P., Magee, N. H., et al. 2016, ApJ, 817, 116

Collet, R., Asplund, M., \& Trampedach, R. 2007, A\&A, 469, 687

Collet, R., Nordlund, Ã., Asplund, M., Hayek, W., \& Trampedach, R. 2018, MNRAS, 475, 3369

Connelly, J. N., Bizzarro, M., Krot, A. N., et al. 2012, Science, 338, 651

Cooke, R. J., Pettini, M., \& Steidel, C. C. 2018, ApJ, 855, 102

Cubas Armas, M., Asensio Ramos, A., \& Socas-Navarro, H. 2017, A\&A, 600, A45

Cubas Armas, M., Asensio Ramos, A., \& Socas-Navarro, H. 2020, A\&A, 643, A142

Cunha, K., \& Smith, V. V. 1999, ApJ, 512, 1006

Delbouille, L., Roland, G., \& Neven, L. 1973, Atlas photometrique du spectre solaire de [lambda] 3000 a [lambda] 10000 (Liege: Universite de Liege, Institut d'Astrophysique)

Delbouille, L., Roland, G., Brault, J., \& Testerman, L. 1981, Photometric Atlas of the Solar Spectrum from 1850 to $10000 \mathrm{~cm}-1$ (Kitt Peak National Observatory)

Den Hartog, E. A., Lawler, J. E., Sobeck, J. S., Sneden, C., \& Cowan, J. J. 2011, ApJS, 194, 35

Den Hartog, E. A., Ruffoni, M. P., Lawler, J. E., et al. 2014, ApJS, 215, 23

Den Hartog, E. A., Lawler, J. E., Sneden, C., Cowan, J. J., \& Brukhovesky, A. 2019, ApJS, 243, 33

Doerr, H.-P., Vitas, N., \& Fabbian, D. 2016, A\&A, 590, A118

Drawin, H.-W. 1968, Z. Phys., 211, 404

Dumont, T., Palacios, A., Charbonnel, C., et al. 2021, A\&A, 646, A48

Eggenberger, P., Buldgen, G., Salmon, S., et al. 2021, Nat. Astron., submitted

Fabbian, D., Khomenko, E., Moreno-Insertis, F., \& Nordlund, A. 2010, ApJ, 724, 1536

Farmer, C. B., \& Norton, R. H. 1989, A high-resolution atlas of the infrared spectrum of the sun and the earth atmosphere from space. A compilation of ATMOS spectra of the region from 650 to $4800 \mathrm{~cm}^{-1}(2.3$ to $16 \mu \mathrm{m})$. Vol. I. The sun

Fontenla, J. M., Avrett, E., Thuillier, G., \& Harder, J. 2006, ApJ, 639, 441 
Freytag, B., Ludwig, H. G., \& Steffen, M. 1996, A\&A, 313, 497

Froese Fischer, C. 2006, J. Phys. B, 39, 2159

Gallagher, A. J., Bergemann, M., Collet, R., et al. 2020, A\&A, 634, A55

Garz, T. 1973, A\&A, 26, 471

Garz, T., Holweger, H., Kock, M., \& Richter, J. 1969, A\&A, 2, 446

Goldberg, L., Muller, E. A., \& Aller, L. H. 1960, ApJS, 5, 1

Goldreich, P., \& Schubert, G. 1967, ApJ, 150, 571

Goldschmidt, V. M. 1938, Skrifter Norske Videnskaps - Akademi Oslo. I. Mat. Naturv. Klasse, 4, 1

Goldschmidt, V. M. 1922, Naturwissenschaften, 10, 918

Gonzalez, G. 1997, MNRAS, 285, 403

Gonzalez, G. 2014, MNRAS, 443, L99

Grevesse, N., \& Noels, A. 1993, eds. N. Prantzos, E. Vangioni-Flam, \& M. Casse Origin and Evolution of the Elements, 15-25

Grevesse, N., \& Sauval, A. J. 1973, A\&A, 27, 29

Grevesse, N., \& Sauval, A. J. 1998, Space Sci. Rev., 85, 161

Grevesse, N., \& Sauval, A. J. 1999, A\&A, 347, 348

Grevesse, N., Lambert, D. L., Sauval, A. J., et al. 1991, A\&A, 242, 488

Grevesse, N., Scott, P., Asplund, M., \& Sauval, A. J. 2015, A\&A, 573, A27

Grumer, J., \& Barklem, P. S. 2020, A\&A, 637, A28

Gustafsson, B., Bell, R. A., Eriksson, K., \& Nordlund, A. 1975, A\&A, 42, 407

Gustafsson, B., Edvardsson, B., Eriksson, K., et al. 2008, A\&A, 486, 951

Hall, D. N. B., \& Noyes, R. W. 1969, Astrophys. Lett., 4, 143

Hall, D. N. B., \& Noyes, R. W. 1972, ApJ, 175, L95

Hannaford, P., Lowe, R. M., Grevesse, N., \& Noels, A. 1992, A\&A, 259, 301

Harutyunyan, G., Steffen, M., Mott, A., et al. 2018, A\&A, 618, A16

Hase, F., Wallace, L., McLeod, S. D., Harrison, J. J., \& Bernath, P. F. 2010, J. Quant. Spectr. Rad. Transf., 111, 521

Hayek, W., Asplund, M., Carlsson, M., et al. 2010, A\&A, 517, A49

Heber, V. S., Wieler, R., Baur, H., et al. 2009, Geochim. Cosmochim. Acta, 73, 7414

Heber, V. S., Baur, H., Bochsler, P., et al. 2012, ApJ, 759, 121

Heiter, U., Lind, K., Bergemann, M., et al. 2021, A\&A, 645, A106

Holmes, C. E., Pickering, J. C., Ruffoni, M. P., et al. 2016, ApJS, 224, 35

Holweger, H. 2001, in Joint SOHO/ACE workshop "Solar and Galactic

Composition", ed. R. F. Wimmer-Schweingruber, AIP Conf. Ser., 598, 23

Holweger, H., \& Müller, E. A. 1974, Sol. Phys., 39, 19

Holweger, H., Gehlsen, M., \& Ruland, F. 1978, A\&A, 70, 537

Holweger, H., Bard, A., Kock, M., \& Kock, A. 1991, A\&A, 249, 545

Hubeny, I., Hummer, D. G., \& Lanz, T. 1994, A\&A, 282, 151

Hummer, D. G., Berrington, K. A., Eissner, W., et al. 1993, A\&A, 279, 298

Huss, G. R., Koeman-Shields, E., Jurewicz, A. J. G., et al. 2020, Meteorit. Planet. Sci., 55,326

Ibgui, L., Hubeny, I., Lanz, T., \& Stehlé, C. 2013, A\&A, 549, A126

Israelian, G., Delgado Mena, E., Santos, N. C., et al. 2009, Nature, 462, 189

Johansson, S., Litzén, U., Lundberg, H., \& Zhang, Z. 2003, ApJ, 584, L107

Jurewicz, A. J. G., Burnett, D. S., Wiens, R. C., et al. 2003, Space Sci. Rev., 105 , 535

Jurewicz, A. J. G., Rieck, K. D., Hervig, R., et al. 2020, Meteorit. Planet. Sci., 55,352

King, A. J., Phillips, K. J. H., Strekopytov, S., Vita-Finzi, C., \& Russell, S. S. 2020, Geochim. Cosmochim. Acta, 268, 73

Kiselman, D. 1991, A\&A, 245, L9

Kiselman, D. 1994, A\&A, 286, 169

Kiselman, D., \& Nordlund, A. 1995, A\&A, 302, 578

Kobayashi, C., Karakas, A. I., \& Lugaro, M. 2020, ApJ, 900, 179

Kock, M., \& Richter, J. 1968, ZAp, 69, 180

Korn, A. J., Shi, J., \& Gehren, T. 2003, A\&A, 407, 691

Korotin, S. A. 2020, Astron. Lett., 46, 541

Kramida, A. 2019, in APS Meeting Abstracts, 2019, N09.004

Krief, M., Kurzweil, Y., Feigel, A., \& Gazit, D. 2018, ApJ, 856, 135

Krot, A. N., Keil, K., Scott, E. R. D., Goodrich, C. A., \& Weisberg, M. K. 2014 in Classification of Meteorites and Their Genetic Relationships, ed. A. M. Davis, 1,1

Kurucz, R. 1993, ATLAS9 Stellar Atmosphere Programs and 2 km/s grid. Kurucz CD-ROM No. 13. Cambridge, Mass.: Smithsonian Astrophysical Observatory, 1993, 13

Kurucz, R. L. 2005, Mem. Soc. Astron. It. Suppl., 8, 189

Kurucz, R. L. 2006, ArXiv e-prints [arXiv:astro-ph/0605029]

Kurucz, R. L., Furenlid, I., Brault, J., \& Testerman, L. 1984, Solar flux atlas from 296 to $1300 \mathrm{~nm}$

Lambert, D. L. 1968, MNRAS, 138, 143

Lambert, D. L. 1978, MNRAS, 182, 249

Lambert, D. L. 1993, Phys. Scr. Vol. T, 47, 186

Lambert, D. L., Mallia, E. A., \& Smith, G. 1972, Sol. Phys., 26, 250

Laming, J. M. 2015, Liv. Rev. Sol. Phys., 12, 2

Laming, J. M., Heber, V. S., Burnett, D. S., et al. 2017, ApJ, 851, L12

Landi, E., \& Testa, P. 2015, ApJ, 800, 110
Landi, E., Feldman, U., \& Doschek, G. A. 2007, ApJ, 659, 743

Lawler, J. E., Wickliffe, M. E., den Hartog, E. A., \& Sneden, C. 2001, ApJ, 563, 1075

Lawler, J. E., Guzman, A., Wood, M. P., Sneden, C., \& Cowan, J. J. 2013, ApJS, 205, 11

Lawler, J. E., Wood, M. P., Den Hartog, E. A., et al. 2014, ApJS, 215, 20

Lawler, J. E., Sneden, C., \& Cowan, J. J. 2015, ApJS, 220, 13

Lawler, J. E., Sneden, C., Nave, G., et al. 2017, ApJS, 228, 10

Lawler, J. E., Hala, C., Sneden, G., et al. 2019, ApJS, 241, 21

Leenaarts, J., \& Carlsson, M. 2009, in The Second Hinode Science Meeting, eds. B. Lites, M. Cheung, T. Magara, J. Mariska, \& K. Reeves, ASP Conf. Ser., 415,87

Lellouch, E., Bézard, B., Fouchet, T., et al. 2001, A\&A, 370, 610

Li, G., Gordon, I. E., Rothman, L. S., et al. 2015, ApJS, 216, 15

Li, W., Hartman, H., Wang, K., \& Jönsson, P. 2020, A\&A, 643, A156

Li, W., Amarsi, A. M., Papoulia, A., Ekman, J., \& Jönsson, P. 2021, MNRAS, 502,3780

Liang, G. Y., Whiteford, A. D., \& Badnell, N. R. 2009, J. Phys. B At. Mol. Phys., 42, 225002

Lind, K., Asplund, M., \& Barklem, P. S. 2009, A\&A, 503, 541

Lind, K., Asplund, M., Barklem, P. S., \& Belyaev, A. K. 2011, A\&A, 528, A103

Lind, K., Melendez, J., Asplund, M., Collet, R., \& Magic, Z. 2013, A\&A, 554, A96

Lind, K., Amarsi, A. M., Asplund, M., et al. 2017, MNRAS, 468, 4311

Linsky, J. L., Draine, B. T., Moos, H. W., et al. 2006, ApJ, 647, 1106

Liu, Y. P., Gao, C., Zeng, J. L., Yuan, J. M., \& Shi, J. R. 2014, ApJS, 211, 30

Ljung, G., Nilsson, H., Asplund, M., \& Johansson, S. 2006, A\&A, 456, 1181

Lodders, K. 2003, ApJ, 591, 1220

Lodders, K. 2008, ApJ, 674, 607

Lodders, K. 2019, ArXiv e-prints [arXiv:1912.00844]

Lodders, K., Palme, H., \& Gail, H. P. 2009, Landolt Börnstein, 44

Ludwig, H.-G., Freytag, B., \& Steffen, M. 1999, A\&A, 346, 111

Lyons, J. R., Gharib-Nezhad, E., \& Ayres, T. R. 2018, Nat. Commun., 9, 908

Maas, Z. G., Pilachowski, C. A., \& Hinkle, K. 2016, AJ, 152, 196

Magic, Z., Collet, R., Asplund, M., et al. 2013, A\&A, 557, A26

Mahaffy, P. R., Donahue, T. M., Atreya, S. K., Owen, T. C., \& Niemann, H. B. 1998, Space Sci. Rev., 84, 251

Maiorca, E., Uitenbroek, H., Uttenthaler, S., et al. 2014, ApJ, 788, 149

Malcheva, G., Engström, L., Lundberg, H., et al. 2015, MNRAS, 450, 223

Marty, B., Zimmermann, L., Burnard, P. G., et al. 2010, Geochim. Cosmochim. Acta, 74, 340

Mashonkina, L., Ryabchikova, T., Ryabtsev, A., \& Kildiyarova, R. 2009, A\&A, 495, 297

Mashonkina, L., Gehren, T., Shi, J.-R., Korn, A. J., \& Grupp, F. 2011, A\&A, 528, A87

Mashonkina, L., Sitnova, T., \& Belyaev, A. K. 2017, A\&A, 605, A53

Masseron, T., Plez, B., Van Eck, S., et al. 2014, A\&A, 571, A47

McKeegan, K. D., Kallio, A. P. A., Heber, V. S., et al. 2011, Science, 332, 1528

Meija, J., Coplen, T. B., Berglund, M., et al. 2016a, Pure Appl. Chem., 88, 265

Meija, J., Coplen, T. B., Berglund, M., et al. 2016b, Pure Appl. Chem., 88, 293

Meléndez, M., Bautista, M. A., \& Badnell, N. R. 2007, A\&A, 469, 1203

Meléndez, J., Asplund, M., Gustafsson, B., \& Yong, D. 2009, ApJ, 704, L66

Meshik, A., Hohenberg, C., Pravdivtseva, O., \& Burnett, D. 2014, Geochim. Cosmochim. Acta, 127, 326

Meshik, A., Pravdivtseva, O., \& Burnett, D. 2020, Geochim. Cosmochim. Acta, 276, 289

Mihalas, D., Dappen, W., \& Hummer, D. G. 1988, ApJ, 331, 815

Mondet, G., Blancard, C., Cossé, P., \& Faussurier, G. 2015, ApJS, 220, 2

Mott, A., Steffen, M., Caffau, E., Spada, F., \& Strassmeier, K. G. 2017, A\&A, 604, A44

Mott, A., Steffen, M., Caffau, E., \& Strassmeier, K. G. 2020, A\&A, 638, A58

Muthsam, H. J., Kupka, F., Löw-Baselli, B., et al. 2010, New Astron., 15, 460

Nagayama, T., Bailey, J. E., Loisel, G. P., et al. 2019, Phys. Rev. Lett., 122, 235001

Nahar, S. N., \& Pradhan, A. K. 2016, Phys. Rev. Lett., 116, 235003

Neckel, H. 1999, Sol. Phys., 184, 421

Nieva, M.-F., \& Przybilla, N. 2012, A\&A, 539, A143

Nissen, P. E., \& Gustafsson, B. 2018, A\&ARv, 26, 6

Nordlander, T., \& Lind, K. 2017, A\&A, 607, A75

Nordlund, A. 1982, A\&A, 107, 1

Nordlund, A., \& Dravins, D. 1990, A\&A, 228, 155

Nordlund, A., \& Galsgaard, K. 1995, A 3D MHD code for Parallel Computers, Tech. rep. (Niels Bohr Institute, University of Copenhagen)

Nordlund, A., Stein, R. F., \& Asplund, M. 2009, Liv. Rev. Sol. Phys., 6, 2

O’Brian, T. R., \& Lawler, J. E. 1991, Phys. Lett. A, 152, 407

O'Brian, T. R., Wickliffe, M. E., Lawler, J. E., Whaling, W., \& Brault, J. W. 1991, J. Opt. Soc. Am. B Opt. Phys., 8, 1185

Osorio, Y., Barklem, P. S., Lind, K., et al. 2015, A\&A, 579, A53 
Osorio, Y., Lind, K., Barklem, P. S., Allende Prieto, C., \& Zatsarinny, O. 2019, A\&A, 623, A103

Osorio, Y., Allende Prieto, C., Hubeny, I., Mészáros, S., \& Shetrone, M. 2020, A\&A, 637, A80

Owen, T., Mahaffy, P. R., Niemann, H. B., Atreya, S., \& Wong, M. 2001, ApJ, 553, L77

Palme, H., \& Hezel, D. C. 2011, Meteorit. Planet. Sci. Suppl., 74, 5302

Palme, H., Lodders, K., \& Jones, A. 2014, in Solar System Abundances of the Elements, ed. A. M. Davis, 2, 15

Payne, C. H. 1925, PhD Thesis, Radcliffe College, USA

Pehlivan Rhodin, A., Hartman, H., Nilsson, H., \& Jönsson, P. 2017a, A\&A, 598, A102

Pehlivan Rhodin, A., Belmonte, M. T., Engström, L., et al. 2017b, MNRAS, 472 3337

Pehlivan Rhodin, A., Hartmann, H., Nilsson, H., \& Jönsson, P. 2021, A\&A, submitted

Pereira, T. M. D., Asplund, M., \& Kiselman, D. 2009, A\&A, 508, 1403

Pereira, T. M. D., Asplund, M., Collet, R., et al. 2013, A\&A, 554, A118

Pinsonneault, M. H., \& Delahaye, F. 2009, ApJ, 704, 1174

Piskunov, N. E., Kupka, F., Ryabchikova, T. A., Weiss, W. W., \& Jeffery, C. S. 1995, A\&AS, 112, 525

Prodanović, T., Steigman, G., \& Fields, B. D. 2010, MNRAS, 406, 1108

Prša, A., Harmanec, P., Torres, G., et al. 2016, AJ, 152, 41

Ramírez, I., Asplund, M., Baumann, P., Meléndez, J., \& Bensby, T. 2010, A\&A, 521, A33

Reames, D. V. 2014, Sol. Phys., 289, 977

Reames, D. V. 2020, Space Sci. Rev., 216, 20

Reggiani, H., Amarsi, A. M., Lind, K., et al. 2019, A\&A, 627, A177

Reifarth, R., Heil, M., Käppeler, F., et al. 2002, Phys. Rev. C, 66, 064603

Reiners, A., Mrotzek, N., Lemke, U., Hinrichs, J., \& Reinsch, K. 2016, A\&A, 587, A65

Reisenfeld, D. B., Wiens, R. C., Barraclough, B. L., et al. 2013, Space Sci. Rev., 175,125

Rempel, M. 2014, ApJ, 789, 132

Rogers, F. J., \& Nayfonov, A. 2002, ApJ, 576, 1064

Ross, J. E., \& Aller, L. H. 1976, Science, 191, 1223

Ruffoni, M. P., Allende Prieto, C., Nave, G., \& Pickering, J. C. 2013, ApJ, 779, 17

Russell, H. N. 1929, ApJ, 70, 11

Rutten, R. J. 2003, Radiative Transfer in Stellar Atmospheres, 8th edn. (Utrecht University)

Rutten, R. J., \& Kostik, R. I. 1982, A\&A, 115, 104

Saloman, E. B., \& Kramida, A. 2017, ApJS, 231, 18

Scott, E. R. D. 2007, Ann. Rev. Earth Planet. Sci., 35, 577

Scott, P. C., Asplund, M., Grevesse, N., \& Sauval, A. J. 2006, A\&A, 456 675

Scott, P., Asplund, M., Grevesse, N., Bergemann, M., \& Sauval, A. J. 2015a, A\&A, 573, A26

Scott, P., Grevesse, N., Asplund, M., et al. 2015b, A\&A, 573, A25

Seaton, M. J. 1987, J. Phys. B, 20, 6363

Serenelli, A. M., Basu, S., Ferguson, J. W., \& Asplund, M. 2009, ApJ, 705, L123

Serenelli, A., Scott, P., Villante, F. L., et al. 2016, MNRAS, 463, 2

Shchukina, N., \& Trujillo Bueno, J. 2015, A\&A, 579, A112

Shchukina, N., Sukhorukov, A., \& Trujillo Bueno, J. 2016, A\&A, 586, A145

Shi, J. R., Gehren, T., Zeng, J. L., Mashonkina, L., \& Zhao, G. 2014, ApJ, 782 80

Short, C. I., \& Hauschildt, P. H. 2009, ApJ, 691, 1634

Sneden, C., Lawler, J. E., Cowan, J. J., Ivans, I. I., \& Den Hartog, E. A. 2009, ApJS, 182, 80

Sonoi, T., Samadi, R., Belkacem, K., et al. 2015, A\&A, 583, A112

Steenbock, W., \& Holweger, H. 1984, A\&A, 130, 319

Steffen, M., Caffau, E., \& Ludwig, H.-G. 2013, Mem. Soc. Astron. It. Suppl., 24, 37
Steffen, M., Prakapavičius, D., Caffau, E., et al. 2015, A\&A, 583, A57

Stein, R. F., \& Nordlund, A. 1998, ApJ, 499, 914

Stenflo, J. O. 2015, A\&A, 573, A74

Suess, H. E. 1947a, Zeitschrift Naturforschung Teil A, 2, 311

Suess, H. E. 1947b, Zeitschrift Naturforschung Teil A, 2, 604

Suess, H. E., \& Urey, H. C. 1956, Rev. Mod. Phys., 28, 53

Tachiev, G. I., \& Froese Fischer, C. 2002, A\&A, 385, 716

Takeda, Y. 1994, PASJ, 46, 53

Takeda, Y. 2021, Astron. Nachr., 342, 515

Takeda, Y., Kato, K.-I., Watanabe, Y., \& Sadakane, K. 1996, PASJ, 48, 511

Takeda, Y., Hashimoto, O., Taguchi, H., et al. 2005, PASJ, 57, 751

Tan, K., Shi, J., \& Zhao, G. 2010, ApJ, 713, 458

Théado, S., \& Vauclair, S. 2012, ApJ, 744, 123

Thoul, A. A., Bahcall, J. N., \& Loeb, A. 1994, ApJ, 421, 828

Trampedach, R., Däppen, W., \& Baturin, V. A. 2006, ApJ, 646, 560

Trampedach, R., Asplund, M., Collet, R., Nordlund, A., \& Stein, R. F. 2013, ApJ, 769, 18

Tucci Maia, M., Meléndez, J., Castro, M., et al. 2015, A\&A, 576, L10

Unsöld, A. 1948, ZAp, 24, 306

Urey, H. C. 1952, Phys. Rev., 88, 248

van Kooten, E. M. M. E., Moynier, F., \& Agranier, A. 2019, Proc. Natl. Acad. Sci., 116, 18860

Vinyoles, N., Serenelli, A. M., Villante, F. L., et al. 2017, ApJ, 835, 202

Vitas, N., Vince, I., Lugaro, M., et al. 2008, MNRAS, 384, 370

Vögler, A., \& Schüssler, M. 2007, A\&A, 465, L43

Vögler, A., Shelyag, S., Schüssler, M., et al. 2005, A\&A, 429, 335

von Steiger, R., \& Zurbuchen, T. H. 2016, ApJ, 816, 13

Vorontsov, S. V., Baturin, V. A., Ayukov, S. V., \& Gryaznov, V. K. 2013, MNRAS, 430, 1636

Wallace, L., Hinkle, K., \& Livingston, W. C. 2001, Sunspot umbral spectra in the region 4000 to $8640 \mathrm{~cm}(-1)$ (1.16 to 2.50 [microns])

Wang, K., Bartschat, K., \& Zatsarinny, O. 2018, ApJ, 867, 63

Wang, H. S., Lineweaver, C. H., \& Ireland, T. R. 2019, Icarus, 328, 287

Wang, E. X., Nordlander, T., Asplund, M., et al. 2021, MNRAS, 500, 2159

Wasson, J. T., \& Kallemeyn, G. W. 1988, Phil. Trans. R. Soc. London, Ser. A, 325,535

Weinberg, D. H. 2017, ApJ, 851, 25

Wiens, R. C., Reisenfeld, D. B., Olinger, C., et al. 2013, Space Sci. Rev., 175, 93

Wisshak, K., Voss, F., Käppeler, F., Kazakov, L., \& Reffo, G. 1998, Phys. Rev. C, 57,391

Wittenmyer, R. A., Wang, S., Horner, J., et al. 2020, MNRAS, 492, 377

Wood, M. P., Lawler, J. E., Sneden, C., \& Cowan, J. J. 2013, ApJS, 208, 27

Wood, M. P., Lawler, J. E., Den Hartog, E. A., Sneden, C., \& Cowan, J. J. 2014a, ApJS, 214, 18

Wood, M. P., Lawler, J. E., Sneden, C., \& Cowan, J. J. 2014b, ApJS, 211, 20

Wood, B. E., Müller, H.-R., \& Möbius, E. 2019, ApJ, 881, 55

Yakovleva, S. A., Barklem, P. S., \& Belyaev, A. K. 2018, MNRAS, 473, 3810

Yakovleva, S. A., Belyaev, A. K., \& Kraemer, W. P. 2019, MNRAS, 483, 5105

Yana Galarza, J., Meléndez, J., Karakas, A. I., Asplund, M., \& Lorenzo-Oliveira, D. 2021, MNRAS, 502, L104

Yang, W. 2019, ApJ, 873, 18

Young, P. R. 2005, A\&A, 439, 361

Young, P. R. 2018, ApJ, 855, 15

Zatsarinny, O. 2006, Comput. Phys. Commun., 174, 273

Zatsarinny, O., \& Bartschat, K. 2013, J. Phys. B, 46, 112001

Zatsarinny, O., Parker, H., \& Bartschat, K. 2019a, Phys. Rev. A, 99, 012706

Zatsarinny, O., Bartschat, K., Fernandez-Menchero, L., \& Tayal, S. S. 2019b, Phys. Rev. A, 99, 023430

Zhang, H. L., \& Pradhan, A. K. 1995, A\&A, 293

Zhang, Q.-S., Li, Y., \& Christensen-Dalsgaard, J. 2019, ApJ, 881, 103

Zhou, Y., Asplund, M., \& Collet, R. 2019, ApJ, 880, 13

Zink, J. K., Christiansen, J. L., \& Hansen, B. M. S. 2019, MNRAS, 483, 4479 


\section{Appendix A: Line lists for $\mathrm{Na}, \mathrm{Mg}, \mathrm{K}, \mathrm{Ca}$, and $\mathrm{Fe}$}

Table A.1. Line list for the $\mathrm{Na}, \mathrm{Mg}, \mathrm{K}$, and $\mathrm{Ca}$ lines used for 3D non-LTE spectral line formation calculations to derive the solar photospheric abundances.

\begin{tabular}{|c|c|c|c|c|c|c|c|c|c|c|c|c|}
\hline \multirow[t]{2}{*}{$\lambda_{\text {air }} / \mathrm{nm}$} & \multirow[t]{2}{*}{$E_{\text {lower }} / \mathrm{eV}$} & \multirow[t]{2}{*}{$E_{\text {upper }} / \mathrm{eV}$} & \multirow[t]{2}{*}{$\log g f$} & \multirow[t]{2}{*}{$W / \mathrm{pm}$} & \multicolumn{4}{|c|}{ Non-LTE } & \multicolumn{4}{|c|}{ LTE } \\
\hline & & & & & $3 \mathrm{D}$ & $\langle 3 \mathrm{D}\rangle$ & MARCS & HM & $3 \mathrm{D}$ & $\langle 3 \mathrm{D}\rangle$ & MARCS & HM \\
\hline \multicolumn{13}{|c|}{$\mathrm{Na}$ I } \\
\hline 475.18 & 2.10443 & 4.71289 & -2.077 & 1.14 & 6.223 & 6.225 & 6.199 & 6.243 & 6.250 & 6.259 & 6.231 & 6.280 \\
\hline 514.88 & 2.10230 & 4.50963 & -2.044 & 1.32 & 6.231 & 6.235 & 6.206 & 6.251 & 6.259 & 6.271 & 6.239 & 6.288 \\
\hline 615.42 & 2.10230 & 4.11636 & -1.547 & 3.98 & 6.250 & 6.255 & 6.215 & 6.266 & 6.294 & 6.307 & 6.262 & 6.320 \\
\hline 616.07 & 2.10443 & 4.11636 & -1.246 & 5.83 & 6.178 & 6.179 & 6.135 & 6.190 & 6.229 & 6.240 & 6.193 & 6.256 \\
\hline 1074.64 & 3.19135 & 4.34476 & -1.294 & 1.33 & 6.221 & 6.221 & 6.188 & 6.223 & 6.226 & 6.227 & 6.194 & 6.231 \\
\hline \multicolumn{13}{|c|}{ Mg I } \\
\hline 631.87 & 5.10783 & 7.06946 & -2.020 & 4.13 & 7.546 & 7.546 & 7.509 & 7.559 & 7.545 & 7.547 & 7.509 & 7.559 \\
\hline 631.92 & 5.10783 & 7.06930 & -2.242 & 2.60 & 7.549 & 7.547 & 7.516 & 7.558 & 7.548 & 7.547 & 7.516 & 7.558 \\
\hline 871.27 & 5.93195 & 7.35459 & -1.152 & 6.80 & 7.565 & 7.560 & 7.517 & 7.566 & 7.566 & 7.561 & 7.518 & 7.568 \\
\hline 871.78 & 5.93279 & 7.35459 & -0.930 & 10.00 & 7.581 & 7.575 & 7.524 & 7.584 & 7.583 & 7.577 & 7.526 & 7.587 \\
\hline 892.36 & 5.39373 & 6.78275 & -1.679 & 6.33 & 7.608 & 7.605 & 7.562 & 7.614 & 7.607 & 7.605 & 7.562 & 7.614 \\
\hline 998.32 & 5.93154 & 7.17313 & -2.177 & 1.00 & 7.558 & 7.553 & 7.525 & 7.556 & 7.559 & 7.554 & 7.526 & 7.558 \\
\hline 1031.25 & 6.11821 & 7.32015 & -1.718 & 1.83 & 7.515 & 7.508 & 7.479 & 7.513 & 7.515 & 7.509 & 7.479 & 7.513 \\
\hline 1587.95 & 5.94592 & 6.72648 & -1.226 & 16.80 & 7.570 & 7.565 & 7.517 & 7.594 & 7.571 & 7.566 & 7.518 & 7.595 \\
\hline \multicolumn{13}{|c|}{ Mg II } \\
\hline 787.70 & 9.99554 & 11.56910 & 0.389 & 1.90 & 7.540 & 7.542 & 7.526 & 7.572 & 7.568 & 7.570 & 7.542 & 7.606 \\
\hline 789.64 & 9.99933 & 11.56904 & 0.645 & 3.00 & 7.635 & 7.655 & 7.640 & 7.681 & 7.682 & 7.702 & 7.669 & 7.731 \\
\hline 921.82 & 8.65471 & 9.99933 & 0.269 & 7.40 & 7.531 & 7.501 & 7.481 & 7.504 & 7.642 & 7.592 & 7.559 & 7.607 \\
\hline 924.43 & 8.65471 & 9.99554 & -0.034 & 5.15 & 7.489 & 7.465 & 7.447 & 7.478 & 7.567 & 7.535 & 7.505 & 7.554 \\
\hline 1009.22 & 11.62968 & 12.85786 & 1.283 & 1.33 & 7.495 & 7.541 & 7.523 & 7.598 & 7.490 & 7.536 & 7.518 & 7.596 \\
\hline 1091.42 & 8.86365 & 9.99933 & 0.036 & 5.22 & 7.427 & 7.401 & 7.388 & 7.419 & 7.518 & 7.481 & 7.451 & 7.509 \\
\hline \multicolumn{13}{|c|}{ K I } \\
\hline 693.88 & 1.61711 & 3.40345 & -1.145 & 0.43 & 5.045 & 5.044 & 5.010 & 5.050 & 5.071 & 5.081 & 5.046 & 5.087 \\
\hline 1176.97 & 1.61711 & 2.67025 & -0.481 & 3.50 & 5.082 & 5.070 & 5.029 & 5.079 & 5.135 & 5.144 & 5.102 & 5.153 \\
\hline 1252.22 & 1.61711 & 2.60696 & -0.128 & 7.60 & 5.093 & 5.075 & 5.025 & 5.090 & 5.157 & 5.163 & 5.110 & 5.178 \\
\hline \multicolumn{13}{|c|}{$\mathrm{Ca}$} \\
\hline 451.23 & 2.52568 & 5.27263 & -1.900 & 2.20 & 6.296 & 6.304 & 6.272 & 6.327 & 6.285 & 6.304 & 6.270 & 6.327 \\
\hline 526.04 & 2.52126 & 4.87755 & -1.719 & 3.00 & 6.265 & 6.271 & 6.230 & 6.290 & 6.253 & 6.272 & 6.230 & 6.292 \\
\hline 586.76 & 2.93251 & 5.04497 & -1.570 & 2.30 & 6.278 & 6.284 & 6.244 & 6.298 & 6.269 & 6.285 & 6.244 & 6.300 \\
\hline 616.38 & 2.52126 & 4.53221 & -1.286 & 6.20 & 6.295 & 6.331 & 6.282 & 6.350 & 6.286 & 6.339 & 6.290 & 6.362 \\
\hline 616.64 & 2.52126 & 4.53134 & -1.142 & 7.13 & 6.281 & 6.271 & 6.210 & 6.294 & 6.275 & 6.284 & 6.221 & 6.309 \\
\hline $616.93^{(a)}$ & 2.52433 & 4.53347 & -0.308 & 21.66 & 6.293 & 6.259 & 6.185 & 6.287 & 6.304 & 6.295 & 6.215 & 6.325 \\
\hline 645.56 & 2.52299 & 4.44302 & -1.340 & 5.65 & 6.319 & 6.309 & 6.261 & 6.326 & 6.311 & 6.322 & 6.272 & 6.340 \\
\hline 647.17 & 2.52568 & 4.44095 & -0.686 & 9.30 & 6.297 & 6.241 & 6.177 & 6.263 & 6.317 & 6.294 & 6.224 & 6.320 \\
\hline 649.96 & 2.52299 & 4.43001 & -0.818 & 8.75 & 6.334 & 6.286 & 6.225 & 6.307 & 6.348 & 6.330 & 6.264 & 6.355 \\
\hline \multicolumn{13}{|c|}{$\mathrm{Ca}$ II } \\
\hline 500.15 & 7.50514 & 9.98340 & -0.507 & 1.35 & 6.231 & 6.235 & 6.238 & 6.290 & 6.237 & 6.241 & 6.243 & 6.299 \\
\hline 645.69 & 8.43798 & 10.35764 & 0.412 & 1.85 & 6.296 & 6.291 & 6.269 & 6.329 & 6.296 & 6.293 & 6.269 & 6.331 \\
\hline 732.39 & 0.00000 & 1.69241 & -7.536 & 1.00 & 6.372 & 6.373 & 6.344 & 6.391 & 6.372 & 6.373 & 6.344 & 6.391 \\
\hline 824.88 & 7.51484 & 9.01749 & 0.556 & 6.70 & 6.302 & 6.261 & 6.228 & 6.267 & 6.364 & 6.324 & 6.279 & 6.339 \\
\hline 825.47 & 7.51484 & 9.01641 & -0.398 & 1.80 & 6.296 & 6.284 & 6.270 & 6.303 & 6.322 & 6.310 & 6.291 & 6.333 \\
\hline
\end{tabular}

Notes. ${ }^{(a)}$ Doublet consisting of the $616.9042 \mathrm{~nm}$ and $616.9563 \mathrm{~nm}$ components. The quoted $g f$-value and equivalent width are the combined values. 
Table A.2. Line list for the Fe lines used for 3D non-LTE spectral line formation calculations to derive the solar photospheric abundances.

\begin{tabular}{|c|c|c|c|c|c|c|c|c|c|c|c|c|}
\hline \multirow[t]{2}{*}{$\lambda_{\text {air }} / \mathrm{nm}$} & \multirow[t]{2}{*}{$E_{\text {lower }} / \mathrm{eV}$} & \multirow[t]{2}{*}{$E_{\text {upper }} / \mathrm{eV}$} & \multirow[t]{2}{*}{$\log g f$} & \multirow[t]{2}{*}{$W / \mathrm{pm}$} & \multicolumn{4}{|c|}{ Non-LTE } & \multicolumn{4}{|c|}{ LTE } \\
\hline & & & & & $3 \mathrm{D}$ & $\langle 3 \mathrm{D}\rangle$ & MARCS & HM & $3 \mathrm{D}$ & $\langle 3 \mathrm{D}\rangle$ & MARCS & $\mathrm{HM}$ \\
\hline \multicolumn{13}{|c|}{$\mathrm{Fe} I$} \\
\hline 444.55 & 0.08729 & 2.87550 & -5.412 & 3.80 & 7.455 & 7.496 & 7.453 & 7.542 & 7.424 & 7.511 & 7.462 & 7.558 \\
\hline 457.42 & 3.21119 & 5.92093 & -2.350 & 3.87 & 7.451 & 7.448 & 7.408 & 7.475 & 7.440 & 7.450 & 7.408 & 7.479 \\
\hline 524.70 & 0.08729 & 2.44956 & -4.961 & 6.40 & 7.483 & 7.477 & 7.421 & 7.523 & 7.434 & 7.498 & 7.436 & 7.550 \\
\hline 537.96 & 3.69460 & 5.99868 & -1.420 & 6.17 & 7.433 & 7.383 & 7.329 & 7.413 & 7.429 & 7.399 & 7.340 & 7.433 \\
\hline 548.31 & 4.15435 & 6.41493 & -1.390 & 4.37 & 7.417 & 7.399 & 7.353 & 7.421 & 7.407 & 7.401 & 7.352 & 7.426 \\
\hline 553.85 & 4.21758 & 6.45554 & -1.540 & 3.73 & 7.488 & 7.477 & 7.436 & 7.495 & 7.480 & 7.478 & 7.435 & 7.498 \\
\hline 560.02 & 4.26045 & 6.47375 & -1.420 & 3.65 & 7.402 & 7.386 & 7.343 & 7.405 & 7.388 & 7.385 & 7.339 & 7.407 \\
\hline 561.86 & 4.20888 & 6.41493 & -1.250 & 5.00 & 7.444 & 7.418 & 7.369 & 7.440 & 7.432 & 7.421 & 7.368 & 7.446 \\
\hline 566.13 & 4.28435 & 6.47375 & -1.756 & 2.22 & 7.437 & 7.429 & 7.393 & 7.444 & 7.424 & 7.427 & 7.389 & 7.445 \\
\hline 570.55 & 4.30128 & 6.47375 & -1.355 & 3.96 & 7.428 & 7.411 & 7.367 & 7.430 & 7.418 & 7.413 & 7.366 & 7.434 \\
\hline 577.51 & 4.22036 & 6.36665 & -1.080 & 6.19 & 7.486 & 7.449 & 7.396 & 7.473 & 7.481 & 7.459 & 7.401 & 7.486 \\
\hline 577.85 & 2.58811 & 4.73314 & -3.440 & 2.04 & 7.430 & 7.446 & 7.406 & 7.467 & 7.416 & 7.451 & 7.409 & 7.474 \\
\hline 578.47 & 3.39651 & 5.53924 & -2.532 & 2.58 & 7.443 & 7.444 & 7.405 & 7.462 & 7.429 & 7.445 & 7.403 & 7.465 \\
\hline 585.51 & 4.60759 & 6.72456 & -1.478 & 2.20 & 7.441 & 7.432 & 7.395 & 7.445 & 7.430 & 7.430 & 7.392 & 7.446 \\
\hline 595.67 & 0.85900 & 2.93985 & -4.552 & 5.02 & 7.451 & 7.467 & 7.419 & 7.500 & 7.424 & 7.483 & 7.431 & 7.522 \\
\hline 615.16 & 2.17595 & 4.19086 & -3.282 & 4.88 & 7.459 & 7.455 & 7.409 & 7.481 & 7.443 & 7.466 & 7.417 & 7.494 \\
\hline 624.06 & 2.22271 & 4.20888 & -3.287 & 4.76 & 7.478 & 7.477 & 7.432 & 7.503 & 7.465 & 7.488 & 7.441 & 7.519 \\
\hline 631.15 & 2.83159 & 4.79547 & -3.141 & 2.66 & 7.492 & 7.502 & 7.463 & 7.524 & 7.481 & 7.509 & 7.467 & 7.532 \\
\hline 649.89 & 0.95816 & 2.86539 & -4.695 & 4.16 & 7.469 & 7.499 & 7.455 & 7.536 & 7.445 & 7.517 & 7.466 & 7.555 \\
\hline 651.84 & 2.83159 & 4.73314 & -2.448 & 5.72 & 7.405 & 7.378 & 7.325 & 7.405 & 7.392 & 7.392 & 7.334 & 7.421 \\
\hline 657.42 & 0.99011 & 2.87550 & -5.010 & 2.54 & 7.465 & 7.517 & 7.472 & 7.549 & 7.442 & 7.532 & 7.482 & 7.565 \\
\hline 669.91 & 4.59311 & 6.44335 & -2.101 & 0.78 & 7.487 & 7.481 & 7.448 & 7.488 & 7.476 & 7.480 & 7.445 & 7.490 \\
\hline 679.33 & 4.07581 & 5.90041 & -2.326 & 1.25 & 7.445 & 7.443 & 7.408 & 7.453 & 7.434 & 7.444 & 7.407 & 7.456 \\
\hline 683.70 & 4.59311 & 6.40604 & -1.687 & 1.77 & 7.486 & 7.474 & 7.440 & 7.482 & 7.475 & 7.475 & 7.438 & 7.485 \\
\hline 684.37 & 4.54851 & 6.35967 & -0.730 & 6.50 & 7.409 & 7.362 & 7.304 & 7.380 & 7.401 & 7.373 & 7.309 & 7.396 \\
\hline 685.48 & 4.59311 & 6.40133 & -1.926 & 1.29 & 7.434 & 7.439 & 7.395 & 7.451 & 7.423 & 7.438 & 7.391 & 7.451 \\
\hline 685.81 & 4.60759 & 6.41493 & -0.900 & 5.50 & 7.464 & 7.431 & 7.380 & 7.445 & 7.456 & 7.437 & 7.382 & 7.455 \\
\hline 699.99 & 4.10337 & 5.87412 & -1.380 & 5.79 & 7.482 & 7.456 & 7.405 & 7.472 & 7.477 & 7.466 & 7.412 & 7.486 \\
\hline 700.80 & 4.17770 & 5.94640 & -1.770 & 2.95 & 7.435 & 7.426 & 7.386 & 7.438 & 7.425 & 7.429 & 7.386 & 7.443 \\
\hline 721.97 & 4.07581 & 5.79265 & -1.430 & 4.90 & 7.399 & 7.366 & 7.318 & 7.381 & 7.393 & 7.379 & 7.326 & 7.397 \\
\hline 740.17 & 4.18636 & 5.86098 & -1.500 & 4.16 & 7.423 & 7.399 & 7.355 & 7.411 & 7.415 & 7.408 & 7.361 & 7.423 \\
\hline 744.30 & 4.18636 & 5.85168 & -1.640 & 3.59 & 7.450 & 7.431 & 7.390 & 7.441 & 7.439 & 7.436 & 7.392 & 7.449 \\
\hline 791.29 & 0.85900 & 2.42543 & -4.848 & 4.57 & 7.480 & 7.517 & 7.470 & 7.552 & 7.452 & 7.540 & 7.484 & 7.575 \\
\hline 829.35 & 3.30092 & 4.79547 & -2.203 & 5.85 & 7.486 & 7.461 & 7.416 & 7.480 & 7.475 & 7.475 & 7.425 & 7.495 \\
\hline 857.18 & 5.00952 & 6.45554 & -1.110 & 3.17 & 7.499 & 7.489 & 7.452 & 7.495 & 7.501 & 7.496 & 7.457 & 7.505 \\
\hline 859.88 & 4.38646 & 5.82794 & -1.200 & 5.67 & 7.419 & 7.394 & 7.343 & 7.407 & 7.413 & 7.404 & 7.350 & 7.421 \\
\hline 887.60 & 5.02028 & 6.41674 & -1.050 & 3.55 & 7.502 & 7.489 & 7.451 & 7.495 & 7.504 & 7.497 & 7.457 & 7.506 \\
\hline 890.60 & 5.06378 & 6.45554 & -1.220 & 2.47 & 7.492 & 7.484 & 7.448 & 7.488 & 7.492 & 7.490 & 7.453 & 7.496 \\
\hline 910.36 & 4.17770 & 5.53924 & -2.190 & 1.92 & 7.481 & 7.479 & 7.444 & 7.487 & 7.468 & 7.479 & 7.441 & 7.489 \\
\hline 978.66 & 4.60759 & 5.87412 & -1.840 & 1.82 & 7.466 & 7.460 & 7.425 & 7.466 & 7.456 & 7.461 & 7.424 & 7.469 \\
\hline \multicolumn{13}{|c|}{ Fe II } \\
\hline 441.68 & 2.77846 & 5.58477 & -2.570 & 8.07 & 7.419 & 7.316 & 7.283 & 7.370 & 7.427 & 7.319 & 7.286 & 7.374 \\
\hline 450.83 & 2.85552 & 5.60489 & -2.420 & 8.85 & 7.488 & 7.382 & 7.346 & 7.434 & 7.498 & 7.386 & 7.350 & 7.440 \\
\hline 462.05 & 2.82812 & 5.51071 & -3.210 & 5.40 & 7.429 & 7.362 & 7.335 & 7.406 & 7.433 & 7.363 & 7.336 & 7.407 \\
\hline 465.70 & 2.89102 & 5.55261 & -3.600 & 3.60 & 7.435 & 7.395 & 7.381 & 7.434 & 7.437 & 7.396 & 7.381 & 7.434 \\
\hline 523.46 & 3.22131 & 5.58920 & -2.180 & 8.80 & 7.484 & 7.361 & 7.318 & 7.403 & 7.498 & 7.368 & 7.323 & 7.410 \\
\hline 526.48 & 3.23046 & 5.58477 & -3.130 & 4.62 & 7.496 & 7.438 & 7.413 & 7.469 & 7.499 & 7.439 & 7.414 & 7.470 \\
\hline 541.41 & 3.22131 & 5.51071 & -3.580 & 2.73 & 7.471 & 7.436 & 7.421 & 7.464 & 7.473 & 7.436 & 7.421 & 7.464 \\
\hline 643.27 & 2.89102 & 4.81790 & -3.570 & 4.30 & 7.492 & 7.434 & 7.409 & 7.454 & 7.494 & 7.434 & 7.408 & 7.453 \\
\hline 651.61 & 2.89102 & 4.79324 & -3.310 & 5.69 & 7.545 & 7.454 & 7.422 & 7.474 & 7.548 & 7.453 & 7.422 & 7.473 \\
\hline 722.24 & 3.88870 & 5.60489 & -3.260 & 1.87 & 7.455 & 7.419 & 7.406 & 7.436 & 7.457 & 7.420 & 7.407 & 7.436 \\
\hline 722.45 & 3.88919 & 5.60489 & -3.200 & 2.10 & 7.469 & 7.431 & 7.417 & 7.447 & 7.470 & 7.431 & 7.417 & 7.447 \\
\hline 751.58 & 3.90342 & 5.55261 & -3.390 & 1.47 & 7.447 & 7.414 & 7.403 & 7.429 & 7.448 & 7.414 & 7.403 & 7.430 \\
\hline 771.17 & 3.90342 & 5.51071 & -2.500 & 5.04 & 7.457 & 7.376 & 7.345 & 7.388 & 7.462 & 7.378 & 7.347 & 7.391 \\
\hline
\end{tabular}




\section{Appendix B: Isotopic abundances}

Table B.1. Representative isotopic abundance fractions and logarithmic abundances by number $\left(\log \epsilon_{\mathrm{H}} \equiv 12.00\right)$ at the time of birth of the Sun.

\begin{tabular}{|c|c|c|c|c|c|c|c|c|c|c|c|c|c|c|}
\hline $\mathrm{Z}$ & Elem. & A & fraction $\%$ & $\log \epsilon$ & $\mathrm{Z}$ & Elem. & A & fraction $\%$ & $\log \epsilon$ & $\mathrm{Z}$ & Elem. & A & fraction $\%$ & $\log \epsilon$ \\
\hline 1 & $\mathrm{H}$ & 1 & 99.998 & 12.00 & 24 & $\mathrm{Cr}$ & 50 & 4.345 & 4.32 & 41 & $\mathrm{Nb}$ & 93 & 100.000 & 1.53 \\
\hline 1 & $\mathrm{H}$ & 2 & 0.002 & 7.22 & 24 & $\mathrm{Cr}$ & 52 & 83.789 & 5.61 & 42 & Mo & 92 & 14.649 & 1.11 \\
\hline 2 & $\mathrm{He}$ & 3 & 0.017 & 7.20 & 24 & $\mathrm{Cr}$ & 53 & 9.501 & 4.66 & 42 & Mo & 94 & 9.187 & 0.91 \\
\hline 2 & $\mathrm{He}$ & 4 & 99.983 & 10.98 & 24 & $\mathrm{Cr}$ & 54 & 2.365 & 4.06 & 42 & Mo & 95 & 15.873 & 1.14 \\
\hline 3 & $\mathrm{Li}$ & 6 & 4.850 & 1.98 & 25 & $\mathrm{Mn}$ & 55 & 100.000 & 5.48 & 42 & Mo & 96 & 16.673 & 1.17 \\
\hline 3 & $\mathrm{Li}$ & 7 & 95.150 & 3.28 & 26 & $\mathrm{Fe}$ & 54 & 5.845 & 6.29 & 42 & Mo & 97 & 9.582 & 0.93 \\
\hline 4 & $\mathrm{Be}$ & 9 & 100.000 & 1.44 & 26 & $\mathrm{Fe}$ & 56 & 91.754 & 7.49 & 42 & Mo & 98 & 24.292 & 1.33 \\
\hline 5 & B & 10 & 19.650 & 2.06 & 26 & $\mathrm{Fe}$ & 57 & 2.119 & 5.85 & 42 & Mo & 100 & 9.744 & 0.93 \\
\hline 5 & B & 11 & 80.350 & 2.67 & 26 & $\mathrm{Fe}$ & 58 & 0.282 & 4.97 & 44 & $\mathrm{Ru}$ & 96 & 5.540 & 0.56 \\
\hline 6 & $\mathrm{C}$ & 12 & 98.893 & 8.52 & 27 & Co & 59 & 100.000 & 5.00 & 44 & $\mathrm{Ru}$ & 98 & 1.870 & 0.09 \\
\hline 6 & $\mathrm{C}$ & 13 & 1.107 & 6.57 & 28 & $\mathrm{Ni}$ & 58 & 68.077 & 6.10 & 44 & $\mathrm{Ru}$ & 99 & 12.760 & 0.92 \\
\hline 7 & $\mathrm{~N}$ & 14 & 99.775 & 7.89 & 28 & $\mathrm{Ni}$ & 60 & 26.223 & 5.68 & 44 & $\mathrm{Ru}$ & 100 & 12.600 & 0.91 \\
\hline 7 & $\mathrm{~N}$ & 15 & 0.225 & 5.25 & 28 & $\mathrm{Ni}$ & 61 & 1.140 & 4.32 & 44 & $\mathrm{Ru}$ & 101 & 17.060 & 1.05 \\
\hline 8 & $\mathrm{O}$ & 16 & 99.776 & 8.75 & 28 & $\mathrm{Ni}$ & 62 & 3.635 & 4.82 & 44 & $\mathrm{Ru}$ & 102 & 31.550 & 1.31 \\
\hline 8 & $\mathrm{O}$ & 17 & 0.036 & 5.31 & 28 & $\mathrm{Ni}$ & 64 & 0.926 & 4.23 & 44 & $\mathrm{Ru}$ & 104 & 18.620 & 1.08 \\
\hline 8 & $\mathrm{O}$ & 18 & 0.188 & 6.03 & 29 & $\mathrm{Cu}$ & 63 & 69.150 & 4.08 & 44 & $\mathrm{Rh}$ & 103 & 100.000 & 0.84 \\
\hline 9 & $\mathrm{~F}$ & 19 & 100.000 & 4.46 & 29 & $\mathrm{Cu}$ & 65 & 30.850 & 3.73 & 45 & $\mathrm{Pd}$ & 102 & 1.020 & -0.36 \\
\hline 10 & $\mathrm{Ne}$ & 20 & 92.810 & 8.09 & 30 & $\mathrm{Zn}$ & 64 & 49.170 & 4.32 & 46 & $\mathrm{Pd}$ & 104 & 11.140 & 0.68 \\
\hline 10 & $\mathrm{Ne}$ & 21 & 0.242 & 5.51 & 30 & $\mathrm{Zn}$ & 66 & 27.730 & 4.07 & 46 & $\mathrm{Pd}$ & 105 & 22.330 & 0.98 \\
\hline 10 & $\mathrm{Ne}$ & 22 & 6.948 & 6.97 & 30 & $\mathrm{Zn}$ & 67 & 4.040 & 3.23 & 46 & $\mathrm{Pd}$ & 106 & 27.330 & 1.07 \\
\hline 11 & $\mathrm{Na}$ & 23 & 100.000 & 6.28 & 30 & $\mathrm{Zn}$ & 68 & 18.450 & 3.89 & 46 & $\mathrm{Pd}$ & 108 & 26.460 & 1.06 \\
\hline 12 & $\mathrm{Mg}$ & 24 & 78.965 & 7.51 & 30 & $\mathrm{Zn}$ & 70 & 0.610 & 2.41 & 46 & $\mathrm{Pd}$ & 110 & 11.720 & 0.70 \\
\hline 12 & $\mathrm{Mg}$ & 25 & 10.011 & 6.61 & 31 & $\mathrm{Ga}$ & 69 & 60.108 & 2.86 & 46 & $\mathrm{Ag}$ & 107 & 51.839 & 0.74 \\
\hline 12 & $\mathrm{Mg}$ & 26 & 11.025 & 6.66 & 31 & $\mathrm{Ga}$ & 71 & 39.892 & 2.68 & 47 & $\mathrm{Ag}$ & 109 & 48.161 & 0.71 \\
\hline 13 & $\mathrm{Al}$ & 27 & 100.000 & 6.49 & 32 & $\mathrm{Ge}$ & 70 & 20.520 & 3.00 & 47 & $\mathrm{Cd}$ & 106 & 1.245 & -0.19 \\
\hline 14 & $\mathrm{Si}$ & 28 & 92.254 & 7.54 & 32 & $\mathrm{Ge}$ & 72 & 27.450 & 3.12 & 48 & $\mathrm{Cd}$ & 108 & 0.888 & -0.34 \\
\hline 14 & $\mathrm{Si}$ & 29 & 4.672 & 6.24 & 32 & $\mathrm{Ge}$ & 73 & 7.760 & 2.57 & 48 & $\mathrm{Cd}$ & 110 & 12.470 & 0.81 \\
\hline 14 & $\mathrm{Si}$ & 30 & 3.073 & 6.06 & 32 & $\mathrm{Ge}$ & 74 & 36.520 & 3.25 & 48 & $\mathrm{Cd}$ & 111 & 12.795 & 0.82 \\
\hline 15 & $\mathrm{P}$ & 31 & 100.000 & 5.47 & 32 & $\mathrm{Ge}$ & 76 & 7.750 & 2.57 & 48 & $\mathrm{Cd}$ & 112 & 24.109 & 1.10 \\
\hline 16 & $\mathrm{~S}$ & 32 & 94.850 & 7.16 & 33 & As & 75 & 100.000 & 2.35 & 48 & $\mathrm{Cd}$ & 113 & 12.227 & 0.80 \\
\hline 16 & $\mathrm{~S}$ & 33 & 0.763 & 5.07 & 34 & $\mathrm{Se}$ & 74 & 0.860 & 1.29 & 48 & $\mathrm{Cd}$ & 114 & 28.754 & 1.17 \\
\hline 16 & $\mathrm{~S}$ & 34 & 4.365 & 5.82 & 34 & $\mathrm{Se}$ & 76 & 9.230 & 2.32 & 48 & $\mathrm{Cd}$ & 116 & 7.512 & 0.59 \\
\hline 16 & $\mathrm{~S}$ & 36 & 0.016 & 3.38 & 34 & $\mathrm{Se}$ & 77 & 7.600 & 2.24 & 48 & In & 113 & 4.281 & -0.50 \\
\hline 17 & $\mathrm{Cl}$ & 35 & 75.800 & 5.25 & 34 & $\mathrm{Se}$ & 78 & 23.690 & 2.73 & 49 & In & 115 & 95.719 & 0.84 \\
\hline 17 & $\mathrm{Cl}$ & 37 & 24.200 & 4.76 & 34 & $\mathrm{Se}$ & 80 & 49.800 & 3.05 & 49 & Sn & 112 & 0.970 & 0.07 \\
\hline 18 & $\mathrm{Ar}$ & 36 & 84.281 & 6.37 & 34 & $\mathrm{Se}$ & 82 & 8.820 & 2.30 & 50 & $\mathrm{Sn}$ & 114 & 0.660 & 0.10 \\
\hline 18 & $\mathrm{Ar}$ & 38 & 15.695 & 5.64 & 35 & $\mathrm{Br}$ & 79 & 50.650 & 2.24 & 50 & $\mathrm{Sn}$ & 115 & 0.340 & 0.38 \\
\hline 18 & $\mathrm{Ar}$ & 40 & 0.024 & 2.83 & 35 & $\mathrm{Br}$ & 81 & 49.350 & 2.23 & 50 & $\mathrm{Sn}$ & 116 & 14.540 & 1.25 \\
\hline 19 & $\mathrm{~K}$ & 39 & 93.133 & 5.10 & 36 & $\mathrm{Kr}$ & 78 & 0.365 & 0.75 & 50 & Sn & 117 & 7.680 & 0.97 \\
\hline 19 & $\mathrm{~K}^{(*)}$ & 40 & 0.146 & 2.30 & 36 & $\mathrm{Kr}$ & 80 & 2.344 & 1.55 & 50 & $\mathrm{Sn}$ & 118 & 24.220 & 1.47 \\
\hline 19 & $\mathrm{~K}$ & 41 & 6.721 & 3.96 & 36 & $\mathrm{Kr}$ & 82 & 11.686 & 2.25 & 50 & Sn & 119 & 8.590 & 1.02 \\
\hline 20 & $\mathrm{Ca}$ & 40 & 96.941 & 6.35 & 36 & $\mathrm{Kr}$ & 83 & 11.573 & 2.25 & 50 & $\mathrm{Sn}$ & 120 & 32.580 & 1.60 \\
\hline 20 & $\mathrm{Ca}$ & 42 & 0.647 & 4.17 & 36 & $\mathrm{Kr}$ & 84 & 56.895 & 2.94 & 50 & $\mathrm{Sn}$ & 122 & 4.630 & 0.75 \\
\hline 20 & $\mathrm{Ca}$ & 43 & 0.135 & 3.49 & 36 & $\mathrm{Kr}$ & 86 & 17.137 & 2.42 & 50 & $\mathrm{Sn}$ & 124 & 5.790 & 0.85 \\
\hline 20 & $\mathrm{Ca}$ & 44 & 2.086 & 4.68 & 37 & $\mathrm{Rb}$ & 85 & 70.875 & 2.24 & 51 & $\mathrm{Sb}$ & 121 & 57.210 & 0.80 \\
\hline 20 & $\mathrm{Ca}$ & 46 & 0.004 & 1.97 & 37 & $\mathrm{Rb}^{(*)}$ & 87 & 29.125 & 1.86 & 51 & $\mathrm{Sb}$ & 123 & 42.790 & 0.67 \\
\hline 20 & $\mathrm{Ca}$ & 48 & 0.187 & 3.64 & 38 & $\mathrm{Sr}$ & 84 & 0.563 & 0.64 & 52 & $\mathrm{Te}$ & 120 & 0.090 & -0.85 \\
\hline 21 & $\mathrm{Sc}$ & 45 & 100.000 & 3.20 & 38 & $\mathrm{Sr}$ & 86 & 9.916 & 1.89 & 52 & $\mathrm{Te}$ & 122 & 2.550 & 0.60 \\
\hline 22 & $\mathrm{Ti}$ & 46 & 8.250 & 3.95 & 38 & $\mathrm{Sr}$ & 87 & 6.472 & 1.70 & 52 & $\mathrm{Te}$ & 123 & 0.890 & 0.14 \\
\hline 22 & $\mathrm{Ti}$ & 47 & 7.440 & 3.91 & 38 & $\mathrm{Sr}$ & 88 & 83.049 & 2.81 & 52 & $\mathrm{Te}$ & 124 & 4.740 & 0.87 \\
\hline 22 & $\mathrm{Ti}$ & 48 & 73.720 & 4.90 & 39 & $\mathrm{Y}$ & 89 & 100.000 & 2.27 & 52 & $\mathrm{Te}$ & 125 & 7.070 & 1.04 \\
\hline 22 & $\mathrm{Ti}$ & 49 & 5.410 & 3.77 & 40 & $\mathrm{Zr}$ & 90 & 51.450 & 2.37 & 52 & $\mathrm{Te}$ & 126 & 18.840 & 1.47 \\
\hline 22 & $\mathrm{Ti}$ & 50 & 5.180 & 3.75 & 40 & $\mathrm{Zr}$ & 91 & 11.220 & 1.70 & 52 & $\mathrm{Te}$ & 128 & 31.740 & 1.70 \\
\hline 23 & V & 50 & 0.250 & 1.36 & 40 & $\mathrm{Zr}$ & 92 & 17.150 & 1.89 & 52 & $\mathrm{Te}$ & 130 & 34.080 & 1.73 \\
\hline \multirow[t]{2}{*}{23} & $\mathrm{~V}$ & 51 & 99.750 & 3.96 & 40 & $\mathrm{Zr}$ & 94 & 17.380 & 1.89 & 53 & I & 127 & 100.000 & 1.55 \\
\hline & & & & & 40 & $\mathrm{Zr}$ & 96 & 2.800 & 1.10 & & & & & \\
\hline 54 & $\mathrm{Xe}$ & 124 & 0.127 & -0.61 & 64 & $\mathrm{Gd}$ & 152 & 0.200 & -1.55 & 74 & W & 180 & 0.120 & -2.07 \\
\hline 54 & $\mathrm{Xe}$ & 126 & 0.113 & -0.66 & 64 & $\mathrm{Gd}$ & 154 & 2.180 & -0.52 & 74 & W & 182 & 26.500 & 0.28 \\
\hline 54 & $\mathrm{Xe}$ & 128 & 2.216 & 0.63 & 64 & $\mathrm{Gd}$ & 155 & 14.800 & 0.31 & 74 & W & 183 & 14.310 & 0.01 \\
\hline 54 & $\mathrm{Xe}$ & 129 & 27.438 & 1.72 & 64 & $\mathrm{Gd}$ & 156 & 20.470 & 0.46 & 74 & W & 184 & 30.640 & 0.34 \\
\hline 54 & $\mathrm{Xe}$ & 130 & 4.346 & 0.92 & 64 & $\mathrm{Gd}$ & 157 & 15.650 & 0.34 & 74 & W & 186 & 28.430 & 0.31 \\
\hline 54 & $\mathrm{Xe}$ & 131 & 21.771 & 1.62 & 64 & $\mathrm{Gd}$ & 158 & 24.840 & 0.54 & 75 & $\operatorname{Re}$ & 185 & 35.707 & -0.08 \\
\hline 54 & $\mathrm{Xe}$ & 132 & 26.347 & 1.70 & 64 & $\mathrm{Gd}$ & 160 & 21.860 & 0.48 & 75 & $\operatorname{Re}^{(*)}$ & 187 & 64.293 & 0.18 \\
\hline 54 & $\mathrm{Xe}$ & 134 & 9.743 & 1.27 & 65 & $\mathrm{~Tb}$ & 159 & 100.000 & 0.37 & 76 & Os & 184 & 0.020 & -2.28 \\
\hline 54 & $\mathrm{Xe}$ & 136 & 7.900 & 1.18 & 66 & Dy & 156 & 0.056 & -2.09 & 76 & Os & 186 & 1.597 & -0.38 \\
\hline
\end{tabular}


Table B.1. continued.

\begin{tabular}{|c|c|c|c|c|c|c|c|c|c|c|c|c|c|c|}
\hline Z & Elem. & A & fraction $\%$ & $\log \epsilon$ & $\mathrm{Z}$ & Elem. & $\mathrm{A}$ & fraction $\%$ & $\log \epsilon$ & $\mathrm{Z}$ & Elem. & A & fraction $\%$ & $\log \epsilon$ \\
\hline 55 & Cs & 133 & 100.000 & 1.09 & 66 & Dy & 158 & 0.095 & -1.86 & 76 & Os & 187 & 1.559 & -0.40 \\
\hline 56 & $\mathrm{Ba}$ & 130 & 0.110 & -0.62 & 66 & Dy & 160 & 2.329 & -0.47 & 76 & Os & 188 & 13.294 & 0.54 \\
\hline 56 & $\mathrm{Ba}$ & 132 & 0.100 & -0.67 & 66 & Dy & 161 & 18.889 & 0.44 & 76 & Os & 189 & 16.216 & 0.62 \\
\hline 56 & $\mathrm{Ba}$ & 134 & 2.420 & 0.72 & 66 & Dy & 162 & 25.475 & 0.57 & 76 & Os & 190 & 26.368 & 0.83 \\
\hline 56 & $\mathrm{Ba}$ & 135 & 6.590 & 1.15 & 66 & Dy & 163 & 24.896 & 0.56 & 76 & Os & 192 & 40.947 & 1.02 \\
\hline 56 & $\mathrm{Ba}$ & 136 & 7.850 & 1.23 & 66 & Dy & 164 & 28.260 & 0.62 & 77 & Ir & 191 & 37.230 & 0.97 \\
\hline 56 & $\mathrm{Ba}$ & 137 & 11.230 & 1.38 & 67 & Ho & 165 & 100.000 & 0.54 & 77 & Ir & 193 & 62.770 & 1.19 \\
\hline 56 & $\mathrm{Ba}$ & 138 & 71.700 & 2.19 & 68 & Er & 162 & 0.139 & -1.86 & 78 & $\mathrm{Pt}^{(*)}$ & 190 & 0.012 & -2.25 \\
\hline 57 & $\mathrm{La}^{(*)}$ & 138 & 0.092 & -1.86 & 68 & Er & 164 & 1.601 & -0.80 & 78 & $\mathrm{Pt}$ & 192 & 0.782 & -0.43 \\
\hline 57 & $\mathrm{La}$ & 139 & 99.908 & 1.17 & 68 & Er & 166 & 33.503 & 0.52 & 78 & $\mathrm{Pt}$ & 194 & 32.864 & 1.19 \\
\hline 58 & $\mathrm{Ce}$ & 136 & 0.185 & -1.09 & 68 & Er & 167 & 22.869 & 0.35 & 78 & $\mathrm{Pt}$ & 195 & 33.775 & 1.20 \\
\hline 58 & $\mathrm{Ce}$ & 138 & 0.251 & -0.96 & 68 & Er & 168 & 26.978 & 0.43 & 78 & $\mathrm{Pt}$ & 196 & 25.211 & 1.07 \\
\hline 58 & $\mathrm{Ce}$ & 140 & 88.450 & 1.59 & 68 & Er & 170 & 14.910 & 0.17 & 78 & $\mathrm{Pt}$ & 198 & 7.356 & 0.54 \\
\hline 58 & $\mathrm{Ce}$ & 142 & 11.114 & 0.69 & 69 & Tm & 169 & 100.000 & 0.16 & 79 & $\mathrm{Au}$ & 197 & 100.000 & 0.97 \\
\hline 59 & $\operatorname{Pr}$ & 141 & 100.000 & 0.81 & 70 & $\mathrm{Yb}$ & 168 & 0.126 & -1.99 & 80 & $\mathrm{Hg}$ & 196 & 0.150 & -1.67 \\
\hline 60 & $\mathrm{Nd}$ & 142 & 27.152 & 0.92 & 70 & $\mathrm{Yb}$ & 170 & 3.023 & -0.61 & 80 & $\mathrm{Hg}$ & 198 & 10.040 & 0.16 \\
\hline 60 & $\mathrm{Nd}$ & 143 & 12.174 & 0.57 & 70 & $\mathrm{Yb}$ & 171 & 14.216 & 0.07 & 80 & $\mathrm{Hg}$ & 199 & 16.940 & 0.38 \\
\hline 60 & $\mathrm{Nd}$ & 144 & 23.798 & 0.86 & 70 & $\mathrm{Yb}$ & 172 & 21.754 & 0.25 & 80 & $\mathrm{Hg}$ & 200 & 23.140 & 0.52 \\
\hline 60 & $\mathrm{Nd}$ & 145 & 8.293 & 0.40 & 70 & $\mathrm{Yb}$ & 173 & 16.098 & 0.12 & 80 & $\mathrm{Hg}$ & 201 & 13.170 & 0.28 \\
\hline 60 & $\mathrm{Nd}$ & 146 & 17.189 & 0.72 & 70 & $\mathrm{Yb}$ & 174 & 31.896 & 0.42 & 80 & $\mathrm{Hg}$ & 202 & 29.740 & 0.63 \\
\hline 60 & $\mathrm{Nd}$ & 148 & 5.756 & 0.24 & 70 & $\mathrm{Yb}$ & 176 & 12.887 & 0.02 & 80 & $\mathrm{Hg}$ & 204 & 6.820 & -0.01 \\
\hline 60 & $\mathrm{Nd}$ & 150 & 5.638 & 0.24 & 71 & $\mathrm{Lu}$ & 175 & 97.180 & 0.15 & 81 & $\mathrm{Tl}$ & 203 & 29.515 & 0.45 \\
\hline 62 & $\mathrm{Sm}$ & 144 & 3.066 & -0.50 & 71 & $\mathrm{Lu}^{(*)}$ & 176 & 2.820 & -1.38 & 81 & $\mathrm{Tl}$ & 205 & 70.485 & 0.83 \\
\hline 62 & $\mathrm{Sm}^{(*)}$ & 147 & 15.384 & 0.20 & 72 & $\mathrm{Hf}$ & 174 & 0.161 & -1.88 & 82 & $\mathrm{~Pb}$ & 204 & 1.400 & 0.16 \\
\hline 62 & $\mathrm{Sm}$ & 148 & 11.199 & 0.07 & 72 & $\mathrm{Hf}$ & 176 & 5.240 & -0.37 & 82 & $\mathrm{~Pb}$ & 206 & 24.100 & 1.40 \\
\hline 62 & $\mathrm{Sm}$ & 149 & 13.758 & 0.15 & 72 & $\mathrm{Hf}$ & 177 & 18.580 & 0.18 & 82 & $\mathrm{~Pb}$ & 207 & 22.100 & 1.36 \\
\hline 62 & $\mathrm{Sm}$ & 150 & 7.337 & -0.12 & 72 & $\mathrm{Hf}$ & 178 & 27.280 & 0.35 & 82 & $\mathrm{~Pb}$ & 208 & 52.400 & 1.73 \\
\hline 62 & $\mathrm{Sm}$ & 152 & 26.619 & 0.44 & 72 & $\mathrm{Hf}$ & 179 & 13.630 & 0.05 & 83 & $\mathrm{Bi}$ & 209 & 100.000 & 0.65 \\
\hline 62 & $\mathrm{Sm}$ & 154 & 22.637 & 0.37 & 72 & $\mathrm{Hf}$ & 180 & 35.120 & 0.46 & 90 & $\operatorname{Th}^{(*)}$ & 232 & 100.000 & 0.19 \\
\hline 63 & $\mathrm{Eu}$ & 151 & 47.810 & 0.26 & 73 & $\mathrm{Ta}$ & 180 & 0.012 & -4.00 & 92 & $\mathrm{U}^{(*)}$ & 234 & 0.002 & -4.73 \\
\hline \multirow[t]{2}{*}{63} & $\mathrm{Eu}$ & 153 & 52.190 & 0.30 & 73 & $\mathrm{Ta}$ & 181 & 99.988 & -0.07 & 92 & $\mathrm{U}^{(*)}$ & 235 & 24.167 & -0.65 \\
\hline & & & & & & & & & & 92 & $\mathrm{U}^{(*)}$ & 238 & 75.831 & -0.16 \\
\hline
\end{tabular}

Notes. The proto-solar abundances account for atomic diffusion of 0.07 dex for He and 0.064 dex for all heavier elements as predicted by Vinyoles et al. (2017), as well as radio-active decay. The elements for which no solar abundance is available are based on CI chondrites adjusted for the trend in condensation temperature between the Sun and meteorities (Sect. 3.3). ${ }^{(*)}$ Long-lived radio-active element. 